\title{
Reactivation of Neural Ensembles During Memory Retrieval
}

\author{
Kaycie Kuss Tayler \\ New Orleans, Louisiana
}

M.A., University of Virginia, 2012

B.S. and B.A. University of Utah, 2006

A Dissertation Presented to the Graduate Faculty of the University of Virginia in Candidacy for the Degree of Doctor of Philosophy

Department of Psychology

University of Virginia

May, 2014 


\begin{abstract}
Memory retrieval is thought to involve the recreation of neural activity patterns that were present during learning. This idea stems from Hebbian models of plasticity, which assume that learning-induced changes in synaptic strength increase the probability of neural reactivation during retrieval. Although neural reactivation has long served as the basis of current models of memory it has been extremely difficult to validate experimentally. While it has been possible to follow the activity of individual neurons shortly after learning it has not been possible to examine their activity days and weeks later during retrieval. We addressed this issue by using a stable form of GFP (H2B-GFP) to permanently tag neurons that are active during contextual fear conditioning. We demonstrate that neurons in the hippocampus and neocortex reactivate during the retrieval of context fear (Chapter 2). We next show that patterns of retrieval-driven reactivation shift over time in the hippocampus and amygdala, while remaining stable in the cortex (Chapter 3). Finally, we demonstrate that although the hippocampus is required for neocortical reactivation several days after learning, cortical regions can compensate when this region is inactivated following a weeks-long consolidation period (Chapter 4). Together, the results presented in this dissertation increase our understanding of the hippocampus' role in retrieval-driven reactivation and inform current debates over the accuracy of memory storage and retrieval models.
\end{abstract}




\section{TABLE OF CONTENTS}

ABSTRACT 2

DEDICATION AND ACKNOWLEDGEMENTS 4

CHAPTER 1

General Introduction

CHAPTER 2

Reactivation of Neurons During Recent Memory Retrieval

CHAPTER 3

Reactivation of Neurons During Remote Memory Retrieval

CHAPTER 4 82

Involvement of the Hippocampus in Neocortical Reactivation

CHAPTER 5

130

General Discussion 


\section{DEDICATION}

This dissertation is dedicated to my grandparents, Willie and Joyce Diez.

\section{ACKNOWLEDGEMENTS}

To my committee: Thank you for your input and comments to this body of research. I greatly appreciate your time, thoughtfulness, and support.

To my advisor, Dr. Brian Wiltgen: Thank you for "taking a chance on me" five years ago. I will always be grateful for your intellectual guidance, mentorship, and sense of humor. Just for you: Pearl Jam rocks.

To my advisor, Dr. David Hill: Thank you for trusting me to finish my experiments and dissertation in Dr. Wiltgen's absence. I am truly grateful for the chance I have had to complete my Ph.D. at UVa.

To my co-advisor, Dr. Alev Erisir: Thank you for five years of pedagogical mentorship, professional advising, and friendship. Your optimism and example made me believe that all of this was possible.

To Amy O'Coin and the UVa Gilmer Vivarium Staff, Rosa and McKennon: Thank you for helping me keep the 175 experimental area quiet, supplied with emergency power, and free of construction many times. Thank you also for help with the colony 
management and doxycycline chow. This work could not have happened without you.

To Rachel Bailey: Thank you for reminding me of how far I have come and what I am capable of. Thank you for not letting me give-up when things became difficult. You are a true friend.

To my parents, Brad and Kathy Kuss: There really are not words to express how grateful I am to you. I try to honor you each day in my personal and professional life. Geaux Tigers.

To Him: "Hand in hand we come, Leif and I, to lay this book in your lap. Say you're surprised? Say you like it? Say it's just what you wanted? Because it's yoursbecause we love you." 
Chapter 1

General Introduction 
Traditional models of learning and memory assume that hippocampal and neocortical circuits reactivate during the retrieval of episodic and contextual memories (Eichenbaum, 2004; Frankland \& Bontempi, 2005; Morris et al., 2003). Consistent with a reactivation hypothesis, many brain regions that are active during learning are also engaged during retrieval (Frankland et al., 2004; Hall, Thomas, \& Everitt, 2001; Lonergan et al., 2010; Schacter et al., 1999). Although reactivation has served as the foundation of memory models for several decades, it has not been possible to determine if the same neurons that encode memory reactivate during retrieval. Using a novel transgenic animal, the H2BGFP TetTag mouse, this dissertation examines for the first time whether neurons in the hippocampus and neocortex reactivate during context fear memory retrieval (Chapter 2). We next examine whether patterns of reactivation shift over time during the retrieval of remote contextual memory (Chapter 3). Finally, in Chapter 4 we examine whether the hippocampus is required for neocortical reactivation at recent and remote time points.

\section{The Standard Model of Systems Consolidation}

Cortical reactivation during memory retrieval is thought to initially require the hippocampus, but occur independently with the passage of time (Alvarez \& Squire, 1994). The process by which memories become independent of the hippocampus and are stored in the neocortex is called systems consolidation (Frankland \& Bontempi, 2005; Squire, 1992; Squire \& Bayley, 2007; Wiltgen, Brown, Talton, \& Silva, 2004). This concept is based on the finding that hippocampus damage impairs recently acquired episodic memories but does not affect those formed in the distant past (Bayley, Hopkins, \& Squire, 2003; Kapur \& Brooks, 1999; Kirwan, Bayley, Galván, \& Squire, 2008; 
Rempel-Clower, Zola, Squire, \& Amaral, 1996; Zola-Morgan, Squire, \& Amaral, 1986) but see (Moscovitch et al., 2005; Winocur \& Moscovitch, 2011). For example, patient E.P. (bilateral hippocampus damage) could remember the city where he was raised fifty years prior, but was unable to learn about a new neighborhood where he had lived for six years (Teng \& Squire, 1999). To explain these findings, it was proposed that memory retrieval initially requires the hippocampus because this structure is able to reactivate neocortical regions that were engaged during learning. Over time, continued reactivation strengthens the connections within cortical networks until memory can eventually be retrieved without the support of the hippocampus (Alvarez \& Squire, 1994; Squire \& Wixted, 2011). Consistent with this idea, damage to the neocortex (e.g. lateral temporal, frontal) leads to impairments in remote memory (Bayley, Gold, Hopkins, \& Squire, 2005; Bayley et al., 2003; Mangels, Gershberg, Shimamura, \& Knight, 1996; Murre, Graham, \& Hodges, 2001; Reed \& Squire, 1998; Squire \& Bayley, 2007; Squire \& Wixted, 2011).

\section{Animal Models of Systems Consolidation}

Systems consolidation has been observed in animals using a number of behavioral procedures (e.g. context fear conditioning, spatial learning, social transmission of food preferences, trace eyeblink conditioning). In these tasks, inactivation of the hippocampus impairs memory retrieval shortly after learning, but has no effect when performed weeks or months later (Anagnostaras, Maren, \& Fanselow, 1999; Clark, Broadbent, Zola, \& Squire, 2002; Kim \& Fanselow, 1992; Lesburgueres et al., 2011; Maviel, Durkin, Menzaghi, \& Bontempi, 2004; Ross \& Eichenbaum, 2006; Takehara, Kawahara, \& Kirino, 2003; Takehara, Kawahara, Takatsuki, \& Kirino, 2002; Tayler, Tanaka, Reijmers, 
\& Wiltgen, 2013) but see (Clark, Broadbent, \& Squire, 2005; Sutherland, Sparks, \& Lehmann, 2010). In addition, several regions of the neocortex become more important for memory retrieval with the passage of time. Remote memory for context fear, spatial locations and eyeblink conditioning requires the medial prefrontal cortex (Frankland, Bontempi, Talton, Kaczmarek, \& Silva, 2004; Maviel et al., 2004; Takehara et al., 2003; Takehara-Nishiuchi, Maal-Bared, \& Morrissey, 2011; Takehara-Nishiuchi \& McNaughton, 2008), while socially transmitted food preferences depend on the oribitofrontal cortex (Lesburgueres et al., 2011). Increased immediate early gene expression in the neocortex is also observed during remote memory retrieval in these tasks (Lesburgueres et al., 2011; Maviel et al., 2004). As a result, these animal models have been used extensively to examine the neurobiological mechanisms of systems consolidation.

\section{Assumptions of SMC}

A number of predictions made by SMC have been confirmed experimentally. Electrophysiological studies demonstrate that hippocampal and neocortical circuits are reactivated after learning during periods of sleep and inactivity (Hoffman \& McNaughton, 2002; Louie \& Wilson, 2001; Nadasdy, Hirase, Czurko, Csicsvari, \& Buzsaki, 1999; Qin, McNaughton, Skaggs, \& Barnes, 1997; Siapas \& Wilson, 1998; Wilson \& McNaughton, 1994). Reactivation during these periods is initiated by sharp wave-ripple complexes (SPW-R) in the CA3 region of the hippocampus (Buzsaki, 1989; Chrobak \& Buzsaki, 1996; Sullivan et al., 2011). Blocking SPW-R events or disrupting the fibers by which they are transmitted to the neocortex impairs the consolidation of 
recently acquired memories (Daumas, Halley, Frances, \& Lassalle, 2005; Girardeau, Benchenane, Wiener, Buzsaki, \& Zugaro, 2009; Jadhav, Kemere, German, \& Frank, 2012).

Studies have also shown that neocortical plasticity is required for memory consolidation (Takehara-Nishiuchi, Nakao, Kawahara, Matsuki, \& Kirino, 2006). For example, heterozygous deletion of the $\alpha \mathrm{CaMKII}$ gene impairs plasticity and synaptogenesis in the neocortex without affecting hippocampal function. These changes have no effect on learning but produce severe memory loss weeks later (Frankland, O'Brien, Ohno, Kirkwood, \& Silva, 2001). Consistent with this finding, spine growth in the anterior cingulate and orbitofrontal cortex is necessary for remote memory formation (Lesburgueres et al., 2011; Vetere et al., 2011). Together, these studies indicate that the consolidation of recently formed memories depends on the ability of the neocortex to form new synaptic connections (Frankland \& Bontempi, 2005). Theta-entrained activity in the hippocampus and neocortex may allow plastic changes to occur that mediate information storage across these networks (Siapas, Lubenov, \& Wilson, 2005).

Although SMC has been enormously useful, a number of its core assumptions remain untested (Alvarez \& Squire, 1994). For example, it has not been possible to determine if the same neocortical neurons that were engaged during learning reactivate weeks later during memory retrieval. It has also been impossible to inactivate hippocampal neurons that were active during learning to determine if this structure drives the reactivation of neocortical networks. However, recent advances in transgenic neural tagging now allow 
researchers to examine assumptions that have been central to theories of memory consolidation for decades (Alvarez \& Squire, 1994; Marr, 1971; McClelland, McNaughton, \& O'Reilly, 1995). These include:

Assumption 1: the same neurons that encode memory in the hippocampus and neocortex are reactivated during retrieval.

Assumption 2: early after learning, the hippocampus drives reactivation of neocortical ensembles during memory retrieval.

Assumption 3: as time passes, neocortical ensembles are reactivated during memory retrieval without input from the hippocampus.

\section{Identifying Reactivated Neurons}

Immediate early gene (IEG) expression is commonly used to identify activated neurons during learning or retrieval. Expression of these genes is regulated by excitatory synaptic transmission and, in the hippocampus, is closely related to place cell activity (Guzowski, 2002; Guzowski, McNaughton, Barnes, \& Worley, 1999; Vazdarjanova et al., 2006). IEGs can also be used to examine reactivation of individual neurons during two separate events. For example, Guzowski and colleagues developed a technique called cellular compartment analysis of temporal activity by fluorescence in situ hybridization (catFISH) that utilized the time course of IEG mRNA transcription to identify reactivated neurons (Guzowski et al., 2005). They found that a large population of hippocampal neurons was reactivated when rats explored the same spatial environment twice within a twentyminute period (Guzowski et al., 1999). Reactivation was significantly reduced when animals explored two different environments. While extremely useful, this technique is 
limited by the rapid degradation of mRNA. As a result, reactivated neurons can only be identified when sessions occur in close proximity $(\approx 20-30$ minutes $)$.

Reijmers et al. (2007) circumvented the temporal limitations of catFISH by using transgenic mice to label activated neurons. In their mice, the c-Fos promoter was used to drive expression of tetracycline transactivator protein (tTA) during learning. tTA expression shows robust overlap with endogenous c-Fos in this system (Liu et al., 2012). Activation of the tetO promoter by tTA led to the expression of tau-LacZ, which could be used to identify activated neurons. Activation of the tetO promoter also produced the expression of a doxycycline-insensitive form of tTA, which sustained tau-LacZ activity for several days. As a result, neurons that were labeled during learning could later be identified during testing. During memory retrieval, expression of Zif-268 was used as an indicator of activity. Neurons that were activated during learning and retrieval were double positive for tau-LacZ and Zif-268.

Using a Pavlovian fear conditioning procedure, the authors were able to observe reactivation of individual amygdala neurons when memory was retrieved three days after training. The extent of reactivation in the basolateral nucleus of the amygdala (BLA) correlated with memory strength for context fear, while reactivation in the lateral nucleus of the amygdala (LA) correlated with memory strength for tone fear. Reactivation was significantly lower in mice that did not retrieve memory (fear conditioned-no retrieval group) and in homecage control animals that were not trained or tested. Together, these data demonstrate that neural reactivation in the BLA is important to fear memory 
retrieval.

\section{Testing SMC Assumptions using H2B-GFP TetTag Mice}

By using similar methods of transgenic neural tagging, we can now address the first untested assumption of SMC: that neural reactivation occurs within the hippocampus and neocortex during memory retrieval. We have chosen to examine reactivation during the retrieval of context fear conditioning using a transgenic mouse that expresses a longlasting, activity-dependent form of GFP (H2B-GFP TetTag) (Tayler et al., 2011; Tayler et al., 2013). In these mice, GFP is fused with the human histone protein HIST1H2BJ (Kanda, Sullivan, \& Wahl, 1998). The resulting protein (H2B-GFP) is localized to the nucleus and remains stable for several weeks after training. To restrict tagging to neurons active during learning, tetO-H2BGFP mice were bred with the fos-tTA mice described above. c-Fos expression was used to identify neurons active during context testing. We anticipated that mice retrieving a memory for the training context would demonstrate reactivation of hippocampal and neocortical neurons. If reactivation occurred, we could then test the second and third assumptions of SMC; we could determine if reactivation of neocortical neurons requires the hippocampus at recent, but not remote time points. To do this, we trained mice using contextual fear conditioning to tag hippocampal and cortical neurons with H2B-GFP during learning. We then inactivated the hippocampus prior to memory retrieval 2 or 14 days later and examined whether neocortical neurons reactivate during retrieval. Based on SMC, we anticipated that hippocampal inactivation would impair neocortical reactivation at recent, but not remote time points. 
It is important to note that a growing literature indicates the hippocampus sometimes plays a prolonged role in memory retrieval (Moscovitch et al., 2005; Sutherland et al., 2010; Wiltgen et al., 2010; Winocur \& Moscovitch, 2011). In context fear conditioning, for example, hippocampus lesions can impair both recent and remote memory (Lehmann, Lacanilao, \& Sutherland, 2007). Whether or not systems consolidation occurs appears to depend on the quality of memory that is retrieved during testing and the ability of neocortical regions to compensate when the hippocampus is compromised (Goshen et al., 2011; Wiltgen \& Tanaka, 2013; Wiltgen et al., 2010; Winocur, Frankland, Sekeres, Fogel, \& Moscovitch, 2009; Winocur, Moscovitch, \& Sekeres, 2007). A recent study suggests that this is the case (Goshen et al., 2011). When the hippocampus was inactivated 28 days after context fear conditioning, the anterior cingulate cortex showed an increase in activity and became essential for memory retrieval. When cortical compensation was prevented (via rapid optogenetic silencing) remote memory retrieval was dependent on the hippocampus. These results suggest that remote memories can be retrieved by either the hippocampus or the neocortex. Which system is used appears to depend on a number of factors including the passage of time, the presence of reminder cues and the proliferation of new neurons (Alvares Lde et al., 2012; Kitamura et al., 2009; Winocur et al., 2009).

In this dissertation, Chapter 2 determines whether hippocampal and neocortical neurons engaged during learning reactivate during recent memory retrieval. We hypothesized that reactivation would occur in mice trained and tested two days later in the same environment, but not in mice trained and tested in different environments. Chapter 3 then 
examines whether hippocampus and neocortex reactivation patterns change over time. Based on the SMC, we predicted that remote memory retrieval would reactivate the neocortex but not the hippocampus. Finally, Chapter 4 explores whether the hippocampus is necessary for neocortical reactivation at recent and remote time points. Based on the SMC, we hypothesized that hippocampus inactivation would impair the ability of neocortical neurons to reactivate when the inactivation is performed two days, but not fourteen days, after training. Together, these chapters demonstrate the first direct tests of reactivation assumptions held by the SMC and work to improve the accuracy of traditional models of learning and memory. 


\section{REFERENCES}

Alvares Lde, O., Einarsson, E. O., Santana, F., Crestani, A. P., Haubrich, J., Cassini, L. F., . . Quillfeldt, J. A. (2012). Periodically reactivated context memory retains its precision and dependence on the hippocampus. Hippocampus, 22(5), 1092-1095.

Alvarez, P., \& Squire, L. R. (1994). Memory consolidation and the medial temporal lobe: a simple network model. Proc Natl Acad Sci US A, 91(15), 7041-7045.

Anagnostaras, S. G., Maren, S., \& Fanselow, M. S. (1999). Temporally graded retrograde amnesia of contextual fear after hippocampal damage in rats: within-subjects examination. J Neurosci, 19(3), 1106-1114.

Bayley, P. J., Gold, J. J., Hopkins, R. O., \& Squire, L. R. (2005). The neuroanatomy of remote memory. Neuron, 46(5), 799-810.

Bayley, P. J., Hopkins, R. O., \& Squire, L. R. (2003). Successful Recollection of Remote Autobiographical Memories by Amnesic Patients with Medial Temporal Lobe Lesions. Neuron, 38(1), 135-144.

Buzsaki, G. (1989). Two-stage model of memory trace formation: a role for "noisy" brain states. Neuroscience, 31(3), 551-570.

Chrobak, J. J., \& Buzsaki, G. (1996). High-frequency oscillations in the output networks of the hippocampal-entorhinal axis of the freely behaving rat. $J$ Neurosci, 16(9), 3056-3066.

Clark, R. E., Broadbent, N. J., \& Squire, L. R. (2005). Hippocampus and remote spatial memory in rats. Hippocampus, 15(2), 260-272. 
Clark, R. E., Broadbent, N. J., Zola, S. M., \& Squire, L. R. (2002). Anterograde amnesia and temporally graded retrograde amnesia for a nonspatial memory task after lesions of hippocampus and subiculum. J Neurosci, 22(11), 4663-4669.

Daumas, S., Halley, H., Frances, B., \& Lassalle, J. M. (2005). Encoding, consolidation, and retrieval of contextual memory: differential involvement of dorsal CA3 and CA1 hippocampal subregions. Learning \& memory, 12(4), 375-382.

Eichenbaum, H. (2004). Hippocampus: cognitive processes and neural representations that underlie declarative memory. Neuron, 44(1), 109-120.

Frankland, P. W., \& Bontempi, B. (2005). The organization of recent and remote memories. Nat Rev Neurosci, 6(2), 119-130.

Frankland, P. W., Bontempi, B., Talton, L. E., Kaczmarek, L., \& Silva, A. J. (2004). The involvement of the anterior cingulate cortex in remote contextual fear memory. Science, 304(5672), 881-883.

Frankland, P. W., O'Brien, C., Ohno, M., Kirkwood, A., \& Silva, A. J. (2001). AlphaCaMKII-dependent plasticity in the cortex is required for permanent memory. Nature, 411(6835), 309-313.

Girardeau, G., Benchenane, K., Wiener, S. I., Buzsaki, G., \& Zugaro, M. B. (2009). Selective suppression of hippocampal ripples impairs spatial memory. Nature neuroscience, 12(10), 1222-1223.

Goshen, I., Brodsky, M., Prakash, R., Wallace, J., Gradinaru, V., Ramakrishnan, C., \& Deisseroth, K. (2011). Dynamics of Retrieval Strategies for Remote Memories. Cell, 147(3), 678-689. 
Guzowski, J. F. (2002). Insights into immediate-early gene function in hippocampal memory consolidation using antisense oligonucleotide and fluorescent imaging approaches. Hippocampus, 12(1), 86-104.

Guzowski, J. F., McNaughton, B. L., Barnes, C. A., \& Worley, P. F. (1999). Environment-specific expression of the immediate-early gene Arc in hippocampal neuronal ensembles. Nat Neurosci, 2(12), 1120-1124.

Guzowski, J. F., Timlin, J. A., Roysam, B., McNaughton, B. L., Worley, P. F., \& Barnes, C. A. (2005). Mapping behaviorally relevant neural circuits with immediate-early gene expression. Current Opinion in Neurobiology, 15(5), 599-606.

Hall, J., Thomas, K. L., \& Everitt, B. J. (2001). Cellular imaging of zif268 expression in the hippocampus and amygdala during contextual and cued fear memory retrieval: selective activation of hippocampal CA1 neurons during the recall of contextual memories. J Neurosci, 21(6), 2186-2193.

Hoffman, K. L., \& McNaughton, B. L. (2002). Coordinated reactivation of distributed memory traces in primate neocortex. Science, 297(5589), 2070-2073.

Jadhav, S. P., Kemere, C., German, P. W., \& Frank, L. M. (2012). Awake Hippocampal Sharp-Wave Ripples Support Spatial Memory. Science, 336(6087), 1454-1458.

Kanda, T., Sullivan, K. F., \& Wahl, G. M. (1998). Histone-GFP fusion protein enables sensitive analysis of chromosome dynamics in living mammalian cells. Current Biology, 8(7), 377-385.

Kapur, N., \& Brooks, D. J. (1999). Temporally-specific retrograde amnesia in two cases of discrete bilateral hippocampal pathology. Hippocampus, 9(3), 247-254. 
Kim, J. J., \& Fanselow, M. S. (1992). Modality-specific retrograde amnesia of fear. Science, 256(5057), 675-677.

Kirwan, C. B., Bayley, P. J., Galván, V. V., \& Squire, L. R. (2008). Detailed recollection of remote autobiographical memory after damage to the medial temporal lobe. Proceedings of the National Academy of Sciences, 105(7), 2676-2680.

Kitamura, T., Saitoh, Y., Takashima, N., Murayama, A., Niibori, Y., Ageta, H., .. . Inokuchi, K. (2009). Adult neurogenesis modulates the hippocampus-dependent period of associative fear memory. Cell, 139(4), 814-827.

Lehmann, H., Lacanilao, S., \& Sutherland, R. J. (2007). Complete or partial hippocampal damage produces equivalent retrograde amnesia for remote contextual fear memories. Eur J Neurosci, 25(5), 1278-1286.

Lesburgueres, E., Gobbo, O. L., Alaux-Cantin, S., Hambucken, A., Trifilieff, P., \& Bontempi, B. (2011). Early tagging of cortical networks is required for the formation of enduring associative memory. Science, 331, 924-928.

Liu, X., Ramirez, S., Pang, P. T., Puryear, C. B., Govindarajan, A. F., Deisseroth, K., \& Tonegawa, S. (2012). Optogenetic stimulation of a hippocampal engram activates fear memory recall. Nature, 484(19 April), 381-387.

Lonergan, M. E., Gafford, G. M., Jarome, T. J., \& Helmstetter, F. J. (2010). Timedependent expression of Arc and zif268 after acquisition of fear conditioning. Neural plasticity, 2010, 139891.

Louie, K., \& Wilson, M. A. (2001). Temporally structured replay of awake hippocampal ensemble activity during rapid eye movement sleep. Neuron, 29(1), 145-156. 
Mangels, J. A., Gershberg, F. B., Shimamura, A. O., \& Knight, R. T. (1996). Impaired Retrieval From Remote Memory in Patients With Frontal Lobe Damage. Neuropsychology, 10(1), 32-41.

Marr, D. (1971). Simple memory: a theory for archicortex. Philosophical transactions of the Royal Society of London. Series B, Biological sciences, 262(841), 23-81.

Maviel, T., Durkin, T. P., Menzaghi, F., \& Bontempi, B. (2004). Sites of Neocortical Reorganization Critical for Remote Spatial Memory. Science, 305(5680), 96-99.

McClelland, J. L., McNaughton, B. L., \& O'Reilly, R. C. (1995). Why there are complementary learning systems in the hippocampus and neocortex: insights from the successes and failures of connectionist models of learning and memory. Psychol Rev, 102(3), 419-457.

Morris, R. G., Moser, E. I., Riedel, G., Martin, S. J., Sandin, J., Day, M., \& O'Carroll, C. (2003). Elements of a neurobiological theory of the hippocampus: the role of activity-dependent synaptic plasticity in memory. Philosophical transactions of the Royal Society of London. Series B, Biological sciences, 358(1432), 773-786.

Moscovitch, M., Rosenbaum, R. S., Gilboa, A., Addis, D. R., Westmacott, R., Grady, C., ... Nadel, L. (2005). Functional neuroanatomy of remote episodic, semantic and spatial memory: a unified account based on multiple trace theory. J. Anat., 207, 35-66.

Murre, J. M., Graham, K. S., \& Hodges, J. R. (2001). Semantic dementia: relevance to connectionist models of long-term memory. Brain, 124, 647-675. 
Nadasdy, Z., Hirase, H., Czurko, A., Csicsvari, J., \& Buzsaki, G. (1999). Replay and time compression of recurring spike sequences in the hippocampus. The Journal of Neuroscience, 19(21), 9497-9507.

Qin, Y., McNaughton, B., Skaggs, W., \& Barnes, C. A. (1997). Memory reprocessing in corticocortical and hippocampocortical neuronal ensembles. Philos Trans R Soc Lond B Biol Sci, 352(1360).

Reed, J. M., \& Squire, L. R. (1998). Retrograde Amnesia for Facts and Events: Findings from Four New Cases. J Neurosci, 18(10), 3943-3954.

Reijmers, L. G., Perkins, B. L., Matsuo, N., \& Mayford, M. (2007). Localization of a stable neural correlate of associative memory. Science, 317(5842), 1230-1233.

Rempel-Clower, N. L., Zola, S. M., Squire, L. R., \& Amaral, D. G. (1996). Three cases of enduring memory impairment after bilateral damage limited to the hippocampal formation. $J$ Neurosci, 16(16), 5233-5255.

Ross, R. S., \& Eichenbaum, H. (2006). Dynamics of hippocampal and cortical activation during consolidation of a nonspatial memory. J Neurosci, 26(18), 4852-4859.

Schacter, D. L., Curran, T., Reiman, E. M., Chen, K., Bandy, D. J., \& Frost, J. T. (1999). Medial temporal lobe activation during episodic encoding and retrieval: a PET study. Hippocampus, 9(5), 575-581.

Siapas, A. G., Lubenov, E. V., \& Wilson, M. A. (2005). Prefrontal phase locking to hippocampal theta oscillations. Neuron, 46(1), 141-151.

Siapas, A. G., \& Wilson, M. A. (1998). Coordinated interactions between hippocampal ripples and cortical spindles during slow-wave sleep. Neuron, 21(5), 1123-1128. 
Squire, L. R. (1992). Memory and the hippocampus: a synthesis from findings with rats, monkeys, and humans. Psychol Rev, 99(2), 195-231.

Squire, L. R., \& Bayley, P. J. (2007). The neuroscience of remote memory. Curr Opin Neurobiol, 17(2), 185-196.

Squire, L. R., \& Wixted, J. T. (2011). The cognitive neuroscience of human memory since H.M. Annu Rev Neurosci, 34, 259-288.

Sullivan, D., Csicsvari, J., Mizuseki, K., Montgomery, S., Diba, K., \& Buzsaki, G. (2011). Relationships between hippocampal sharp waves, ripples, and fast gamma oscillation: influence of dentate and entorhinal cortical activity. $J$ Neurosci, $31(23), 8605-8616$.

Sutherland, R. J., Sparks, F. T., \& Lehmann, H. (2010). Hippocampus and retrograde amnesia in the rat model: a modest proposal for the situation of systems consolidation. Neuropsychologia, 48(8), 2357-2369.

Takehara, K., Kawahara, S., \& Kirino, Y. (2003). Time-dependent reorganization of the brain components underlying memory retention in trace eyeblink conditioning. $J$ Neurosci, 23(30), 9897-9905.

Takehara, K., Kawahara, S., Takatsuki, K., \& Kirino, Y. (2002). Time-limited role of the hippocampus in the memory for trace eyeblink conditioning in mice. Brain Res, 951(2), 183-190.

Takehara-Nishiuchi, K., Maal-Bared, G., \& Morrissey, M. D. (2011). Increased Entorhinal-Prefrontal Theta Synchronization Parallels Decreased EntorhinalHippocampal Theta Synchronization during Learning and Consolidation of Associative Memory. Front Behav Neurosci, 5, 90. 
Takehara-Nishiuchi, K., \& McNaughton, B. L. (2008). Spontaneous changes of neocortical code for associative memory during consolidation. Science, 322(5903), 960-963.

Takehara-Nishiuchi, K., Nakao, K., Kawahara, S., Matsuki, N., \& Kirino, Y. (2006). Systems consolidation requires postlearning activation of NMDA receptors in the medial prefrontal cortex in trace eyeblink conditioning. J Neurosci, 26(19), 50495058.

Tayler, K. K., Lowry, E., Tanaka, K., Levy, B., Reijmers, L., Mayford, M., \& Wiltgen, B. J. (2011). Characterization of NMDAR-Independent Learning in the Hippocampus. Front Behav Neurosci, 5, 28.

Tayler, K. K., Tanaka, K. Z., Reijmers, L. G., \& Wiltgen, B. J. (2013). Reactivation of Neural Ensembles during the Retrieval of Recent and Remote Memory. Curr Biol, 23(2), 99-106.

Teng, E., \& Squire, L. R. (1999). Memory for places learned long ago is intact after hippocampal damage. Nature, 400, 675-677.

Vazdarjanova, A., Ramirez-Amaya, V., Insel, N., Plummer, T. K., Rosi, S., Chowdhury, S., ... Barnes, C. A. (2006). Spatial exploration induces ARC, a plasticity-related immediate-early gene, only in calcium/calmodulin-dependent protein kinase IIpositive principal excitatory and inhibitory neurons of the rat forebrain. The Journal of Comparative Neurology, 498(3), 317-329.

Vetere, G., Restivo, L., Cole, C. J., Ross, P. J., Ammassari-Teule, M., Josselyn, S. A., \& Frankland, P. W. (2011). Spine growth in the anterior cingulate cortex is 
necessary for the consolidation of contextual fear memory. Proc Natl Acad Sci U $S A, 108(20), 8456-8460$.

Wilson, M. A., \& McNaughton, B. L. (1994). Reactivation of hippocampal ensemble memories during sleep. Science, 265(5172), 676-679.

Wiltgen, B. J., Brown, R. A., Talton, L. E., \& Silva, A. J. (2004). New circuits for old memories: the role of the neocortex in consolidation. Neuron, 44(1), 101-108.

Wiltgen, B. J., \& Tanaka, K. Z. (2013). Systems consolidation and the content of memory. Neurobiol Learn Mem. doi: 10.1016/j.nlm.2013.06.001

Wiltgen, B. J., Zhou, M., Cai, Y., Balaji, J., Karlsson, M. G., Parivash, S. N., . . Silva, A. J. (2010). The hippocampus plays a selective role in the retrieval of detailed contextual memories. Curr Biol, 20(15), 1336-1344.

Winocur, G., Frankland, P. W., Sekeres, M., Fogel, S., \& Moscovitch, M. (2009). Changes in context-specificity during memory reconsolidation: selective effects of hippocampal lesions. Learn Mem, 16(11), 722-729.

Winocur, G., \& Moscovitch, M. (2011). Memory transformation and systems consolidation. Journal of the International Neuropsychological Society, 17(5), 766-780.

Winocur, G., Moscovitch, M., \& Sekeres, M. (2007). Memory consolidation or transformation: context manipulation and hippocampal representations of memory. Nat Neurosci, 10(5), 555-557.

Zola-Morgan, S., Squire, L. R., \& Amaral, D. G. (1986). Human amnesia and the medial temporal region: enduring memory impairment following a bilateral lesion limited to field CA1 of the hippocampus. J Neurosci, 6(10), 2950-2967. 
Chapter 2

Reactivation of Neurons During Recent Memory Retrieval 


\section{ABSTRACT}

Episodic memories are encoded within hippocampal and neocortical circuits. Retrieving these memories is assumed to involve reactivation of neural ensembles that were engaged during learning. However, this assumption has been difficult to verify experimentally. While it has been possible to follow the activity of individual neurons shortly after learning, it has not been possible to examine their activity days later during retrieval. We addressed this issue by using a stable form of GFP (H2B-GFP) to tag neurons active during contextual fear conditioning. We then examined whether neurons engaged during learning reactivate during subsequent memory retrieval. We hypothesized that reactivation would occur in mice trained and tested two days later in the same environment. We also predicted that reactivation would not occur in mice trained and tested in separate environments or in mice that were trained but not tested.

We observed that H2B-GFP expression was increased by learning and could be regulated using the administration of doxycycline chow (DOX). Using this system, we found a large network of neurons in the hippocampus, amygdala and neocortex that were active during context fear conditioning and subsequent memory retrieval 2 days later. Reactivation was contingent on memory retrieval and was not observed when animals were trained and tested in different environments or when mice were trained but not tested. Reactivation was not observed in a control brain region presumed not to support contextual memory retrieval. In summary, we observed that retrieving a recently formed context fear memory induces widespread reactivation of neurons in the hippocampus, cortex, and amygdala. 


\section{INTRODUCTION}

Episodic and contextual memories can be retrieved months and years after they are formed. Retrieving these memories is assumed to involve reactivation of hippocampal and neocortical networks that were established during learning (Eichenbaum, 2004; Frankland \& Bontempi, 2005; Morris et al., 2003). Consistent with this idea, many brain regions that are active during learning are also engaged during testing (Frankland, Bontempi, Talton, Kaczmarek, \& Silva, 2004; Hall, Thomas, \& Everitt, 2001; Lonergan, Gafford, Jarome, \& Helmstetter, 2010; Schacter et al., 1999). However, it has not been possible to determine if the same neurons that encode memory in these regions are later reactivated during retrieval.

Fluorescence in situ hybridization (FISH) studies using the immediate early genes Arc and Homer indicate that hippocampal and neocortical neurons are reactivated when animals explore the same spatial environment twice within a 30 minute period (Guzowski, McNaughton, Barnes, \& Worley, 1999; Kubik, Miyashita, Kubik-Zahorodna, \& Guzowski, 2012). Longer intervals cannot be examined with this technique, however, because mRNA for these genes rapidly decays. To overcome this issue, we used newly engineered transgenic mice that express a long-lasting, activity dependent form of GFP. In these animals, activation of the c-Fos promoter during learning leads to the expression of human histone H2B-GFP, a fusion protein that takes several weeks to degrade (Foudi et al., 2009; Kanda, Sullivan, \& Wahl, 1998; Tayler et al., 2011; Tumbar et al., 2004). As a result, the activity of neurons labeled with H2B-GFP can be examined days and weeks after learning. A similar strategy was used to demonstrate reactivation of 
amygdala neurons three days after learning in tau-LacZ reporter mice (Reijmers, Perkins, Matsuo, \& Mayford, 2007).

In the current experiments, we examined memory retrieval using a context fear conditioning task that is dependent on the hippocampus, amygdala and neocortex (Frankland \& Bontempi, 2005; Maren, 2001; Wiltgen \& Fanselow, 2003). Neurons were labeled with H2B-GFP during learning and then re-examined two days later during memory retrieval. We found a large network of tagged neurons in the hippocampus, amygdala and neocortex that were reactivated two days after training. These same networks were not reactivated when animals were trained and tested in different environments, or when mice were trained but not tested. These results suggest that memory retrieval involves the reactivation of individual neurons that were engaged during learning. 


\section{METHODS}

\section{Subjects}

The TetTag mice used in these experiments were generated by crossing transgenic mice that express a histone 2B-GFP fusion protein controlled by the tetO promoter (strain Tg(tetO-HIST1H2BJ/GFP) 47Efu/J; stock number 005104; Jackson Laboratory, Bar Harbor, Maine) with mice that express tetracycline-transactivator (tTA) protein under control of the c-Fos promoter (fos-tTA; provided by Mark Mayford) (Tayler et al., 2011). TetTag mice were maintained in a C57BL/6J background. B6/129 F1 hybrids were generated by breeding TetTag mice with 129S6 mice (Taconic, Hudson, NY). Mice were born and raised on DOX chow (40 mg/ $\mathrm{kg}$ ) to prevent H2B-GFP expression prior to experimental manipulations. Mice remained on a 12-h light/dark cycle with ad libitum access to food and water for the duration of the experiment. Experimental manipulations were performed on 8-12 week old male and female mice during the light phase of the cycle. All experiments were approved by the University of Virginia Animal Care and Use Committee.

\section{Apparatus}

Mice were trained in contextual fear conditioning chambers (context A) that were housed in sound-attenuated boxes. The chambers contained a stainless steel grid floor, overhead LED lighting (providing broad spectrum and infrared light) and a scanning CCD video camera (Med Associates Inc., St. Albans, VT). The chamber and drop pan were cleaned with $95 \%$ ethanol before each training session. Contextual fear memory was assessed by placing the mice in context A or a new environment (context B) and measuring the 
freezing response (i.e. percentage of test time spent in an immobile, species-specific defensive posture). Freezing measurements were automated using the VideoFreeze system (Med Associates Inc., St. Albans, VT) (Anagnostaras et al., 2010). In context B, all visible light was turned off, a curved white plastic back wall was inserted, a white plastic insert covered the floor, and Saniwipes (Nice-Pak Products, Inc.) were used to clean the chamber.

\section{Behavioral Procedures}

In the first experiment (Figure 1), TetTag mice in a C57BL/6J background were taken off DOX five days prior to training. During conditioning, mice were allowed to explore context $\mathrm{A}$ for three minutes prior to the onset of 5 footshocks $(0.75 \mathrm{~mA}, 2 \mathrm{~s})$ separated by a 20s intertrial interval (ITI). In all subsequent experiments, TetTag mice on a B6/129 F1 hybrid background were taken off DOX for three days and trained with three shocks $(0.5 \mathrm{~mA}, 2 \mathrm{~s})$ to achieve a similar level of freezing and H2B-GFP expression as observed in C57BL/6J mice. Unsignaled shocks were used because a previous experiment from our lab showed that similar training parameters produced temporally-graded retrograde amnesia (Wiltgen et al., 2010). Following training, all mice were given high concentration DOX chow $(1 \mathrm{~g} / \mathrm{kg})$ for 24 hours to rapidly suppress GFP tagging and remained on $40 \mathrm{mg} / \mathrm{kg}$ DOX chow for the duration of the study. Memory was assessed two days later by returning the mice to the training context (A) or a novel environment (B) for five minutes and measuring the freezing response. An additional No Retrieval group was trained identically off DOX in context A and given high concentration DOX chow, but was sacrificed two days later directly from homecage. 


\section{Immunohistochemistry}

Ninety minutes after behavioral testing, mice were transcardially perfused with $4 \%$ PFA, followed by $24 \mathrm{~h}$ post-fixation in the same solution. Free-floating $40 \mu \mathrm{m}$ coronal sections were prepared using a vibrotome and first blocked for one hour at room temperature in a solution containing $0.1 \%$ BSA, $0.2 \%$ Triton X, and $2 \%$ Normal Goat Serum. Sections were then stained in fresh blocking solution containing anti-cfos rabbit primary antibody (Calbiochem, Darmstadt, Germany), (1:20,000 dilution for $48 \mathrm{hr}$ at $\left.4^{\circ} \mathrm{C}\right)$. Following a series of $0.1 \mathrm{M}$ phosphate buffer washes, sections were stained using fresh blocking solution and Dylight 649 goat anti-rabbit secondary antibody (Jackson Immuno Research, West Grove, PA) (1:500 dilution for $24 \mathrm{hr}$ at $\left.4^{\circ} \mathrm{C}\right)$. Finally, sections were stained with DAPI (Invitrogen, Carlsbad, CA) (1:1,000 dilution for $5 \mathrm{~min}$ ) and mounted on slides. In some sections, excitatory neurons were identified using an anti-CaM Kinase II, $\alpha$ subunit, clone 6G9 mouse primary antibody (Millipore, Billerica, MA) (1:1,000 dilution for $48 \mathrm{hr}$ at $4^{\circ} \mathrm{C}$ ), followed by anti-Mouse IgG (Fab Specific)-Peroxidase secondary antibody made in goat (Sigma Aldrich, St. Louis, MO) (1:1,000 dilution for $24 \mathrm{hr}$ at $\left.4^{\circ} \mathrm{C}\right)$. In these sections staining was amplified using a TSA Cyanine 5 tyramide reagent amplification system (Perkin-Elmer, Waltham, MA) (1:50 dilution).

\section{Fluorescent Microscopy}

Sections from $-2.055 \mathrm{~mm}$ to $-2.25 \mathrm{~mm}$ posterior to bregma were used in all experiments. Within these coordinates we selected regions of interest in the dorsal hippocampus (DG, CA3, CA1), layer II/III of the lateral entorhinal cortex (ENTl), the basolateral nucleus of the amygdala (BLA), layer V/VI of the posterior parietal association area (PTLp), and 
layer II/III of the retrosplenial cortex (RSPv) based on previous studies (Figure 2A) (Kubik et al., 2012; Maviel, Durkin, Menzaghi, \& Bontempi, 2004; Reijmers et al., 2007; Tayler et al., 2011). Sections from $-0.88 \mathrm{~mm}$ to $-1.655 \mathrm{~mm}$ posterior to bregma were used in the analysis of a control brain region, the secondary motor cortex (MOs). Images were taken in the $\mathrm{z}$ plane at a frequency of $1-2 \mu \mathrm{m}$ and acquired using 40x (DG, CA3, CA1, ENTl) or 20x (BLA, PTLp, RSPv, MOs) objective lenses of a Nikon Eclipse 80i epiflourescence microscope and NIS Elements software (Nikon, Melville, NY). The Allen Brain Atlas (Allen Brain Atlas Resources, Seattle, WA) was used to identify each structure.

\section{Signal Quantification}

H2B-GFP and $\alpha$ CAMKII co-localization was determined by examining the overlap between pixels in the FITC (GFP) and CY5 ( $\alpha$ CAMKII staining) channels. Colocalization occurred when GFP staining in the nucleus was surrounded by a ring of cytosolic staining for $\alpha$ CAMKII. For all other quantification, the middle step of each image stack was used to determine the number of DAPI labeled nuclei expressing c-Fos and/or GFP. To aid in quantification, a Nikon Elements macro was written that highlighted potential signals in the c-Fos and GFP channels as regions of interest (ROI) based on signal intensity ( $\geq 2$ S.D. above mean intensity of the image channel), size ( $\leq$ $\mu \mathrm{m}^{2}$ area of a single DAPI labeled nuclei), and circularity. Signal quality and z stack position were examined by a blinded rater before cells were counted as positive. To determine colocalization, the c-Fos ROI-highlighted channel was merged with the GFP channel, allowing a rater to determine whether both signals were present in the same cell 
body. Cells were determined to be c-Fos positive, GFP positive, GFP $+c-F o s$ positive, or signal negative. The total number of DAPI cell bodies in each step was counted manually. Bilateral DG, CA1, CA3, ENT1, BLA, PTLp, RSPv, and MOs images were quantified from 5 sections per animal, giving a total of 10 images per region per animal. The percentage of DAPI-labeled cells containing c-Fos, GFP, or both was calculated for each image and then averaged to produce a single measurement in each region for each animal.

\section{Statistics}

Group differences were analyzed using one-way ANOVAs and planned comparisons (Fisher's PLSD). In some experiments, the percentage of double-labeled neurons $\left(\left(\frac{G F P+c-f o s}{D A P I}\right) \times 100\right)$ was compared to the percentage predicted by chance $\left(\left(\frac{G F P}{D A P I} \times \frac{c-f o s}{D A P I}\right) \times 100\right)$ using paired-samples t-tests. Statistical significance for all tests was set at $\mathrm{p}<0.05$. 


\section{RESULTS}

\section{Selective and persistent tagging of activated neurons in H2B-GFP TetTag mice}

To label cells during learning, we used transgenic mice that express a long lasting, activity-dependent form of GFP. In these mice, the tetracycline-transactivator (tTA) system for transgene regulation was combined with the c-Fos promoter to tag active neurons (Figure1A). In the absence of doxycycline (DOX), activation of the c-Fos promoter leads to expression of an H2B-GFP fusion protein, which is stable for several weeks after induction (Tayler et al., 2011; Tumbar et al., 2004). In the presence of DOX, H2B-GFP expression is prevented. H2B-GFP expression was widespread after context fear conditioning (Figure 1B, top) and was confined to excitatory neurons expressing aCamKII in the hippocampus (Figure 1B, bottom).

We first determined whether H2B-GFP TetTag mice could be used to selectively label neurons in the hippocampus that were active during context fear conditioning. Mice fear conditioned off DOX (OFF) showed greater H2B-GFP expression than homecage control animals $(\mathrm{HC})$ (main effect of group $\mathrm{F}(1,7)=25.28, \mathrm{p}<0.05$ ). Post-hoc tests (Fisher's PLSD) revealed that H2B-GFP expression was significantly elevated in CA1 and CA3 ( $p$ values $<0.05$ ) (Figure 1C). The percentage of labeled neurons was similar to levels observed in previous studies, indicating that H2B-GFP is a reliable indicator of cellular activity (Vazdarjanova et al., 2006; Zhang, Guzowski, \& Thomas, 2005). These results are consistent with a recent paper that showed activity-dependent labeling in fos-tTA transgenic mice recapitulates endogenous c-Fos expression (Liu et al., 2012). We also observed a $57 \%$ increase in the percentage of H2B-GFP positive neurons in the DG 
although this change was not statistically significant $(p=0.1)$. The lack of an effect was likely due to the small number of neurons that are typically activated in this region during learning (1-5\%) (Chawla et al., 2005; Leutgeb, Leutgeb, Moser, \& Moser, 2007;

Vazdarjanova et al., 2006). No H2B-GFP positive neurons were observed in DG, CA3 or CA1 in mice that were fear conditioned on DOX $(\mathrm{ON})$.

In the next experiment, we examined the stability of the H2B-GFP signal over time. TetTag mice were trained off DOX to label activated neurons with GFP. After training, the animals were put back on DOX for 2 or 14 days prior to brain extraction and quantification. We found equivalent H2B-GFP expression 2 and 14 days after training (no effect of day $F(1,7)<1$ ) (Figure 1D). These data indicate that the activity of tagged neurons can be followed for several weeks after context fear conditioning.

\section{Reactivation of neural networks during recent memory retrieval}

We next examined reactivation of H2B-GFP neurons during memory retrieval in several brain regions (Figure 2A) two days after training (Figure 2B). Regions were analyzed in coronal sections that ranged from $-2.05 \mathrm{~mm}$ to $-2.25 \mathrm{~mm}$ posterior to bregma. Neurons activated during context fear conditioning were labeled with H2B-GFP by removing DOX three days prior to training. The animals were put back on high concentration DOX immediately afterwards to prevent further H2B-GFP tagging. Two days later, the mice were tested in the same context and c-Fos expression was used to index cellular activity. H2B-GFP and c-Fos expression were quantified in DG, CA3, CA1 and the basolateral nucleus of the amygdala (BLA). The BLA receives direct projections from the 
hippocampus and is essential for context fear learning and expression (Cenquizca \& Swanson, 2007; Maren, 2003). Within the same AP coordinates, we also identified regions of interest in the lateral entorhinal cortex (ENTl), retrosplenial cortex (RSPv) and posterior parietal association area (PTLp). These regions were selected based on their contributions to spatial learning and their anatomical connections with the hippocampus (Andersen, Morris, Amaral, Bliss, \& O'Keefe, 2007; Burgess, 2008; Cenquizca \& Swanson, 2007; Cho \& Kesner, 1996; Kesner, 2009; Maviel et al., 2004). We also quantified reactivation in a control region (secondary motor cortex; MOs) that is not presumed to directly support context fear retrieval. Figures $2 \mathrm{C}$ and $2 \mathrm{D}$ illustrate the percentage of H2B-GFP and c-Fos expression that were observed across these brain regions two days after training.

Given that the hippocampus is required to retrieve recent context fear memories (Anagnostaras, Maren, \& Fanselow, 1999; Wiltgen et al., 2010), we expected to see reactivation of neurons (i.e. double labeling for H2B-GFP and c-Fos) in this region two days after training. Figure 3A shows the percentage of reactivated neurons in TetTag mice $\left(\left(\frac{G F P+c-f o s}{D A P I}\right) \times 100\right)$ relative to chance $\left(\left(\frac{G F P}{D A P I} \times \frac{c-f o s}{D A P I}\right) \times 100\right)$. Reactivation was significantly greater than chance in the DG (paired-samples t-test $\mathrm{t}(4)=3.54, \mathrm{p}<0.05$ ), CA1 $(\mathrm{t}(4)=3.86, \mathrm{p}<0.05)$, and BLA $(\mathrm{t}(4)=4.02, \mathrm{p}<0.05)$. Reactivation did not exceed chance in CA3 (paired-samples t-test, $p>0.05$ ). These results suggest that recent memory retrieval involves reactivation of neurons in the hippocampus and amygdala that were previously activated during learning. 
We next examined reactivation of cortical areas that are involved in spatial and contextual learning. Figure 3B shows the percentage of reactivated neurons in each region relative to chance $($ chance $=$ percent GFP $\mathrm{x}$ percent $\mathrm{c}-\mathrm{Fos})$. Reactivation was significantly greater than chance in the ENTl (paired-samples t-test $\mathrm{t}(4)=3.87, \mathrm{p}<0.05$ ), $\operatorname{RSPv}(\mathrm{t}(4)=7.53, \mathrm{p}<0.05)$, and PTLp $(\mathrm{t}(4)=4.20, \mathrm{p}<0.05)$. As expected, reactivation did not exceed chance in MOs (paired-samples t-test, $\mathrm{p}>0.05$ ). These results indicate that retrieval of a recently formed context fear memory involves widespread reactivation of cortical neurons that were engaged during learning.

\section{Memory retrieval is required for reactivation of H2B-GFP positive neurons}

The current data suggest that H2B-GFP positive neurons are reactivated during testing when animals retrieve a memory for the training context. However, it is possible that reactivation is driven by other stimuli that are present during training and testing (e.g. transport cues, experimenter, removal from the homecage). To examine this issue we performed two control experiments in which mice were trained but did not retrieve a memory. In the first experiment, mice were trained off DOX in context A (to induce GFP expression) but were not tested (i.e. sacrificed two days later from homecage, No Retrieval) (Figure 4, top). H2B-GFP expression did not differ between Retrieval and No Retrieval groups in CA1 or CA3, as both groups were trained identically in context A (p $>0.05$ ) (Figure 4A). We did observe a small increase in H2B-GFP tagging in the DG of No Retrieval mice (main effect of group F $(1,9)=10.93, \mathrm{p}<0.05$ ). Analysis of $\mathrm{c}-\mathrm{Fos}$ expression, however, revealed significant decreases in the DG (main effect of group F (1, $9)=9.01, p<0.05$ ), CA1 (main effect of group $F(1,9)=58.33, p<0.05)$ and CA3 of 
No Retrieval animals (main effect of group $F(1,9)=27.77, p<0.05$ ) (Figure 4B). This demonstrates that context testing induced the c-Fos expression observed in the AA Retrieval group.

Comparison of the percentage of reactivated cells in Retrieval and No Retrieval groups revealed a significant decrease in colocalization in CA1 (main effect of group F $(1,9)=$ $5.39, \mathrm{p}<0.05)($ Figure 5B) and a trend towards decrease in the DG $(F(1,9)=2.25, \mathrm{p}=$ 0.17) of No Retrieval animals. Comparison of reactivated neurons in CA3 did not reveal a difference between Retrieval and No Retrieval groups $(\mathrm{p}>0.05)$ (Figure 5B). To control for decreased c-Fos expression in No Retrieval mice, we also compared the percentage of reactivated neurons to that expected by chance. No Retrieval mice failed to show greater than chance reactivation in the DG or CA1 (paired-samples t-test, $\mathrm{p}$ values $>0.05$ ) (Figure 5A). Together, these results suggest that the reactivation observed in the DG and CA1 of Retrieval mice cannot be directly attributed to cues experienced during the off DOX period or training.

In the second control experiment, we quantified double labeling in mice that were trained in context A and tested in a different environment (context B) two days later (Figure 6, top). These animals were exposed to the same background cues (e.g. handling, transport, experimenter) during training and testing but should not retrieve a memory for context A. Consistent with this idea, freezing levels in context B ( $\bar{x}=5 \%$, SE 1.67) were significantly lower than those observed in our previous experiment when mice were trained and tested in context $\mathrm{A}(\bar{x}=30 \%$, SE 10.20) (main effect of group $\mathrm{F}(1,8)=5.85$, 
$\mathrm{p}<0.05$ ). Figures $6 \mathrm{~A}$ and $6 \mathrm{~B}$ show the percentage of neurons expressing H2B-GFP and c-Fos in the hippocampus, amygdala and cortex. Figures 6C and 6D illustrate the percentage of reactivated neurons relative to chance $($ chance $=$ percent GFP $\mathrm{x}$ percent $\mathrm{c}-$ Fos). Analysis of double labeling for H2B-GFP and c-Fos revealed that reactivation did not exceed chance in any of the brain regions examined (paired-samples t-test, all $\mathrm{p}$ values $>0.05$ ). These data suggest that reactivation of neural networks is only observed when memory is retrieved. However, there was a trend for reactivation in the BLA and CA1 suggesting that neurons in these regions may be sensitive to other stimuli that are present during training and testing. Nonetheless, together our experiments demonstrate that the main determinant of reactivation is re-exposure to the context in which footshock was previously administered. 


\section{RESULTS SUMMARY}

In this chapter, we observed that neurons active during training can be tagged with a stable form of GFP (H2B-GFP). Tagged neurons in the hippocampus, amygdala and cortex reactivated during memory retrieval two days after training. Reactivation was contingent on memory retrieval, as it did not exceed chance when mice were trained and tested in different environments. Reactivation was also impaired when mice were trained but not tested. Together, these results demonstrate for the first time that retrieval of a contextual fear memory induces the widespread reactivation of neural ensembles in the hippocampus, neocortex, and basolateral amygdala. 


\section{DISCUSSION}

The current data demonstrate that memory retrieval involves widespread reactivation of neural ensembles that were engaged during learning. Using H2B-GFP TetTag mice we found significant reactivation in several regions of the hippocampus, amygdala and cortex during the retrieval of a recently formed context fear memory. Reactivation was not observed when mice were trained and tested in different environments, and was also impaired when mice were trained but not tested. Similar results have been obtained in FISH studies where rats explored the same spatial environment twice within a 30-minute period (Guzowski et al., 1999; Kubik et al., 2012). Our experiments extend these findings to context fear conditioning using memory tests that were conducted days after training. Our results are also unique in that reactivation was observed even though behavioral responses were distinct during training and testing (i.e. exploration versus freezing). This implies that neural ensembles activated during exploration can be reactivated during subsequent memory retrieval when animals are immobile. To illustrate this point, Figure 7A shows the amount of activity observed during training and testing in context A (group AA Retrieval). Mice were significantly more active during the training session compared to the testing session (main effect of session $(1,4)=72.43$, $\mathrm{p}<0.05)$. The only reduction in activity that was observed during training occurred late in the session after footshocks were presented (bins 10-12). Figure 7B shows the amount of freezing observed during the same sessions. As expected, mice froze substantially more during the testing session compared to the training session (main effect of session $(1,4)=8.29, \mathrm{p}<0.05)$ 
It is possible that memory retrieval is not required for reactivation of $\mathrm{H} 2 \mathrm{~B}-\mathrm{GFP}$ positive neurons (e.g. exposure to the same sensory cues on two different occasions may simply activate a similar population of neurons). However, place cell work has found that repeated exploration of a spatial environment does not reactivate the same neurons unless plasticity mechanisms are engaged during learning. For example, if NMDARs are blocked during exploration of a novel context, place fields form but are not stable (Kentros et al., 1998). When the animal is subsequently returned to the same context, new place fields are observed as if the rat is in a different environment. Therefore, exploring the same physical environment is not sufficient to reactivate the same group of neurons. Instead, learning needs to take place during initial exploration so that the same spatial representation can be reactivated when the animals are returned to the environment. A recent paper found that blockade of $\mathrm{PKM} \zeta$, which impairs memory retrieval, also results in place cell remapping in a familiar environment (Barry et al., 2012). These results suggest that reactivation of neurons in the hippocampus is contingent on memory formation and retrieval.

Another piece of evidence comes from a recent study that found selective stimulation of neurons that were engaged during learning results in memory retrieval (Liu et al., 2012). In these experiments, a fos-tTA mouse was used to drive expression of channelrhodopsin (ChR2) in the DG during context fear conditioning. Subsequent stimulation of c-Fos positive neurons resulted in memory retrieval (i.e. freezing). This result is direct evidence that reactivated neurons play a role in memory retrieval. 


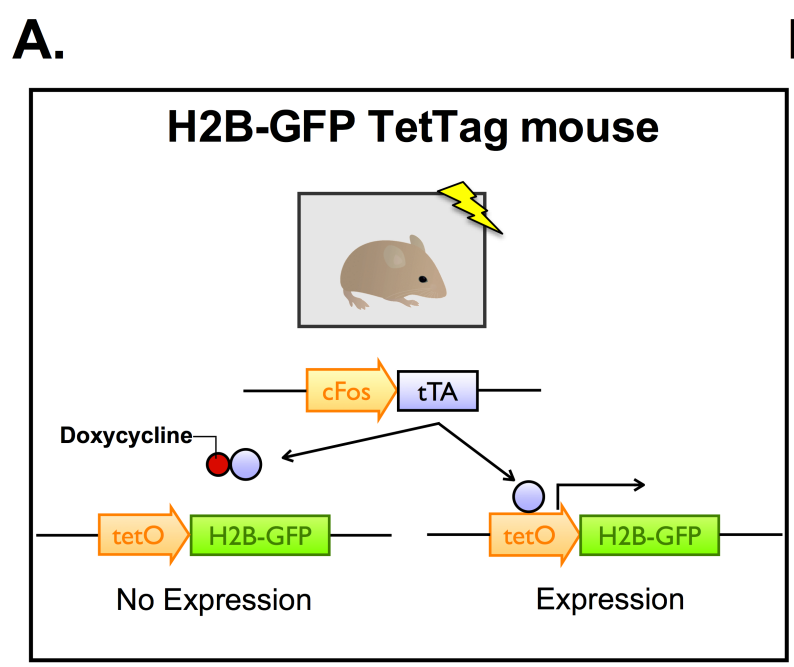

B.
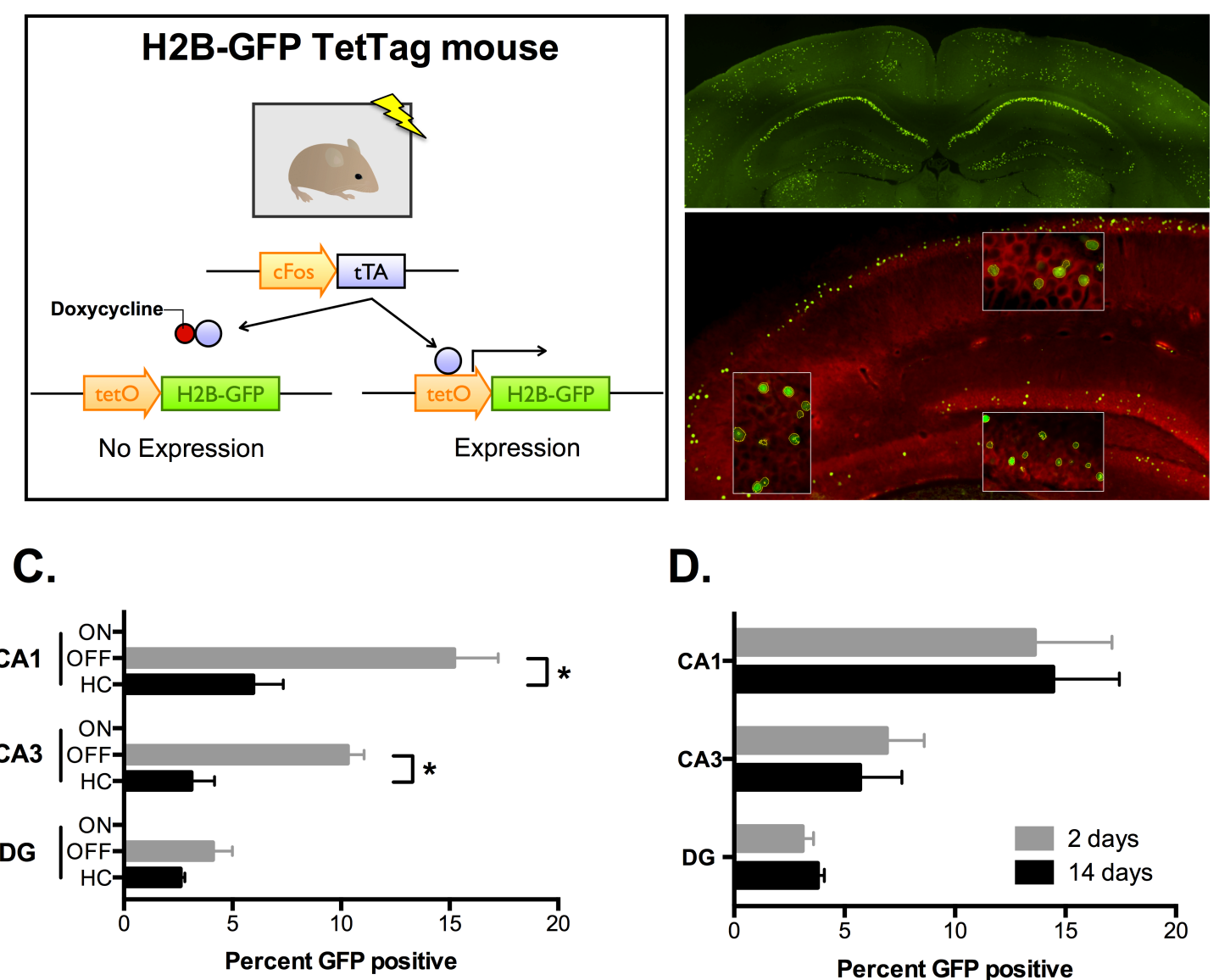

D.

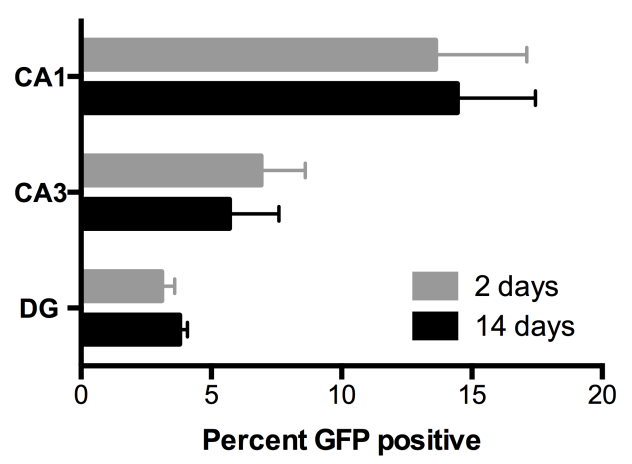

Figure 1. Selective and persistent tagging of active neurons in H2B-GFP TetTag mice. A) The tetracycline-transactivator (tTA) system for transgene regulation was combined with the c-Fos promoter to tag activated neurons. In the absence of doxycycline (DOX), activation of the c-Fos promoter leads to expression of an H2B-GFP fusion protein, which is stable for weeks after induction. In the presence of DOX, H2BGFP expression is prevented. B) Robust H2B-GFP expression was observed throughout the brain in mice that underwent context fear conditioning off DOX (top). Expression of H2B-GFP was limited to excitatory neurons labeled with $\alpha \mathrm{CAMKII}$ (red) in the hippocampus (bottom). The yellow outline indicates overlap between H2B-GFP positive nuclei and cytosolic $\alpha$ CAMKII staining. C) Mice fear conditioned off DOX (OFF) $(n=4)$ 
showed greater H2B-GFP expression than homecage control animals $(\mathrm{HC})(\mathrm{n}=5)$. H2BGFP expression was significantly elevated in CA1 and CA3. There was a numerical increase in the percentage of H2B-GFP positive neurons in the DG but this change did not reach statistical significance. No H2B-GFP expression was observed in mice fear conditioned on DOX $(\mathrm{ON})(\mathrm{n}=4)$. D) Two groups of mice were fear conditioned in the absence of DOX. Afterwards, the animals were put back on DOX for 2 days $(n=5)$ or 14 days $(n=4)$ before being sacrificed for immunohistochemistry. We observed robust expression of $\mathrm{H} 2 \mathrm{~B}-\mathrm{GFP}$ in DG, CA3 and CA1 that did not change over time. Error bars represent \pm SEM. $* \mathrm{p}<0.05$ 
A.

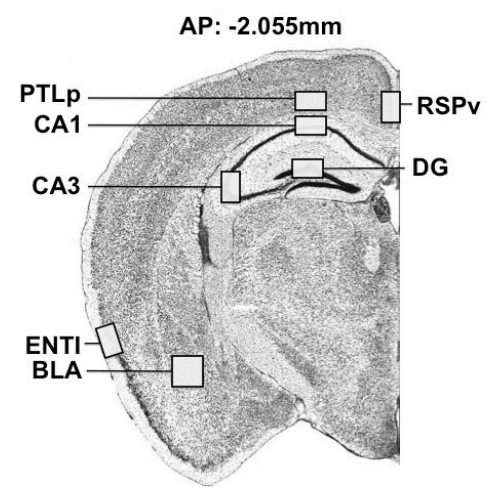

C.

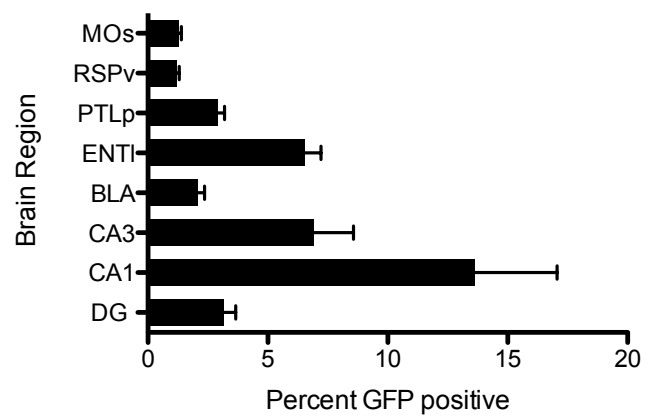

B.

\section{Behavioral Design}

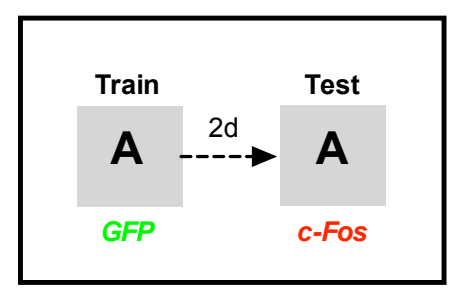

D.

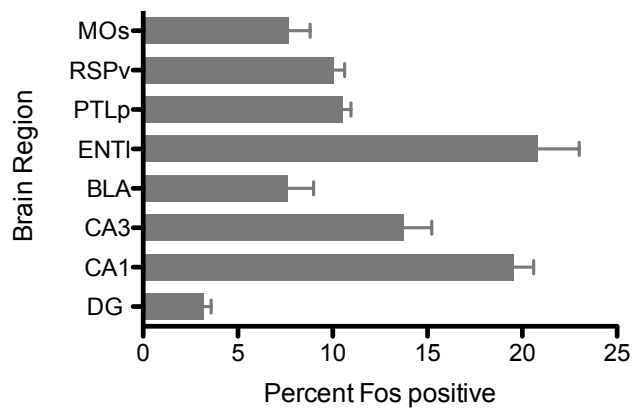

Figure 2. H2B-GFP and c-Fos expression after context fear conditioning and testing. A) A coronal section illustrating the brain regions analyzed for reactivation: dorsal hippocampus (DG, CA3, CA1), lateral entorhinal cortex (ENTl), basolateral amygdala (BLA), posterior parietal association area (PTLp) and retrosplenial cortex (RSPv). The secondary motor cortex (MOs) was also imaged $(-0.88 \mathrm{~mm}$ to $-1.655 \mathrm{~mm}$ to bregma). Image adapted from the Allen Reference Atlas. B) Behavioral procedure for the reactivation experiments. Mice underwent fear conditioning in context A off DOX to tag activated neurons with H2B-GFP. After training, animals were put back on DOX and tested 2 days later $(n=5)$. c-Fos expression during testing was used to identify activated neurons. Neurons double labeled with GFP and c-Fos were activated during training and testing. C) The percentage of neurons expressing H2B-GFP per brain region. D) The 
percentage of neurons expressing c-Fos following testing in context A. Error bars represent \pm SEM. ${ }^{*} \mathrm{p}<0.05$ 
A.

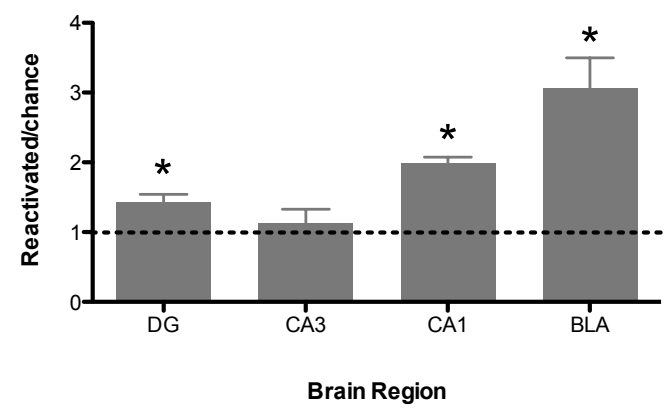

B.

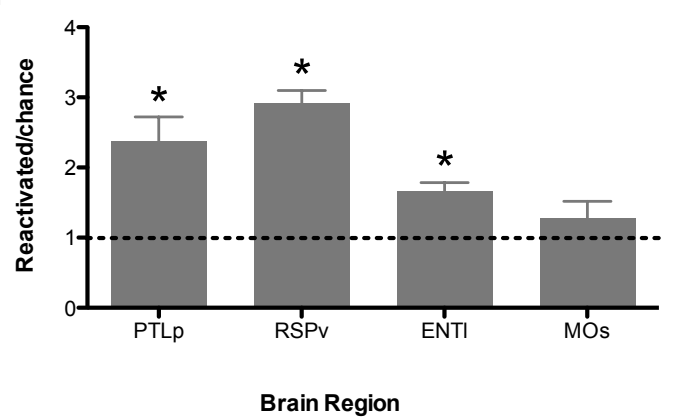

Figure 3. Reactivation of neurons during memory retrieval. A) TetTag mice were trained off DOX in context A and tested in the same environment 2 days later $(\mathrm{n}=5)$. The percentage of double-labeled neurons is shown relative to chance (percent H2B-GFP $\mathrm{x}$ percent $\mathrm{c}-\mathrm{Fos}$ ). Significant reactivation was observed in the DG, CA1 and BLA but not CA3. B) Significant cortical reactivation was observed in the PTLp, RSPv, and ENTl. Reactivation was not observed in a control region, the MOs. Error bars represent \pm SEM. $* \mathrm{p}<0.05$. 


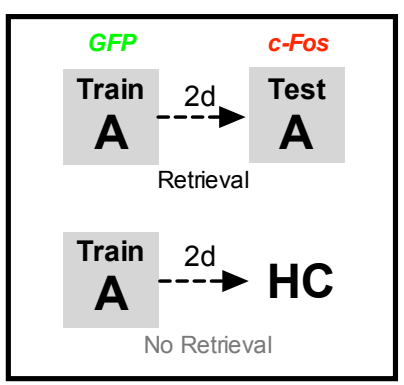

A.

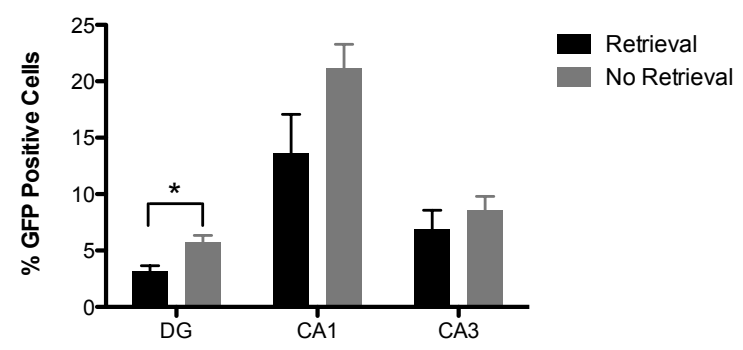

B.

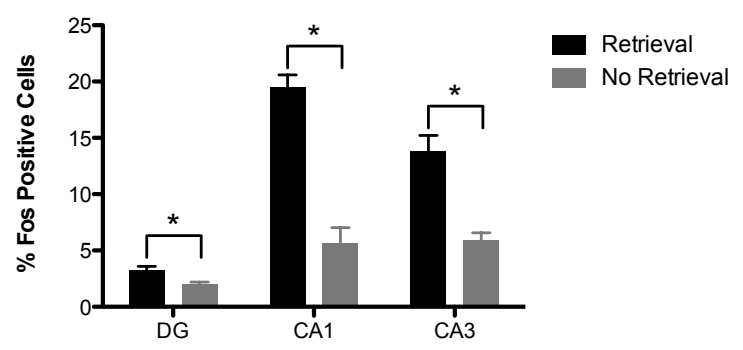

Figure 4. c-Fos expression is reduced in mice that are trained but not tested. TetTag mice were trained off DOX in context A and either tested (Retrieval, $\mathrm{n}=5$ ) or sacrificed directly from homecage (No Retrieval, $n=6$ ) 2 days later. A) The percentage of neurons expressing H2B-GFP across brain regions. B) The percentage of neurons expressing cFos across brain regions. c-Fos expression was significantly decreased in the DG, CA1, and $\mathrm{CA} 3$ of mice that were trained but not tested. Error bars represent \pm SEM. ${ }^{*} p<0.05$. 

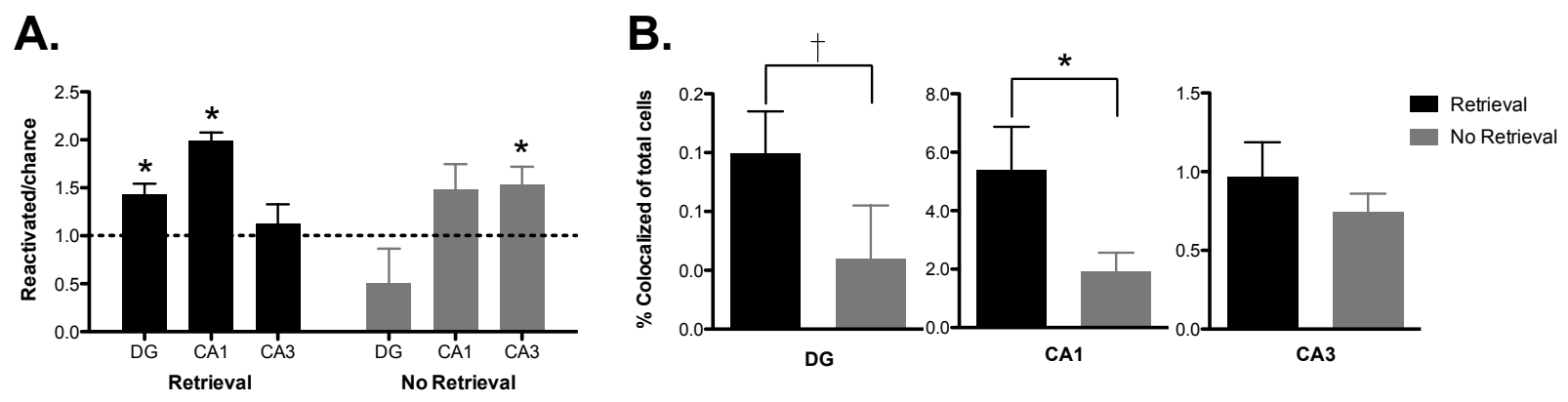

Figure 5. Reactivation is impaired in mice that are trained but not tested. A) The percentage of double labeled neurons $(\mathrm{H} 2 \mathrm{~B}-\mathrm{GFP}+\mathrm{c}-\mathrm{Fos})$ is shown relative to chance (percent H2B-GFP x percent c-Fos). No Retrieval mice $(n=6)$ failed to show greater than chance reactivation in the DG or CA1. B) The percentage of reactivated cells in CA1 was significantly decreased in No Retrieval animals. A numerical decrease in reactivation also occurred in the DG of mice trained but not tested. Error bars represent \pm SEM. ${ }^{*} p<$ 0.05 . 

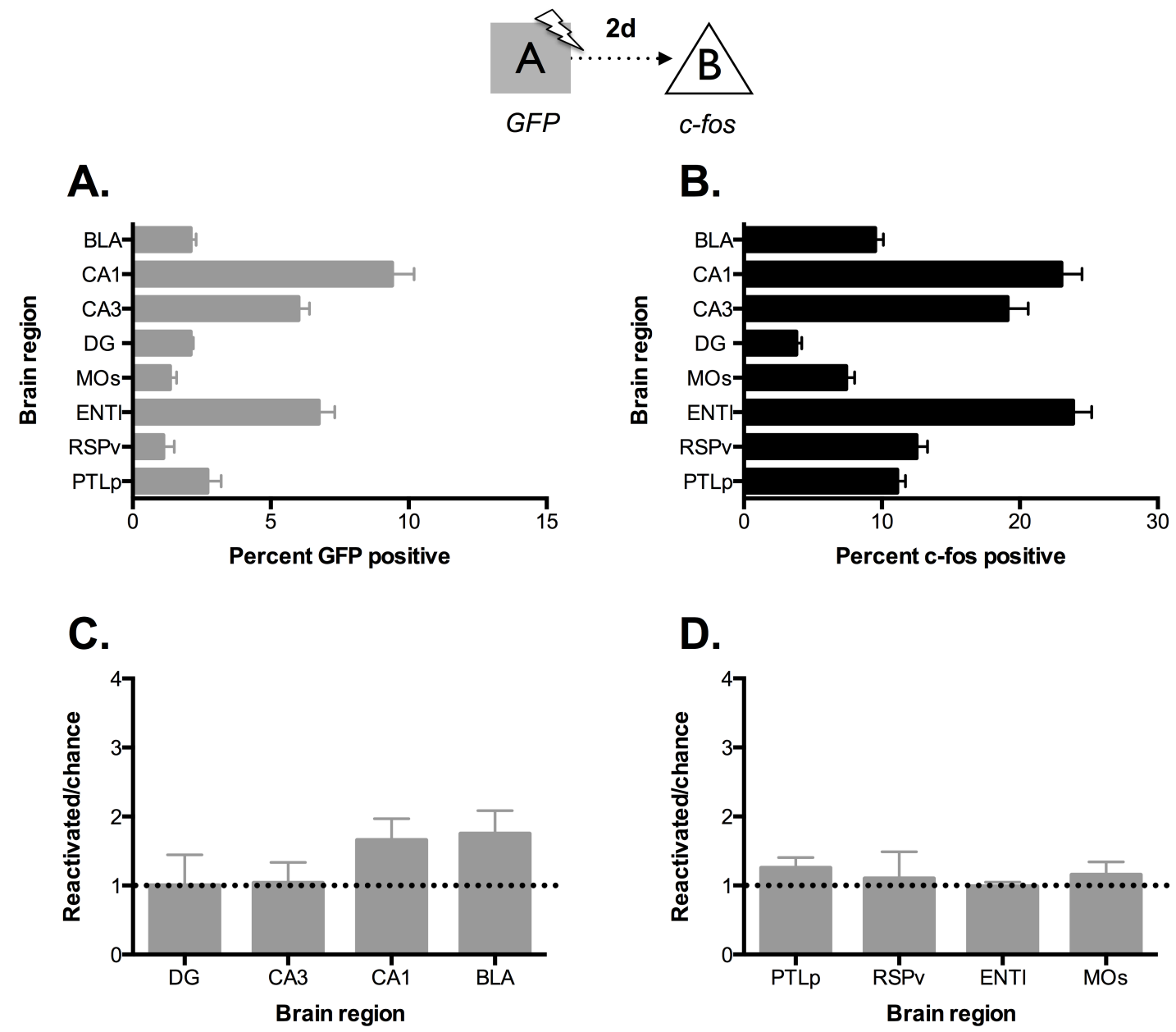

D.

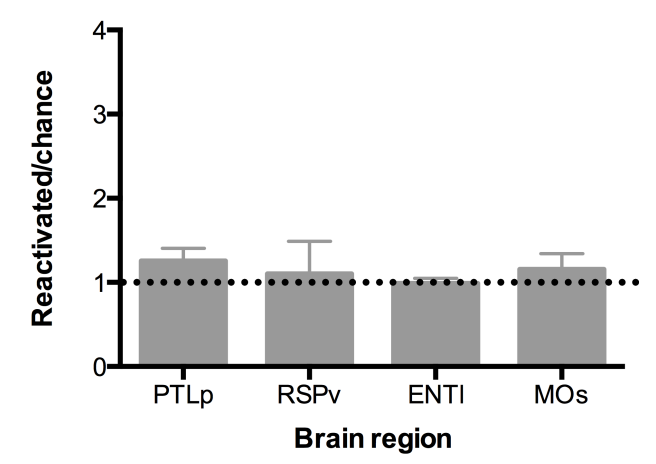

Figure 6: Memory retrieval is required for reactivation. Mice $(\mathrm{n}=5)$ were trained off DOX in context A and tested in context B two days later. A) The percentage of neurons expressing H2B-GFP across brain regions. B) The percentage of neurons expressing cFos across brain regions. C) The percentage of double labeled neurons (H2B-GFP + cFos) is shown relative to chance (percent H2B-GFP x percent c-Fos). Mice trained in context $\mathrm{A}$ and tested in context $\mathrm{B}$ did not show greater than chance reactivation in the hippocampus or BLA. D) Mice trained and tested in context B did not show greater than 
chance reactivation in any of the cortical regions examined. Error bars represent \pm SEM. $* \mathrm{p}<0.05$ 

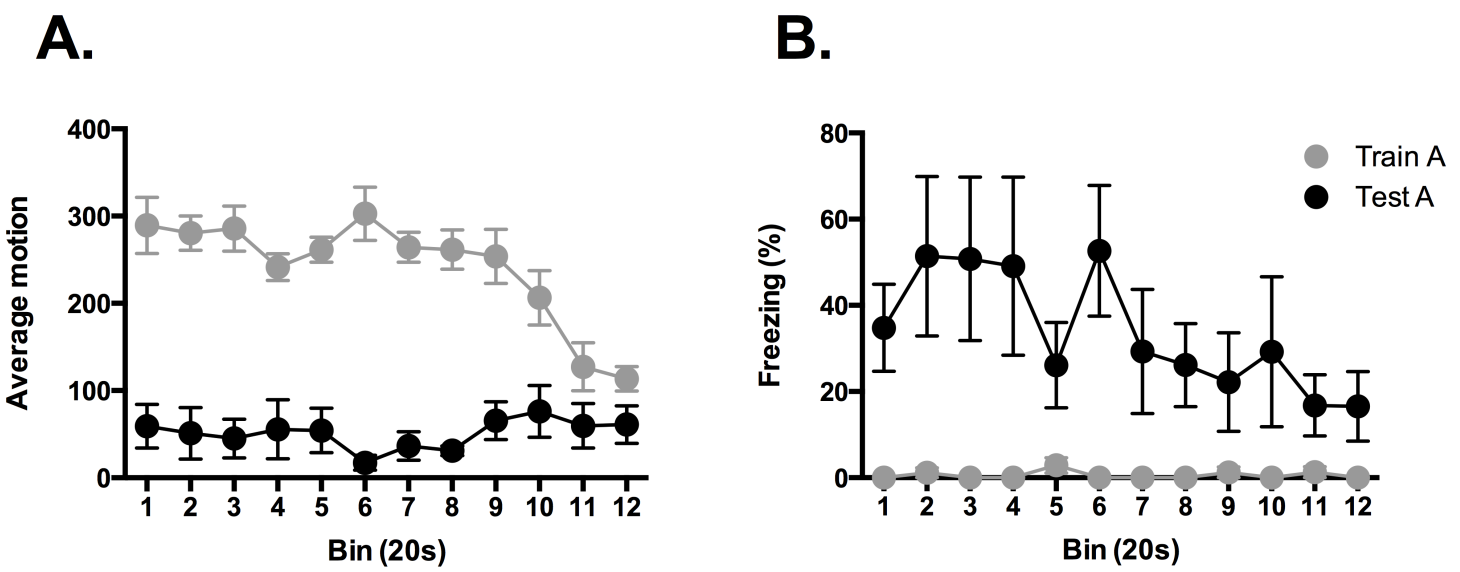

Figure 7: Exploration differences during training and testing. A) Average motion scores for mice trained and tested in context A. Mice were significantly more active during the training session compared to the testing session 2 days later. Activity only decreased during training after shock was presented (bins 10-12). B) Freezing data for the same training and testing sessions. There was significantly more freezing during the testing session compared to the training session. Error bars represent \pm SEM. 


\section{REFERENCES}

Anagnostaras, S. G., Maren, S., \& Fanselow, M. S. (1999). Temporally graded retrograde amnesia of contextual fear after hippocampal damage in rats: within-subjects examination. J Neurosci, 19(3), 1106-1114.

Anagnostaras, S. G., Wood, S. C., Shuman, T., Cai, D. J., Leduc, A. D., Zurn, K. R., . . . Herrera, G. M. (2010). Automated assessment of pavlovian conditioned freezing and shock reactivity in mice using the video freeze system. Front Behav Neurosci, 4.

Andersen, P., Morris, R. G., Amaral, D., Bliss, T., \& O'Keefe, J. (Eds.). (2007). The Hippocampus Book. Oxford: Oxford University Press.

Barry, J. M., Rivard, B., Fox, S. E., Fenton, A. A., Sacktor, T. C., \& Muller, R. U. (2012). Inhibition of protein kinase mzeta disrupts the stable spatial discharge of hippocampal place cells in a familiar environment. J Neurosci, 32(40), 1375313762.

Burgess, N. (2008). Spatial cognition and the brain. Annals of the New York Academy of Sciences, 1124, 77-97.

Cenquizca, L. A., \& Swanson, L. W. (2007). Spatial organization of direct hippocampal field CA1 axonal projections to the rest of the cerebral cortex. Brain research reviews, 56(1), 1-26.

Chawla, M. K., Guzowski, J. F., Ramirez-Amaya, V., Lipa, P., Hoffman, K. L., Marriott, L. K., . . Barnes, C. A. (2005). Sparse, environmentally selective expression of Arc RNA in the upper blade of the rodent fascia dentata by brief spatial experience. Hippocampus, 15(5), 579-586. 
Cho, Y. H., \& Kesner, R. P. (1996). Involvement of entorhinal cortex or parietal cortex in long-term spatial discrimination memory in rats: retrograde amnesia. Behavioral Neuroscience, $110(3), 436-442$.

Eichenbaum, H. (2004). Hippocampus: cognitive processes and neural representations that underlie declarative memory. Neuron, 44(1), 109-120.

Foudi, A., Hochedlinger, K., Van Buren, D., Schindler, J. W., Jaenisch, R., Carey, V., \& Hock, H. (2009). Analysis of histone 2B-GFP retention reveals slowly cycling hematopoietic stem cells. Nature biotechnology, 27(1), 84-90.

Frankland, P. W., \& Bontempi, B. (2005). The organization of recent and remote memories. Nat Rev Neurosci, 6(2), 119-130.

Frankland, P. W., Bontempi, B., Talton, L. E., Kaczmarek, L., \& Silva, A. J. (2004). The involvement of the anterior cingulate cortex in remote contextual fear memory. Science, 304(5672), 881-883.

Guzowski, J. F., McNaughton, B. L., Barnes, C. A., \& Worley, P. F. (1999). Environment-specific expression of the immediate-early gene Arc in hippocampal neuronal ensembles. Nat Neurosci, 2(12), 1120-1124.

Hall, J., Thomas, K. L., \& Everitt, B. J. (2001). Cellular imaging of zif268 expression in the hippocampus and amygdala during contextual and cued fear memory retrieval: selective activation of hippocampal CA1 neurons during the recall of contextual memories. The Journal of neuroscience, 21(6), 2186-2193.

Kanda, T., Sullivan, K. F., \& Wahl, G. M. (1998). Histone-GFP fusion protein enables sensitive analysis of chromosome dynamics in living mammalian cells. Current biology : 8(7), 377-385. 
Kentros, C., Hargreaves, E., Hawkins, R. D., Kandel, E. R., Shapiro, M., \& Muller, R. V. (1998). Abolition of long-term stability of new hippocampal place cell maps by NMDA receptor blockade. Science, 280(5372), 2121-2126.

Kesner, R. P. (2009). The posterior parietal cortex and long-term memory representation of spatial information. Neurobiology of learning and memory, 91(2), 197-206.

Kubik, S., Miyashita, T., Kubik-Zahorodna, A., \& Guzowski, J. F. (2012). Loss of activity-dependent Arc gene expression in the retrosplenial cortex after hippocampal inactivation: interaction in a higher-order memory circuit. Neurobiology of learning and memory, 97(1), 124-131.

Leutgeb, J. K., Leutgeb, S., Moser, M. B., \& Moser, E. I. (2007). Pattern separation in the dentate gyrus and CA3 of the hippocampus. Science, 315(5814), 961-966.

Liu, X., Ramirez, S., Pang, P. T., Puryear, C. B., Govindarajan, A., Deisseroth, K., \& Tonegawa, S. (2012). Optogenetic stimulation of a hippocampal engram activates fear memory recall. Nature. doi: 10.1038/nature11028

Lonergan, M. E., Gafford, G. M., Jarome, T. J., \& Helmstetter, F. J. (2010). Timedependent expression of Arc and zif268 after acquisition of fear conditioning. Neural plasticity, 2010, 139891. doi: 10.1155/2010/139891

Maren, S. (2001). Neurobiology of Pavlovian fear conditioning. Annual review of neuroscience, 24, 897-931.

Maren, S. (2003). The amygdala, synaptic plasticity, and fear memory. Annals of the New York Academy of Sciences, 985, 106-113.

Maviel, T., Durkin, T. P., Menzaghi, F., \& Bontempi, B. (2004). Sites of neocortical reorganization critical for remote spatial memory. Science, 305(5680), 96-99. 
Morris, R. G., Moser, E. I., Riedel, G., Martin, S. J., Sandin, J., Day, M., \& O'Carroll, C. (2003). Elements of a neurobiological theory of the hippocampus: the role of activity-dependent synaptic plasticity in memory. Philosophical transactions of the Royal Society of London. Series B, Biological sciences, 358(1432), 773-786.

Reijmers, L. G., Perkins, B. L., Matsuo, N., \& Mayford, M. (2007). Localization of a stable neural correlate of associative memory. Science, 317(5842), 1230-1233.

Schacter, D. L., Curran, T., Reiman, E. M., Chen, K., Bandy, D. J., \& Frost, J. T. (1999). Medial temporal lobe activation during episodic encoding and retrieval: a PET study. Hippocampus, 9(5), 575-581.

Tayler, K. K., Lowry, E., Tanaka, K., Levy, B., Reijmers, L., Mayford, M., \& Wiltgen, B. J. (2011). Characterization of NMDAR-Independent Learning in the Hippocampus. Front Behav Neurosci, 5, 28.

Tumbar, T., Guasch, G., Greco, V., Blanpain, C., Lowry, W. E., Rendl, M., \& Fuchs, E. (2004). Defining the epithelial stem cell niche in skin. Science, 303(5656), 359363.

Vazdarjanova, A., Ramirez-Amaya, V., Insel, N., Plummer, T. K., Rosi, S., Chowdhury, S., , . Barnes, C. A. (2006). Spatial exploration induces ARC, a plasticity-related immediate-early gene, only in calcium/calmodulin-dependent protein kinase IIpositive principal excitatory and inhibitory neurons of the rat forebrain. The Journal of comparative neurology, 498(3), 317-329.

Wiltgen, B. J., \& Fanselow, M. J. (2003). A model of hippocampal-cortical-amygdala interactions based on contextual fear conditioning. In K. Jeffery (Ed.), The neurobiology of spatial behaviour New York: Oxford University Press. 
Wiltgen, B. J., Zhou, M., Cai, Y., Balaji, J., Karlsson, M. G., Parivash, S. N., . . Silva, A. J. (2010). The hippocampus plays a selective role in the retrieval of detailed contextual memories. Curr Biol, 20(15), 1336-1344.

Zhang, W. P., Guzowski, J. F., \& Thomas, S. A. (2005). Mapping neuronal activation and the influence of adrenergic signaling during contextual memory retrieval.

Learning \& Memory, 12(3), 239-247. 
Chapter 3

Reactivation of Neurons During Remote Memory Retrieval 


\section{ABSTRACT}

In the previous chapter we demonstrated that recent memory retrieval is accompanied by widespread reactivation of neurons that were engaged during learning. Here we examined whether hippocampus, amygdala, and cortical reactivation continues to accompany memory retrieval when it occurs several weeks after training. We first observed that inactivation of the dorsal hippocampus prior to context testing impairs memory retrieval when performed two days, but not two weeks after training. This suggests that the systems underlying contextual memory retrieval reorganize within two weeks of learning. We next observed that neurons in the hippocampus (CA1 and CA3) and neocortex (ENT1 and PTLp) reactivate in TetTag mice trained and tested in the same environment across a 14-day interval. By comparing levels of reactivation at two days to fourteen days after training, we determined that patterns of reactivation shift in the hippocampus and amygdala but remain largely unchanged in the cortex. This finding suggests that hippocampal and amygdala circuits are modified after learning, while cortical networks remain stable over time. 


\section{INTRODUCTION}

Current models of memory predict that memory retrieval requires the reactivation of cortical ensembles that were engaged during learning. In Chapter 2, we demonstrated that retrieval of a recently acquired (2d) contextual fear memory results in the reactivation of H2B-GFP neurons tagged during training in the neocortex, hippocampus, and amygdala. Reactivation did not exceed chance in mice trained and tested in different environments and was reduced in mice that were trained but not tested. In this chapter, we examine whether reactivation accompanies retrieval of remote contextual fear memories.

The Standard Model of Systems Consolidation (SMC) states that the hippocampus binds and reactivates neocortical networks early after learning, but should become disengaged (i.e. not reactivated) over time as memory becomes stored in the cortex (Alvarez \& Squire, 1994). In contrast, Multiple Trace Theory (MTT) predicts that the hippocampus will always reactivate when episodic or contextual memories are retrieved, regardless of their age (Nadel \& Moscovitch, 1997). MTT states that repeated retrieval of an episodic memory creates new, redundant memory traces between the hippocampus and cortex. In this model, older memories may appear less susceptible to hippocampus damage than newer memories due to the presence of redundant hippocampus-dependent memory traces, not due to a disengagement of the hippocampus over time.

To test between these models, we examined reactivation in a group of H2B-GFP TetTag mice trained and tested in the same context over a longer time interval (14 days). Both models predict that neocortical neurons should reactivate during remote memory retrieval. However, the SMC predicts that hippocampal reactivation will decay over time, 
while MTT predicts that this structure will continue to reactivate even following a consolidation period. 


\section{METHODS}

\section{Subjects}

The TetTag mice used in these experiments are identical to those described in Chapter 2. Briefly, animals were generated by crossing transgenic mice that express a histone 2BGFP fusion protein controlled by the tetO promoter with mice that express tetracyclinetransactivator (tTA) protein under control of the c-Fos promoter (fos-tTA). B6/129 F1 hybrids were generated by breeding TetTag mice with 129S6 mice (Taconic, Hudson, NY). Mice were born and raised on DOX chow (40 mg/kg) to prevent H2B-GFP expression prior to experimental manipulations. All experiments were approved by the University of Virginia Animal Care and Use Committee.

\section{Apparatus}

The contextual fear conditioning equipment and chamber setup used in these experiments is also identical to that described in Chapter 2. Briefly, mice were trained in conditioning chambers (context A) that contained a stainless steel grid floor, overhead LED lighting, and a scanning CCD video camera. The chamber and drop pan were cleaned with $95 \%$ ethanol before each training session. Contextual fear memory was assessed by returning the mice to context $\mathrm{A}$ and measuring the freezing response.

\section{Behavioral Procedures}

TetTag mice on a B6/129 F1 hybrid background were taken off DOX for three days and trained with three unsignaled foot shocks $(0.5 \mathrm{~mA}, 2 \mathrm{~s})$. Immediately following training, all mice were given high concentration DOX chow $(1 \mathrm{~g} / \mathrm{kg})$ for 24 hours to rapidly 
suppress further GFP tagging and then remained on $40 \mathrm{mg} / \mathrm{kg}$ DOX chow for the duration of the study. Memory was assessed fourteen days later by returning the mice to the training context for five minutes and measuring the freezing response.

\section{Hippocampus Inactivation}

Hippocampus inactivation was performed in a separate group of B6/129 TetTag littermates lacking H2B-GFP. Mice were trained as described above in context A. Seven days prior to testing, mice underwent surgery to implant plastic guide cannulae (22 gauge; Plastics One, Roanoke, VA) bilaterally into the dorsal hippocampus. Mice were anesthetized with isoflurane and mounted in a stereotaxic apparatus (David Kopf Instruments, Tujunga, CA). Bregma and lambda were placed in the same horizontal plane. Cannulae were inserted through small burr holes and affixed with dental cement (Harry J. Bosworth Company, Skokie, IL) at the following positions relative to bregma (mm): AP: -2 , ML: \pm 1.5 , DV: -1 (from skull). Prior to testing in context A, mice were lightly anesthetized with isoflurane and injection cannulae (28 gauge) projecting $1 \mathrm{~mm}$ from the tip of the guide cannulae were used to infuse the AMPA/Kainate antagonist CNQX (Sigma Aldrich, St. Louis, MO) (3mM) or saline (0.9\%) into the dorsal hippocampus ( $0.5 \mu \mathrm{l} / \mathrm{side} ; 0.1 \mu \mathrm{l} /$ minute). Injectors remained in place for 2 minutes to allow for diffusion. Mice were tested in context A thirty minutes following the infusion. Cannulae placement was confirmed following testing using the Allen Reference Atlas (Dong, 2008).

\section{Immunohistochemistry}


Immunostaining procedures were identical to those described in Chapter 2. Briefly, H2BGFP TetTag mice were transcardially perfused ninety minutes after testing in context A with $4 \%$ PFA, followed by $24 \mathrm{~h}$ post-fixation. Free-floating $40 \mu \mathrm{m}$ coronal sections were prepared using a vibrotome, blocked, and stained using anti-cfos rabbit primary antibody (Calbiochem, Darmstadt, Germany), (1:20,000 dilution for $48 \mathrm{hr}$ at $\left.4^{\circ} \mathrm{C}\right)$ and Dylight 649 goat anti-rabbit secondary antibody (Jackson Immuno Research, West Grove, PA) (1:500 dilution for $24 \mathrm{hr}$ at $4^{\circ} \mathrm{C}$ ). Lastly, sections were stained with DAPI (Invitrogen, Carlsbad, CA) (1:1,000 dilution for $5 \mathrm{~min})$ and mounted on slides.

\section{Fluorescent Microscopy}

Microscopy techniques were identical to those described in Chapter 2. Sections from $2.055 \mathrm{~mm}$ to $-2.25 \mathrm{~mm}$ posterior to bregma were used to select regions of interest in the dorsal hippocampus (DG, CA3, CA1), layer II/III of the lateral entorhinal cortex (ENT1), the basolateral nucleus of the amygdala (BLA), layer V/VI of the posterior parietal association area (PTLp), and layer II/III of the retrosplenial cortex (RSPv) based on previous studies (Kubik, Miyashita, Kubik-Zahorodna, \& Guzowski, 2012; Maviel, Durkin, Menzaghi, \& Bontempi, 2004; Reijmers, Perkins, Matsuo, \& Mayford, 2007; Tayler et al., 2011). Sections from $-0.88 \mathrm{~mm}$ to $-1.655 \mathrm{~mm}$ posterior to bregma were used in the analysis of the secondary motor cortex (MOs). Images were taken in the z plane at

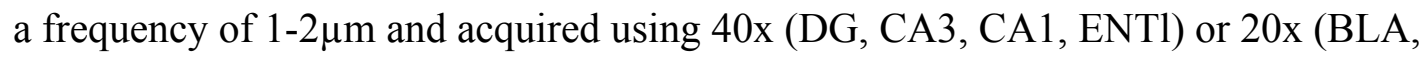
PTLp, RSPv, MOs) objective lenses of a Nikon Eclipse 80i epiflourescence microscope and NIS Elements software (Nikon, Melville, NY). 


\section{Signal Quantification}

Quantification procedures were identical to those described in Chapter 2. Briefly, a custom-written macro was used to identify c-Fos, GFP, or c-Fos + GFP positive cells in the middle step of each image stack. A blinded rater examined signal quality and z-stack position before cells were counted as positive. The total number of DAPI cell bodies in each step was counted manually. Once again, bilateral images for each region were quantified from 5 sections per animal, giving a total of 10 images per region per animal. The percentage of DAPI-labeled cells containing c-Fos, GFP, or both was calculated for each image and then averaged to produce a single measurement in each region for each animal.

\section{Statistics}

Group differences were analyzed using one-way ANOVAs and planned comparisons (Fisher's PLSD). In some experiments, the percentage of double-labeled neurons $\left(\left(\frac{G F P+c-f o s}{D A P I}\right) \times 100\right)$ was compared to chance $\left(\left(\frac{G F P}{D A P I} \times \frac{c-f o s}{D A P I}\right) \times 100\right)$ using pairedsamples t-tests. Statistical significance for all tests was set at $\mathrm{p}<0.05$. 


\section{RESULTS}

\section{H2B-GFP tagging persists two weeks after training}

Previously in Chapter 2, we demonstrated that H2B-GFP does not degrade within hippocampal subregions two weeks after training. To determine whether H2B-GFP signal is also stable in other analyzed brain regions (Figure 1A) we trained mice $(n=4)$ off DOX in context A. After training, animals were put back on high concentration $(1 \mathrm{~g} / \mathrm{kg})$ DOX for 24 hours, then regular DOX chow $(40 \mathrm{mg} / \mathrm{kg}$ ) for 13 days prior to testing and brain extraction (Figure 1B). We found equivalent H2B-GFP expression 2 and 14 days after training (no effect of day $\mathrm{F}(1,7)<1$ ) (Figure 1C). H2B-GFP expression was, however, reduced in the BLA, RSPv, and ACC in a pilot study of animals $(n=5)$ tested 28 days after training (data not shown). These data indicate that the activity of tagged neurons across the hippocampus, amygdala, and neocortex can be followed for up to two weeks after context fear conditioning. Examination of c-Fos expression induced by $14 \mathrm{~d}$ testing in context A revealed a difference between groups (main effect of group $\mathrm{F}(1,7)=$ 32.9, $\mathrm{p}<0.05)($ Figure 1D) that was limited to CA1 and RSPv (Fisher's PLSD $\mathrm{p}<0.05)$. In these regions, the number of c-Fos positive neurons increased 14 days after training. To control for these differences in expression, we compared the percentage of reactivated neurons $\left(\left(\frac{G F P+c-f o s}{D A P I}\right) \times 100\right)$ to that expected by chance $\left(\left(\frac{G F P}{D A P I} \times \frac{c-f o s}{D A P I}\right) \times 100\right)$.

\section{Reactivation of neural networks during remote memory retrieval}

Previous work indicates that context fear gradually becomes independent of the hippocampus after learning (Anagnostaras, Maren, \& Fanselow, 1999; Debiec, LeDoux, \& Nader, 2002; Kim \& Fanselow, 1992; Wiltgen et al., 2010). Therefore, we next 
determined whether the hippocampus is required for memory retrieval 2 and 14 days after training. To do this, we inactivated the dorsal hippocampus with the AMPAR antagonist CNQX prior to testing. Memory was assessed by measuring the freezing response, a species-specific defensive behavior observed in rodents (Anagnostaras et al., 2010). TetTag littermates lacking H2B-GFP were trained in context A and tested 2 or 14 days later in the same environment. Infusion of CNQX into the dorsal hippocampus 30 minutes prior to testing impaired memory retrieval at two days (main effect of group F (1, $24)=10.23, \mathrm{p}<0.05)$ but had no effect 14 days after training (no effect of group F (1, $22)<1)$ (Figure 2). These results suggest that the systems mediating context fear are reorganized within two weeks of training.

We next determined if hippocampal, amygdala, and cortical neurons reactivate when memory is retrieved 2 weeks after training. H2B-GFP positive animals $(n=4)$ were trained off DOX as described above and tested 14d later. Analysis of double labeling revealed that neurons in CA3 (paired-samples t-test $\mathrm{t}(3)=4.82, \mathrm{p}<0.05)$ and CA1 $(\mathrm{t}(3)=$ $5.69, \mathrm{p}<0.05$ ) were significantly reactivated during remote memory retrieval (Figure 3A). In contrast, reactivation in the BLA and DG did not exceed chance (paired-samples $t$-test, $p$ values $>0.05$ ). We next examined reactivation of cortical regions when memory was retrieved 2 weeks after training. Figure 3B shows the percentage of reactivated neurons in each region relative to chance. We found that reactivation was significantly greater than chance in the ENTl (paired-samples t-test $\mathrm{t}(3)=4.32, \mathrm{p}<0.05$ ) and PTLp $(\mathrm{t}(3)=8.59, \mathrm{p}<0.05)$ but not in the RSPv or MOs (paired-samples t-test, $\mathrm{p}$ values $>$ $0.05)$. 
These results deviated from our predictions about remote memory reactivation in two ways. First, we did not expect to find reactivation of hippocampal neurons two weeks after training given that this region was not required for memory retrieval at this time point. The fact that $\mathrm{CA} 3$ and $\mathrm{CA} 1$ were reactivated suggests that the hippocampus normally contributes to memory retrieval but that other structures can compensate when this region is inactivated. Recent studies are consistent with this idea (Goshen et al., 2011; Wiltgen et al., 2010; Winocur \& Moscovitch, 2011). Second, we did not observe reactivation of the BLA even though the amygdala is required for the retrieval of fear memories months and years after learning (Gale et al., 2004; Maren, Aharonov, \& Fanselow, 1996). Examination of our c-Fos expression data (Figure 1D) indicates that activity in the amygdala at 2 weeks $(9.9 \%)$ does not differ from that observed during memory retrieval at 2 days $(7.6 \%)$ (no effect of time $F(1,7)=1.31, \mathrm{p}>0.05)$. Therefore, it is possible that amygdala activity is required for remote memory retrieval even though reactivation of the same neurons that were engaged during learning is not. Alternatively, a smaller population of reactivated neurons (that did not exceed chance in the current experiment) may be sufficient to support memory retrieval at remote time points. To test the latter possibility we examined the relationship between BLA reactivation and freezing. There was a strong linear relationship between the percentage of reactivated neurons and the amount of freezing at 2 days $\left(\mathrm{r}^{2}=.81\right)$ and 2 weeks $\left(\mathrm{r}^{2}=\right.$ .62 ) that did not differ (no effect of time, $F(1,5)=3.14, p>0.05$ ). This suggests that reactivation of BLA neurons is related to the amount of freezing during recent and remote memory retrieval. This finding is consistent with a recently published paper that used a 
similar genetic system to examine reactivation of amygdala neurons following fear conditioning (Reijmers et al., 2007).

\section{Changes in regional reactivation over time}

To determine whether there were changes in the number of reactivated neurons over time we compared the percentage of H2B-GFP positive cells co-labeled with c-Fos

$\left(\frac{G F P+c-f o s}{\text { Total GFP }}\right)$ during the recent and remote memory tests (Figure 4A and 4B). Planned comparisons (Fisher's PLSD) found a significant reduction in the percentage of reactivated neurons in the BLA and DG during the remote memory test ( $p$ values $<0.05$ ), an increase in CA3 $(p<0.05)$ and no change in CA1 $(p>0.05)$.

In contrast, analysis revealed that the percentage of reactivated neurons in analyzed cortical regions did not change over time (all $\mathrm{p}$ values $>0.05$ ). Reactivation in RSPv did not exceed chance 14 days after training because of an increase in c-Fos expression (illustrated in Figure 1D), not because of a decline in the percentage of reactivated neurons. This result is consistent with a previous study that found increased expression of c-Fos in the retrosplenial cortex during the retrieval of remote spatial memories (Maviel et al., 2004). As shown in Figure 1D, we did not observe a change in c-Fos expression over time in any of the other cortical regions examined. Therefore, our data suggest that reactivation of cortical networks remains relatively stable after learning. 


\section{DISCUSSION}

In this chapter we observed that remote memory retrieval is accompanied by reactivation of the same neurons that were engaged during learning. However, patterns of regional reactivation were not identical between 2 and 14 days. Within the hippocampus, the percentage of reactivated neurons decreased in the DG, increased in CA3 and remained stable in CA1 over time. The continuous generation of new neurons in the DG may contribute to the loss of reactivation in this region. Recent data indicate that neurogenesis in the DG plays an essential role in the clearance of previously formed context fear memories (Feng et al., 2001; Kitamura et al., 2009). Based on these data, one would predict a gradual decline in the reactivation of DG neurons after learning. As the percentage of reactivated neurons decreased in the DG, we observed a corresponding increase in CA3. This finding may be related to the role that CA3 plays in pattern completion. Several studies have shown that context memories lose details and become less precise with the passage of time (Wiltgen \& Silva, 2007; Winocur, Moscovitch, \& Sekeres, 2007). This implies that remote memory retrieval requires reactivation of partially degraded information; a process that is known to depend on CA3 (Fellini, Florian, Courtey, \& Roullet, 2009; Nakazawa et al., 2002). Therefore, as reactivation of DG neurons decreases over time, memory retrieval may be supported by reactivation of CA3 and CA1 networks.

Neurons in the CA1 region of the hippocampus were reactivated during the retrieval of remote context fear memories. This result was unexpected given that the hippocampus was not required for memory retrieval 2 weeks after training (Figure 2). If memory can 
be retrieved without the hippocampus then why is this region still reactivated during retrieval? One possibility is that remote memory retrieval normally involves the hippocampus but can be mediated by other structures if this region is compromised. Two recent studies support this idea. The first showed that under some conditions, inactivation of the hippocampus does not impair context fear although it significantly alters the quality of memory that can be retrieved (Wiltgen et al., 2010). This result suggests that alternative brain regions can retrieve information that supports freezing if the hippocampus is compromised. The second study found that prolonged inhibition of the dorsal hippocampus produces compensatory changes in the anterior cingulate cortex (ACC) that are sufficient to support memory retrieval (Goshen et al., 2011). These data indicate that the hippocampus is: 1) required for recent memory retrieval and 2) contributes to, but is not essential, for remote memory retrieval.

We also observed robust reactivation of cortical regions involved in spatial and contextual learning (ENTl and PTLp) (Andersen, Morris, Amaral, Bliss, \& O'Keefe, 2007; Cho \& Kesner, 1996; Kesner, 2009; Maviel et al., 2004). Reactivation of H2BGFP positive neurons in these regions was similar during the retrieval of recent and remote context fear memories (Figure 4B). This result is consistent with both SMC and MTT models of memory that predict stable reactivation of cortical neurons over time. According to these models, consolidation involves a gradual strengthening of connections (either intra-cortical or cortico-hippocampal) between neurons that were active during learning (Alvarez \& Squire, 1994; Frankland \& Bontempi, 2005, Nadel \& Moscovitch, 
1997). Consequently, in both models cortical neurons should be reactivated during the retrieval of new and old memories as was observed in the current experiments.

In contrast to the cortex, there is disagreement about whether the hippocampus should be reactivated during the retrieval of remote memories. SMC predicts a gradual loss of hippocampal memory traces during consolidation while MTT argues that detailed contextual or spatial information is permanently stored in this structure (Frankland \& Bontempi, 2005; McClelland, McNaughton, \& O'Reilly, 1995; Moscovitch et al., 2005; Squire, 1992). Our data indicate that some regions of the hippocampus continue to be reactivated during retrieval even when cortical regions are capable of supporting memory. This result is consistent with the idea that hippocampal representations are maintained over time as complementary traces are established outside this structure (Moscovitch et al., 2005; Winocur \& Moscovitch, 2011). Our data also suggest that while cortical traces are being established, the hippocampal representation undergoes a qualitative change that results in decreased involvement of the DG and increased involvement of the CA3 region over time. 
A.
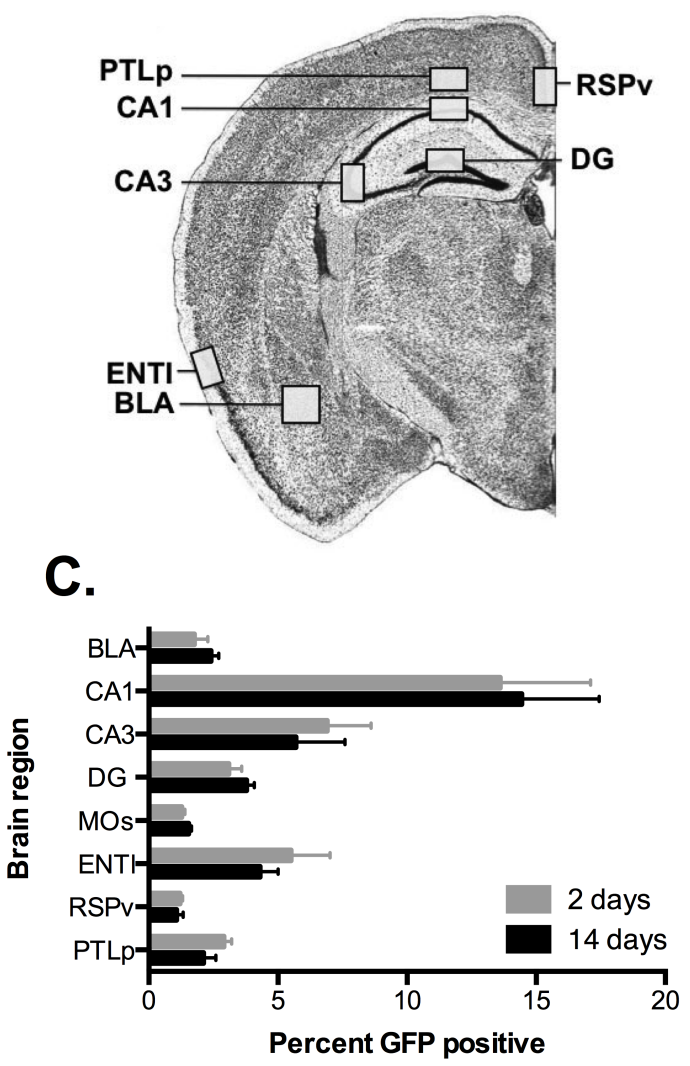

B.
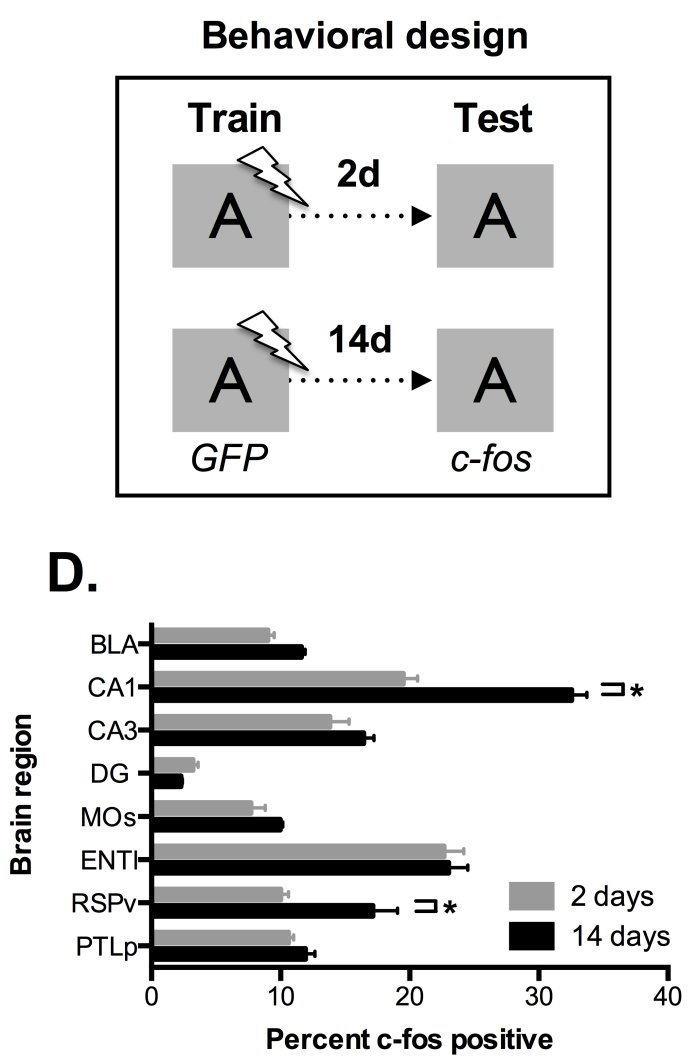

Figure 1. H2B-GFP and c-Fos expression are similar 2 and 14 days after training.

A) A coronal section (-2.05 $\mathrm{mm}$ posterior to bregma) illustrating the brain regions analyzed for reactivation: dorsal hippocampus (DG, CA3, CA1), lateral entorhinal cortex (ENTl), basolateral amygdala (BLA), posterior parietal association area (PTLp) and retrosplenial cortex (RSPv). Image adapted from the Allen Reference Atlas. B) Behavioral procedure for the reactivation experiment. Mice underwent fear conditioning in context A off DOX to tag activated neurons with H2B-GFP. After training, animals were put back on DOX and tested $2(n=5)$ or $14 d(n=4)$ later. c-Fos expression during testing was used to identify activated neurons. Neurons double labeled with GFP and cFos were activated during training and testing. C) The percentage of neurons expressing H2B-GFP was equivalent 2 and 14d after training. D) c-Fos expression was the same in 
most brain regions at 2 and 14d. It differed in CA1 and RSPv where expression was increased 14 days after training. Error bars represent \pm SEM. ${ }^{*} p<0.05$. 


\section{Behavioral Design}
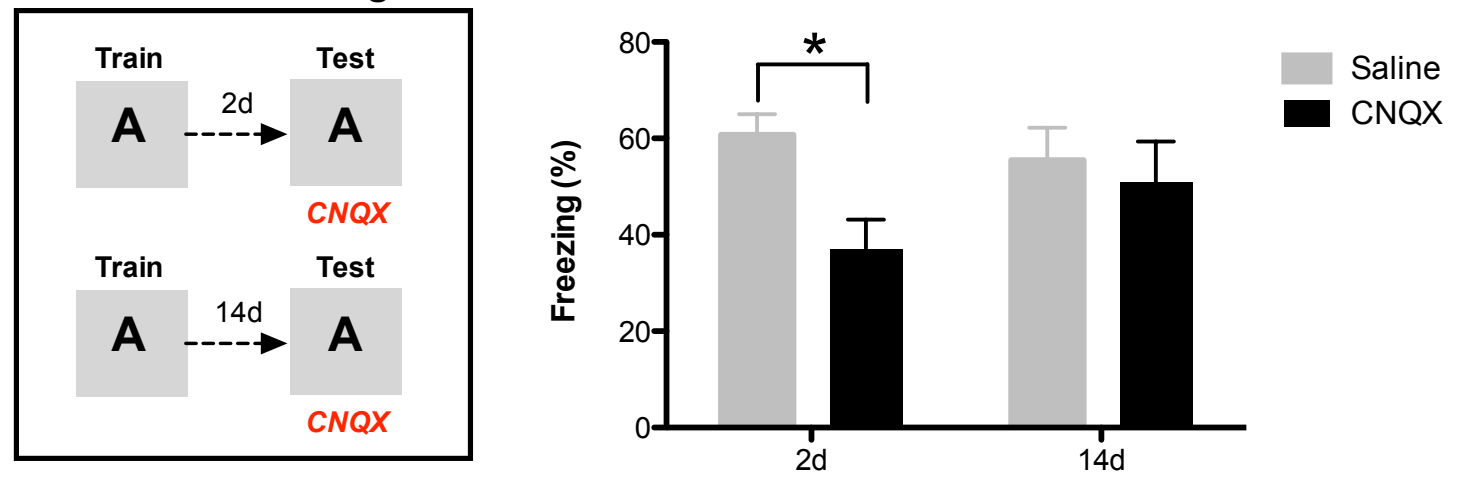

Figure 2. Contextual fear memory undergoes reorganization 14 days after training.

A) TetTag H2B-GFP-negative littermates were trained in context A and tested two days (Recent, saline $\mathrm{n}=13, \mathrm{CNQX} \mathrm{n}=13$ ) or 14 days later $($ Remote, saline $\mathrm{n}=12, \mathrm{CNQX} \mathrm{n}=$ 12) in the same environment. Infusion of CNQX into the dorsal hippocampus prior to testing impaired memory retrieval at 2 days but not 14 days after training. Error bars represent \pm SEM. $* p<0.05$ 
A.

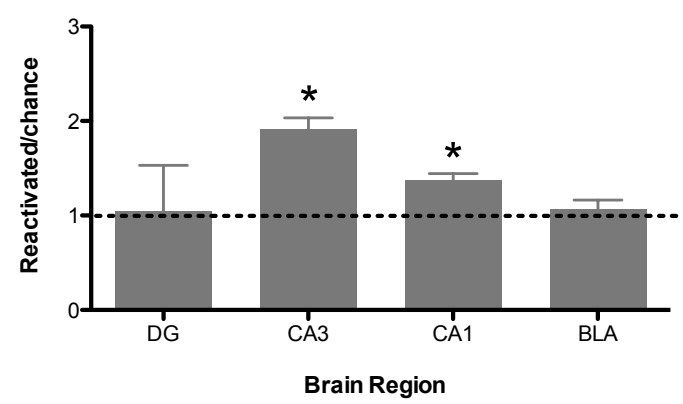

B.

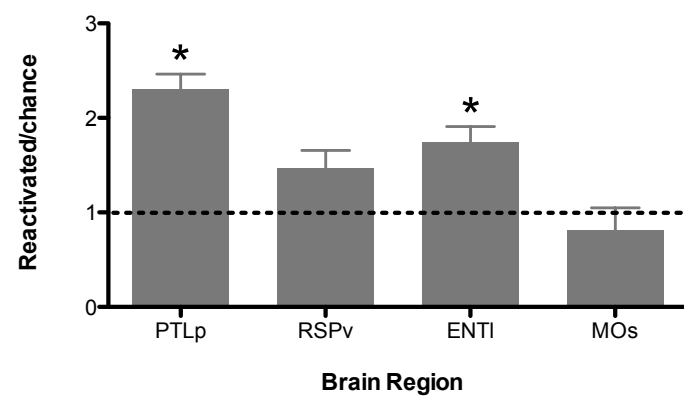

Figure 3. Reactivation of neurons during remote memory retrieval. A) TetTag mice were trained off DOX in context A and tested in the same environment 14 days later ( $\mathrm{n}=$ 4). The percentage of double-labeled neurons is shown relative to chance (percent H2BGFP $x$ percent c-Fos). Significant reactivation was observed in CA3 and CA1, but not in the DG or BLA. B) Two weeks after training reactivation exceeded chance in the ENT1 and PTLp but not RSPv or MOs. Error bars represent \pm SEM. *p $<0.05$. 
A.

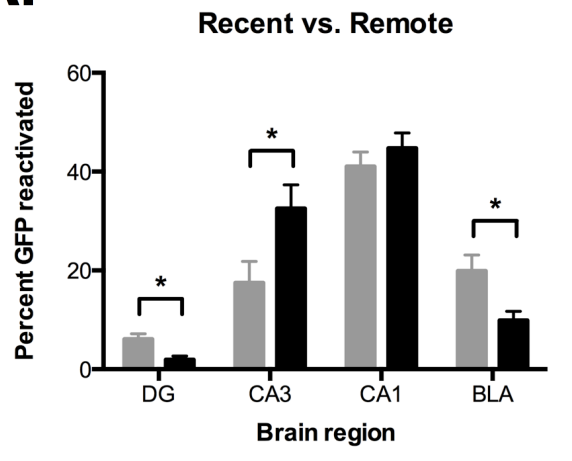

B.

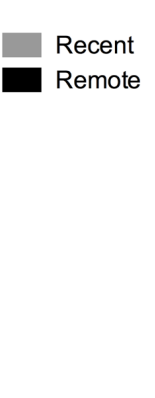

Recent vs. Remote

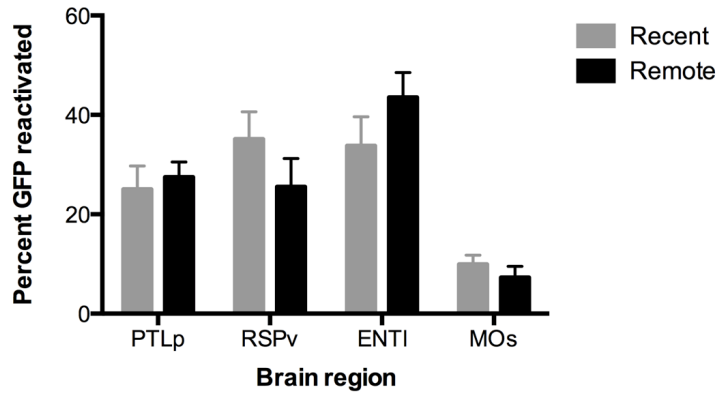

Figure 4. Changes in regional reactivation over time.

A) The percentage of $\mathrm{H} 2 \mathrm{~B}-\mathrm{GFP}$ positive neurons that were reactivated (i.e. co-labeled with c-Fos) is shown for mice tested 2 days (Recent, $n=5$ ) or 14 days (Remote, $n=4$ ) after training. The percentage of reactivated neurons decreased over time in the BLA and DG, increased in CA3 and remained unchanged in CA1. B) The percentage of H2B-GFP positive neurons that were reactivated did not change over time in any of the cortical regions examined. Error bars represent \pm SEM. ${ }^{*} \mathrm{p}<0.05$. 


\section{REFERENCES}

Alvarez, P., \& Squire, L. R. (1994). Memory consolidation and the medial temporal lobe: a simple network model. Proc Natl Acad Sci U S A, 91(15), 7041-7045.

Anagnostaras, S. G., Maren, S., \& Fanselow, M. S. (1999). Temporally graded retrograde amnesia of contextual fear after hippocampal damage in rats: within-subjects examination. J Neurosci, 19(3), 1106-1114.

Anagnostaras, S. G., Wood, S. C., Shuman, T., Cai, D. J., Leduc, A. D., Zurn, K. R., . . . Herrera, G. M. (2010). Automated assessment of pavlovian conditioned freezing and shock reactivity in mice using the video freeze system. Front Behav Neurosci, 4.

Andersen, P., Morris, R. G., Amaral, D., Bliss, T., \& O'Keefe, J. (Eds.). (2007). The Hippocampus Book. Oxford: Oxford University Press.

Cho, Y. H., \& Kesner, R. P. (1996). Involvement of entorhinal cortex or parietal cortex in long-term spatial discrimination memory in rats: retrograde amnesia. Behavioral Neuroscience, $110(3), 436-442$.

Debiec, J., LeDoux, J. E., \& Nader, K. (2002). Cellular and systems reconsolidation in the hippocampus. Neuron, 36(3), 527-538.

Dong, H. W. (2008). The Allen Reference Atlas. Hoboken, NJ: Wiley.

Fellini, L., Florian, C., Courtey, J., \& Roullet, P. (2009). Pharmacological intervention of hippocampal CA3 NMDA receptors impairs acquisition and long-term memory retrieval of spatial pattern completion task. Learning \& Memory, 16(6), 387-394.

Feng, R., Rampon, C., Tang, Y. P., Shrom, D., Jin, J., Kyin, M., . . Tsien, J. Z. (2001). Deficient neurogenesis in forebrain-specific presenilin-1 knockout mice is 
associated with reduced clearance of hippocampal memory traces. Neuron, 32(5), 911-926.

Frankland, P. W., \& Bontempi, B. (2005). The organization of recent and remote memories. Nat Rev Neurosci, 6(2), 119-130.

Gale, G. D., Anagnostaras, S. G., Godsil, B. P., Mitchell, S., Nozawa, T., Sage, J. R., .. . Fanselow, M. S. (2004). Role of the basolateral amygdala in the storage of fear memories across the adult lifetime of rats. $J$ Neurosci, 24(15), 3810-3815.

Goshen, I., Brodsky, M., Prakash, R., Wallace, J., Gradinaru, V., Ramakrishnan, C., \& Deisseroth, K. (2011). Dynamics of retrieval strategies for remote memories. Cell, 147(3), 678-689.

Kesner, R. P. (2009). The posterior parietal cortex and long-term memory representation of spatial information. [Review]. Neurobiology of learning and memory, 91(2), 197-206.

Kim, J. J., \& Fanselow, M. S. (1992). Modality-specific retrograde amnesia of fear. Science, 256(5057), 675-677.

Kitamura, T., Saitoh, Y., Takashima, N., Murayama, A., Niibori, Y., Ageta, H., .. . Inokuchi, K. (2009). Adult neurogenesis modulates the hippocampus-dependent period of associative fear memory. Cell, 139(4), 814-827.

Kubik, S., Miyashita, T., Kubik-Zahorodna, A., \& Guzowski, J. F. (2012). Loss of activity-dependent Arc gene expression in the retrosplenial cortex after hippocampal inactivation: interaction in a higher-order memory circuit. Neurobiology of learning and memory, 97(1), 124-131. 
Maren, S., Aharonov, G., \& Fanselow, M. S. (1996). Retrograde abolition of conditional fear after excitotoxic lesions in the basolateral amygdala of rats: absence of a temporal gradient. Behavioral Neuroscience, 110(4), 718-726.

Maviel, T., Durkin, T. P., Menzaghi, F., \& Bontempi, B. (2004). Sites of neocortical reorganization critical for remote spatial memory. Science, 305(5680), 96-99.

McClelland, J. L., McNaughton, B. L., \& O'Reilly, R. C. (1995). Why there are complementary learning systems in the hippocampus and neocortex: insights from the successes and failures of connectionist models of learning and memory. Psychol Rev, 102(3), 419-457.

Moscovitch, M., Rosenbaum, R. S., Gilboa, A., Addis, D. R., Westmacott, R., Grady, C., ... Nadel, L. (2005). Functional neuroanatomy of remote episodic, semantic and spatial memory: a unified account based on multiple trace theory. J Anat, 207(1), $35-66$.

Nadel, L., \& Moscovitch, M. (1997). Memory consolidation, retrograde amnesia and the hippocampal complex. Curr Opin Neurobiol, 7(2), 217-227.

Nakazawa, K., Quirk, M. C., Chitwood, R. A., Watanabe, M., Yeckel, M. F., Sun, L. D., . . . Tonegawa, S. (2002). Requirement for hippocampal CA3 NMDA receptors in associative memory recall. Science, 297(5579), 211-218.

Reijmers, L. G., Perkins, B. L., Matsuo, N., \& Mayford, M. (2007). Localization of a stable neural correlate of associative memory. Science, 317(5842), 1230-1233.

Squire, L. R. (1992). Memory and the hippocampus: a synthesis from findings with rats, monkeys, and humans. Psychol Rev, 99(2), 195-231. 
Tayler, K. K., Lowry, E., Tanaka, K., Levy, B., Reijmers, L., Mayford, M., \& Wiltgen, B. J. (2011). Characterization of NMDAR-independent learning in the hippocampus. Frontiers in Behavioral Neuroscience, 5(28).

Wiltgen, B. J., \& Silva, A. J. (2007). Memory for context becomes less specific with time. Learning \& memory, 14(4), 313-317.

Wiltgen, B. J., Zhou, M., Cai, Y., Balaji, J., Karlsson, M. G., Parivash, S. N., . . Silva, A. J. (2010). The hippocampus plays a selective role in the retrieval of detailed contextual memories. Curr Biol, 20(15), 1336-1344.

Winocur, G., \& Moscovitch, M. (2011). Memory transformation and systems consolidation. JINS, 17(5), 766-780.

Winocur, G., Moscovitch, M., \& Sekeres, M. (2007). Memory consolidation or transformation: context manipulation and hippocampal representations of memory. Nat Neurosci, 10(5), 555-557. 
Chapter 4

Involvement of the Hippocampus in Neocortical Reactivation 


\section{ABSTRACT}

The hippocampus is widely thought to drive the reactivation of neocortical circuits during the retrieval of episodic and contextual memories. However, there is little direct evidence to support this. In this chapter, we use transgenic mice to tag neocortical neurons during context fear conditioning and determine whether reactivation of these cells requires the hippocampus during memory retrieval. Hippocampus inactivation was performed either two or fourteen days after training. We observed that hippocampus inactivation two days after training impaired memory retrieval and reduced c-Fos expression and neural reactivation in CA1. Hippocampus inactivation at two days also reduced the degree of reactivation in the anterior cingulate and retrosplenial cortices and eliminated correlated cortical activity observed in control animals during retrieval. In contrast, inactivation at fourteen days did not result in impaired memory retrieval or significant decreases in cortical reactivation. Structural equation modeling revealed that inactivation at this later time point resulted unexpectedly in both an increase and decrease in coordinated cortical activity. This may reflect the emergence of cortex-based compensatory retrieval networks in the weeks following learning. 


\section{INTRODUCTION}

Most models of episodic and contextual memory assume that the hippocampus drives the reactivation of neocortical neurons during memory retrieval (Alvarez \& Squire, 1994; McClelland, McNaughton, \& O'Reilly, 1995; Moscovitch et al., 2005; O'Reilly \& Rudy, 2001; Squire, 1992; Teyler \& DiScenna, 1986; Teyler \& Rudy, 2007; Winocur \& Moscovitch, 2011). This idea was initially based on the rich anatomical connectivity between the hippocampus and the neocortex (Marr, 1971). The presence of direct projections from the hippocampus to the cortex is thought to allow the replay of hippocampal patterns observed during learning to stimulate the reactivation of cortical networks. Repeated reactivation of cortical neurons that were active during learning is thought to induce the formation of either new intra-cortical connections (Standard Model of Systems Consolidation, SMC) or redundant cortico-hippocampal traces (Multiple Trace Theory, MTT) that support long-term memory retrieval. Although electrophysiological studies demonstrate the replay of hippocampal and cortical firing patterns during periods of sleep and inactivity (Qin, McNaughton, Skaggs, \& Barnes, 1997; Siapas \& Wilson, 1998; Wilson \& McNaughton, 1994), a causal link between reactivation in the hippocampus and neocortex has not been demonstrated. As a result, it remains unknown whether the hippocampus is required for memory representations to reactivate within the cortex.

To address this issue, we used transgenic mice (H2B-GFP TetTag) to label neocortical neurons during learning. In these mice, context fear conditioning induces the expression of a long-lasting H2B-GFP protein (Tayler et al., 2011; Tayler, Tanaka, Reijmers, \& 
Wiltgen, 2013). In the previous chapters, we showed that H2B-GFP labeled neurons in the hippocampus, retrosplenial cortex and posterior parietal association area reactivate (i.e. co-label with c-Fos) when context fear memories are retrieved (Tayler et al., 2013). The purpose of the current study was to determine if reactivation of these neocortical regions requires the hippocampus.

The two major models of episodic and contextual memory (i.e. SMC and MTT) make different predictions about the importance of the hippocampus to cortical reactivation during remote memory retrieval. SMC proposes that over time newly formed intracortical connections allow the cortex to support memory retrieval without input from the hippocampus (Alvarez \& Squire, 1994). The SMC predicts, therefore, that inactivation of the hippocampus will affect the reactivation of cortical neurons and impair memory retrieval when it is performed early after learning, but not following a weeks-long consolidation period. This prediction is challenged, however, by Multiple Trace Theory's assertion that complete damage to the hippocampus impairs both recent and remote contextual memories (Nadel \& Moscovitch, 1997). According to MTT, the act of retrieving an episodic or contextual memory creates a new trace between the hippocampus and neocortex. Older memories, which have been retrieved more often than new memories, are more resilient to hippocampus damage because of the redundancy of their hippocampus-based connections, but cannot be retrieved if the hippocampus is completely compromised (Moscovitch et al., 2005). In contrast to SMC, MTT predicts that hippocampus inactivation will impair remote contextual memory retrieval and neocortical reactivation when performed following a consolidation period. 
Therefore, in this chapter we examine the impact of hippocampus inactivation on neocortical reactivation during retrieval at a recent (2d) and remote (14d) time point. Based on models of systems consolidation, we hypothesized that neocortical reactivation would rely on the hippocampus shortly after learning, but occur independently following a weeks-long consolidation period. We also anticipated that functional connectivity between cortical brain regions would be reduced when the hippocampus was inactivated two, but not fourteen days after training. Alternatively, if the MTT is more accurate, then we predicted that hippocampus inactivation at 14 days would impair neocortical reactivation and the ability of cortical regions to coordinate their activity during remote memory retrieval. 


\section{METHODS}

Mice

The animals used in these experiments are identical to the TetTag mice described in Chapters 2 and 3. TetTag mice on a B6/129 F1 hybrid background were born and raised on DOX chow (40 mg/kg) to prevent H2B-GFP expression prior to experiments. All procedures were performed on 10-12 week old male and female mice during the light phase of the 12-h light/dark cycle. Mice were single housed and given ad libitum access to food and water for the duration of the experiment. All experiments were approved by the University of Virginia Animal Care and Use Committee.

\section{Hippocampus Cannulation Procedure}

Ten days prior to training, all mice underwent surgery to implant plastic guide cannulae (22 gauge; Plastics One, Roanoke, VA) bilaterally into the dorsal hippocampus. Mice were anesthetized with isoflurane and mounted in a stereotaxic apparatus (David Kopf Instruments, Tujunga, CA). Bregma and lambda were placed in the same horizontal plane. To avoid damage to the posterior parietal association area (PTLp), cannulae were inserted at a $20^{\circ}$ angle through small burr holes and affixed with dental cement (Harry $\mathrm{J}$. Bosworth Company, Skokie, IL) at the following locations relative to bregma (mm): AP: $-2.25, \mathrm{ML}: \pm 3.0, \mathrm{DV}:-1$ (from skull). Animals were given seven days to recover prior to DOX chow removal.

\section{Contextual Fear Conditioning}


DOX was removed three days prior to training to label activated neurons with H2B-GFP. Mice were allowed to explore the context (context A) for three minutes prior to the onset of footshock ( 3 shocks, $0.5 \mathrm{~mA}, 2 \mathrm{~s}$ duration, $20 \mathrm{~s}$ ITI). Mice were returned to their homecages 30s after the last shock and immediately given high concentration DOX chow $(1 \mathrm{~g} / \mathrm{kg})$ to suppress further H2B-GFP expression. Memory was assessed two or fourteen days later by returning the mice to the training context for five minutes and measuring the freezing response. The contextual fear conditioning equipment used in these experiments is identical to that described in Chapters 2 and 3. Briefly, context A contained a singlegrid stainless steel floor, overhead LED lighting, and a scanning CCD video camera (Med Associates Inc., St. Albans, VT). The chamber and drop pan were cleaned with $95 \%$ ethanol prior to each session. Automated freezing measurements were obtained using the VideoFreeze system (Med Associates Inc., St. Albans, VT).

\section{Hippocampus Inactivation}

Prior to testing in context A, mice were briefly anesthetized with isoflurane and injection cannulae (28 gauge) projecting $1.25 \mathrm{~mm}$ from the tip of the guide cannulae were inserted to infuse the AMPA/Kainate antagonist CNQX (Sigma Aldrich, St. Louis, MO) (3mM) or saline $(0.9 \%)$ into the dorsal hippocampus $(0.75 \mu \mathrm{l} /$ side; $0.1 \mu \mathrm{l} /$ minute). Injectors remained in place for 2 minutes to allow for diffusion. Mice were tested sixty minutes following the infusion. Cannulae placement was confirmed using the Allen Reference Atlas (Dong, 2008).

\section{Immunohistochemistry and Quantification}


c-Fos Time Course:

We piloted the time course of c-Fos expression in CA1 to ensure that c-Fos positive cells were activated by the retrieval test and not by infusion handling. TetTag GFP negative littermates were removed from homecage, lightly anesthetized with isoflurane and placed briefly in a stereotaxic apparatus, transferred to a recovery box, and then remained in the infusion area for a total of ten minutes to simulate infusion handling. Mice were returned to homecage and transcardially perfused using 4\% PFA 90, 120, 150, or 180 minutes later ( $\mathrm{n}=4$ per time point). c-Fos immunostaining and macro-aided quantification of cFos positive cells proceeded as described below. Time point differences were analyzed using one-way ANOVAs and planned comparisons (Fisher's PLSD).

\section{Colocalization Staining Procedure:}

Mice were transcardially perfused using 4\% PFA ninety minutes after testing in context A. Following 24-h post-fixation, brains were sectioned, blocked, and stained for c-Fos (Calbiochem, Darmstadt, Germany, 1:20,000 dilution for $48 \mathrm{hr}$ at $4{ }^{\circ} \mathrm{C}$; Dylight 649 goat anti-rabbit secondary antibody, Jackson Immuno Research, West Grove, PA, 1:500 dilution for $24 \mathrm{hr}$ at $4^{\circ} \mathrm{C}$; DAPI, Invitrogen, Carlsbad, CA, 1:1,000 dilution for $5 \mathrm{~min}$ ). Complete staining procedures are identical to those described in Chapters 2 and 3. Imaging was conducted using the 20x objective of a Nikon $80 \mathrm{i}$ wide-field epiflourescence microscope and NIS Elements software (Nikon, Melville, NY). Bilateral CA1, PTLp, retrosplenial cortex (RSPv), anterior cingulate cortex (ACC), basolateral amygdala (BLA), central nucleus of the amygdala (CEA), and lateral entorhinal cortex (ENTl) images were quantified from 3 sections per animal, giving a total of 6 images per region 
per animal. The middle-most $2 \mu \mathrm{m}$ step of each image stack was used for quantification. A macro was used to identify c-Fos and GFP signals as regions of interest based on size, circularity, and intensity ( $\geq 2$ S.D. above mean intensity of the channel). Cells were determined to be c-Fos positive, GFP positive, GFP+c-Fos positive, or signal negative. The total number of DAPI-labeled cell bodies was counted manually. The percentage of cells containing c-Fos, GFP, or both was calculated for each image and then averaged to produce a single measurement in each region for each animal.

\section{Statistics}

Group differences were analyzed using one-way ANOVAs and planned comparisons (Fisher's PLSD). Reactivation (i.e. overlap) of neurons in each brain region was analyzed by first normalizing each mouse's percentage of double-labeled neurons to the average percentage of overlap in that brain region for all mice (i.e. overlap/mean). Normalized reactivation was then compared between groups using one-way ANOVAs. Simple linear regressions were used to examine the relationships between pairs of brain regions, and between GFP, c-Fos, or normalized colocalization and freezing. Statistical significance for all tests was set at $\mathrm{p}<0.05$.

\section{Structural Equation Modeling}

Structural equation modeling was performed using OpenMx and R software (Boker et al., 2012; Boker et al., 2011). We compared a null model, in which there was no anatomical structure imposed on the shared covariance (i.e. relatedness) of activity between cortical brain regions to an alternative model, in which anatomical connections were predicted to 
guide the strength and direction of covariance between regions. Our two models (null vs. alternative) were tested against the data to determine goodness of fit. Confirmatory structural equation models were generated using regional GFP, c-Fos, and normalized colocalization activity for each group. After determining whether Saline and CNQX group models differed at each time point ( $2 \mathrm{~d}$ and $14 \mathrm{~d})$, we tested the significance of individual anatomical connections in the models to identify the connection(s) responsible for the model difference. To do this, we compared nested models in which a) the anatomical path coefficients were allowed to be freely estimated between the Saline and CNQX groups (i.e. null model) and b) an alternative model, in which the anatomical path coefficients were fixed to be identical across groups (McIntosh \& Gonzalez-Lima, 1994). 


\section{RESULTS}

We began our experiments by performing a time course pilot for c-Fos expression to ensure that stimulation from the inactivation procedure (e.g. transport, handling, and cannula insertion) did not contaminate retrieval-driven c-Fos (Figure 1A). Expression in CA1 reached a maximum $\%$ of c-Fos positive cells ninety minutes following infusion handling and was no longer significantly different from homecage levels 150 minutes following handling $(\mathrm{p}>0.05)$. We therefore determined that mice tested 60 minutes after the infusion and perfused 90 minutes following testing would express retrieval-induced cFos (Figure 1B).

We next trained TetTag mice in a context fear chamber (context A) off DOX to label active neurons in the hippocampus and cortex with H2B-GFP. Two or fourteen days later, mice received an infusion of saline (2d: $\mathrm{n}=7,14 \mathrm{~d}: \mathrm{n}=6)$ or the AMPA/kainate glutamate receptor antagonist CNQX (2d: $\mathrm{n}=7,14 \mathrm{~d}: \mathrm{n}=7)$ into the dorsal hippocampus and were tested sixty minutes later (Figure 1C). CNQX was selected as the inactivation agent for its ability to inhibit hippocampus function without affecting fibers of passage (Winters \& Bussey, 2005).

\section{Inactivation two days after training}

We found that inactivating the dorsal hippocampus with CNQX two days after training impaired freezing during the context test (main effect of group $F(1,12)=16.12, p<$ 0.05) (Figure 2). Hippocampus inactivation also decreased c-Fos expression (main effect of group $\mathrm{F}(1,12)=13.71, \mathrm{p}<0.05)($ Figure $3 \mathrm{~A})$ and the percentage of reactivated cells 
in CA1 (main effect of group $F(1,12)=20.55, p<0.05$ ) (Figure 3B). CA1 H2B-GFP expression (which occurred during learning) was not affected $(\mathrm{p}>0.05)$.

We observed that CA1 activity during learning is an important predictor of subsequent memory retrieval. The percentage of H2B-GFP expressed in CA1 in saline animals correlated positively with the amount of freezing during testing two days later $(\mathrm{R}(5)=$ $\left.0.74, \mathrm{r}^{2}=0.55, \mathrm{p}=0.05\right)$. The percentage of reactivated neurons in CA1 in all mice also correlated positively with the amount of freezing during testing $\left(\mathrm{R}(12)=0.66, \mathrm{r}^{2}=0.44\right.$, $\mathrm{p}<0.05$ ) (Figure 4). These results suggest that reducing the reactivation of CA1 neurons via hippocampus inactivation impairs memory retrieval soon after learning has occurred.

We next examined the effects of hippocampus inactivation on a network of six extrahippocampal brain regions. As in Chapters 2 and 3, we examined the amygdala (basolateral amygdala (BLA) and added the central nucleus of the amygdala (CEA)), lateral entorhinal cortex (ENTl), retrosplenial cortex (RSPv) and posterior parietal association area (PTLp); regions important for spatial and fear learning (Kesner, 2009; Maviel, Durkin, Menzaghi, \& Bontempi, 2004). We also examined the anterior cingulate cortex (ACC), which plays an important role in the retrieval of context fear memories (Frankland, Bontempi, Talton, Kaczmarek, \& Silva, 2004; Goshen et al., 2011; Maviel et al., 2004; Vetere et al., 2011).

The percentage of H2B-GFP positive cells did not differ between saline and CNQX groups in any of the regions examined (all $\mathrm{p}$ values $>.05$ ). Surprisingly, despite a 
decrease in freezing behavior the percentage of BLA and CEA neurons positive for c-Fos did not decrease in CNQX animals as a result of hippocampus inactivation ( $\mathrm{p}$ values $>$ $.05)$. Hippocampus inactivation also did not affect c-Fos expression in the ACC ( $p>$ 0.05) (Figure 5A). However, we did observe a strong trend towards decreased c-Fos expression in the RSPv (main effect of group $\mathrm{F}(1,12)=4.071, \mathrm{p}=0.07$ ) (Figure 5A) and unexpected increases in PTLp and ENTl c-Fos expression following inactivation (PTLp: main effect of group $\mathrm{F}(1,12)=5.03, \mathrm{p}<0.05$; ENTl: main effect of group $\mathrm{F}(1,12)=$ 5.88, $\mathrm{p}<0.05$ ) (Figure 5A). Decreased RSPv c-Fos expression was not unexpected, given that recent work by Guzowski and colleagues demonstrates decreased Arc immediate early gene expression in the RSPv following inactivation of the dorsal hippocampus using tetrodotoxin (Kubik, Miyashita, Kubik-Zahorodna, \& Guzowski, 2012). In both the current experiment and Guzowski study it may be that loss of direct output from dorsal CA1 projections to layer $2 / 3$ of the RSPv results in decreased cortical activity (Cenquizca \& Swanson, 2007).

We next examined changes in colocalization. We observed no group differences in normalized colocalization (i.e. percentage of double-labeled neurons normalized to the average percentage of overlap in that brain region for all mice) in the BLA, ENT1, or PTLp (all p values $>0.05$ ). We did, however, observe an unexpected increase in reactivation in the CEA of CNQX mice (main effect of group $F(1,12)=9.45, p<0.05$ ). Analysis of cortical reactivation revealed a significant reduction in normalized colocalization in the ACC (main effect of group $\mathrm{F}(1,12)=4.63, \mathrm{p}<0.05$ ) and a strong trend towards reduction in the RSPv (main effect of group $F(1,12)=3.19, p=.10$ ) 
(Figures 5B \& 6). These data indicate that reactivation of cells in the ACC and RSPV during memory retrieval relies on the hippocampus two days after training.

Finally, we examined correlated activity between individual brain regions during memory retrieval. As expected, regional correlations for the percentage of H2B-GFP positive cells were similar in CNQX and saline animals, as the hippocampus was active during training in both groups (Figure 7). We observed positive relationships between GFP expression in the PTLp and RSPv (Saline: $\mathrm{R}(4)=0.93, \mathrm{r}^{2}=0.87, \mathrm{p}<0.05, \mathrm{CNQX}$ : $\mathrm{R}(5)=0.64, \mathrm{r}^{2}=$ $0.41, p=0.1$ ) and between the RSPv and ACC (Saline: $\mathrm{R}(4)=0.61, \mathrm{r}^{2}=0.37, \mathrm{p}=0.1$, CNQX: $\left.\mathrm{R}(5)=0.68, \mathrm{r}^{2}=0.46, \mathrm{p}=0.1\right)$. Both groups also exhibited strong, positive correlations for the percentage of c-Fos positive cells between the BLA and CEA (Saline: $\mathrm{R}(4)=0.90, \mathrm{r}^{2}=0.81, \mathrm{p}<0.05$, CNQX: $\left.\mathrm{R}(5)=0.76, \mathrm{r}^{2}=0.58, \mathrm{p}<0.05\right)$ (Figure 7). This regional correlation was not observed in the GFP data, demonstrating that it emerged after training and therefore may be specific to memory retrieval. The presence of this relationship in CNQX mice suggests that coordination between the BLA and CEA during retrieval is not dependent on the hippocampus.

Group differences emerged, however, when we analyzed the degree of reactivation (i.e. normalized colocalization). Normalized colocalization was positively correlated between the PTLp and RSPv during memory retrieval in saline animals $\left(\mathrm{R}(4)=0.81, \mathrm{r}^{2}=0.66, \mathrm{p}<\right.$ 0.05) (Figure 8). This supports the idea that coordinated cortical activity accompanies memory retrieval (Hoffman \& McNaughton, 2002; Squire, 1992). Interestingly, this cortical correlation was eliminated by hippocampus inactivation $\left(R(5)=0.35, r^{2}=0.12\right.$, $p$ 
$>0.05$ ) (Figure 8). This indicates that the hippocampus plays an important role in coordinating cortical activity during memory retrieval.

To examine correlated activity across multiple brain regions, we next performed structural equation modeling (SEM). Unlike linear regression analysis, which limits one to examining relationships between individual brain regions, SEM allows us to follow changes in coordinated cortical activity across multiple brain regions. We estimated changes in functional connectivity between hippocampal, cortical, and amygdala regions linked by known anatomical pathways (Andersen, 2007; Cenquizca \& Swanson, 2007) (Figure 10). By structuring the model using known neuroanatomical connections, we could conclude that any significant correlations found between our brain regions were in fact anatomically viable (Addis \& McAndrews, 2006). We observed that Saline and CNQX models generated from GFP and c-Fos data did not differ based on factor loadings (i.e. path coefficients, $p$ values $>0.05$ ). However, models generated using normalized colocalization data did differ based on path coefficients $(\mathrm{p}<0.05)$. Change in the path coefficient from PTLP $\rightarrow$ RSPV following inactivation was found to drive the difference in the Saline and CNQX models $(\mathrm{p}<0.05)$ (Figure 10). Together, our correlation and modeling data suggest that inactivating the hippocampus two days after training impairs both coordinated cortical activity and functional connectivity during memory retrieval.

\section{Inactivation fourteen days after training}

Inactivating the dorsal hippocampus fourteen days after training did not significantly impair freezing during the context test $(\mathrm{p}>0.05)$ (Figure 11). This suggests that the systems supporting contextual memory retrieval are reorganized two weeks after training. 
Hippocampus inactivation did cause a numeric (21.65\%) decrease in CA1 c-Fos expression, however the decrease was not significant at this time point $(\mathrm{p}>0.05)$ (Figure 12A). This appears to be the result of a sharp decrease in saline CA1 c-Fos expression at 14 days (main effect of day $\mathrm{F}(1,11)=7.59, \mathrm{p}<0.05$ ) rather than an infusion failure, as CNQX c-Fos expression did not differ between 2 and 14 days ( $p>0.05$ ). Decreased 14 day c-Fos expression was not a general phenomenon: no other brain region sampled exhibited decreased c-Fos in saline controls. Inactivating the dorsal hippocampus also reduced the percentage of reactivated cells in CA1 by $31.75 \%$ (Figure 12B), however this decrease was not significant. This may be due once again to a decrease in the percentage of reactivated CA1 cells in the saline group at this time point (main effect of day F $(1,11)$ $=20.14, \mathrm{p}<0.05)$, as CNQX groups did not differ between 2 and 14 days $(\mathrm{p}>0.05)$. Although CA1 GFP expression did not differ between saline $(n=6)$ and CNQX mice ( $n$ $=7)(p>0.05)($ Figure 12A), correlation analysis failed to produce significant relationships between 14d CA1 GFP or normalized colocalization and freezing. Together, these results could serve as evidence of CA1 disengagement in the retrieval process following consolidation (Frankland \& Bontempi, 2005; Frankland et al., 2004).

We next examined the effects of hippocampus inactivation at 14 days on the BLA, CEA, ENTl, ACC, RSPv, and PLTp. Once again, GFP expression did not differ between saline and CNQX groups in these regions (all $p$ values $>0.05$ ). However, at 14 days we did observe extreme H2B-GFP signal degradation in the BLA. As a result, it is difficult to interpret BLA data for this time point and we have excluded BLA pathways from the 14day SEM. 
Hippocampus inactivation did not reduce c-Fos expression in the ACC, RSPv, or PTLp (all $p$ values $>0.05)$ (Figure 13A). Inactivation also did not reduce reactivation of cells in the ACC, RSPv, or PTLp (all p values > .05) (Figure 13B). Together, these data could support the SMC: indicating that retrieval-driven cortical reactivation is impaired by hippocampus inactivation when it is performed two, but not fourteen days after training. However, this interpretation of the data is undermined by the fact that CNQX groups did not experience a significant increase in the percentage of colocalized cells in any cortical region between 2 and 14 days (all $p$ values $>0.05$ ). Instead, we observed a numeric (though insignificant) reduction from 2 to 14 days in ACC and RSPv colocalization in the saline group (all p values $>0.05$ ). Therefore, we cannot rule out that the lack of impaired cortical reactivation at $14 \mathrm{~d}$ could have been driven by reduced reactivation in the saline group and not by the emergence of hippocampus-independent cortical reactivation in CNQX mice.

We next examined correlated activity between individual brain regions during memory retrieval (Figures 14-16). GFP correlations were similar across saline and CNQX groups, with the exception of a correlation between PTLp and RSPv in CNQX animals $(\mathrm{R}(5)=$ $\left.0.88, \mathrm{r}^{2}=0.79, \mathrm{p}<0.05\right)$ (Figure 14). When we analyzed correlations in 14-day c-Fos expression, we observed the surprising presence of correlated activity between PTLp and $\operatorname{RSPv}\left(R(5)=0.82, r^{2}=0.67, p<0.05\right)$, and between CA1 and $\operatorname{RSPv}\left(R(5)=0.91, r^{2}=\right.$ 0.82, $\mathrm{p}<0.05$ ) only in CNQX animals (Figure 14). We also observed a strong, positive relationship between PTLp and RSPv normalized colocalization $\left(R(5)=0.92, r^{2}=0.84, p\right.$ 
$<0.05$ ) that was found only in the CNQX group. These results could be interpreted as evidence of cortical coordination despite hippocampus inactivation, which would support SMC. However, since these relationships were absent in $14 \mathrm{~d}$ saline controls, they may alternatively represent a cortical compensatory mechanism that occurs only when the hippocampus is compromised: an interpretation more in line with Multiple Trace Theory (Goshen et al., 2011; Nadel \& Moscovitch, 1997).

Finally, we chose to perform structural equation modeling at 14d. This allowed us to directly compare the saline and CNQX correlation models to determine whether significant differences were produced by hippocampus inactivation. SEM was performed similarly to $2 \mathrm{~d}$, however the connections between $\mathrm{CA} 1 \rightarrow$ BLA $\rightarrow$ CEA were excluded due to signal degradation in the amygdala. Saline and CNQX Normalized Colocalization models were found to differ based on path coefficients $(\mathrm{p}<0.01)$ (Figure 17). Following hippocampus inactivation, the path coefficient from PTLP $\rightarrow$ RSPV was significantly stronger than in saline controls (CNQX: $1.34, \mathrm{SE}=0.22$; Saline: $-0.17, \mathrm{SE}=1.00)$. However, due to the large SE of path coefficient strength in saline animals, this path alone did not drive the difference between the models $(\mathrm{p}>0.05)$. Another contributing factor was a decrease in RSPV $\rightarrow$ ACC path strength in CNQX mice (CNQX: 0.27, SE $=$ 0.21 ; Saline: $1.14, \mathrm{SE}=0.44)$. Once again, the $\mathrm{SE}$ of the saline path was large enough to preclude this one connection from driving the model difference $(p>0.05)$. Because each significant path change did not independently drive the difference in model fit, we tested the paths in combination. We found that a model where both PTLP $\rightarrow$ RSPV and RSPV $\rightarrow$ ACC path coefficients were allowed to be freely estimated significantly differed from 
a model in which both of these path coefficients were fixed across groups $(\mathrm{p}<0.01)$. This suggests that changes in the path coefficients of both cortical projections drove the difference between the 14d Saline and CNQX models. In summary, hippocampus inactivation changed the overall patterns of cortical coordination at 14 days by inducing both a specific gain (PTLp $\rightarrow$ RSPv) and loss (RSPv $\rightarrow$ ACC) of cortical functional connectivity. This can be interpreted as evidence of cortical network reorganization resulting from hippocampus inactivation 14 days after training. 


\section{DISCUSSION}

In this chapter, we sought to experimentally verify a fundamental assumption of current models of memory: that the hippocampus is necessary for the reactivation of cortical neurons during memory retrieval. To do this, we tagged neocortical neurons with H2BGFP during context fear conditioning and examined whether these same neurons reactivate when the hippocampus is inactivated prior to retrieval 2 or 14 days later.

The data demonstrate the importance of the hippocampus to memory retrieval 2 days after training. First, we observed that inactivating CA1 prior to testing impaired $2 \mathrm{~d}$ memory retrieval. Second, we found an interesting and robust correlation between CA1 activity during learning (i.e. H2B-GFP tagging) and the strength of subsequent memory retrieval two days later. Third, we observed a strong, positive correlation between CA1 reactivation and freezing during the context test. This demonstrates that reducing the reactivation of CA1 neurons impairs memory retrieval soon after learning has occurred. This result is interesting considering that animals which froze less would have had the opportunity to explore more of context $\mathrm{A}$ and activate a potentially larger number of CA1 place cells. We observed the opposite effect, where increased CA1 reactivation correlated with increased freezing behavior (i.e. immobility). This relationship indicates that reactivation of $\mathrm{CA} 1$ neurons is more than a side product of re-exposure to the training environment, but is instead an important predictor of successful memory retrieval.

The data next demonstrate the importance of the hippocampus to cortical reactivation during recent memory retrieval. Hippocampus inactivation at two days reduced reactivation in the anterior cingulate and retrosplenial cortices, and eliminated patterns of 
correlated cortical activity observed in control animals during testing. These results represent the first direct evidence that the hippocampus is important for neocortical reactivation to occur during memory retrieval, and support memory models based on reactivation hypotheses (Alvarez \& Squire, 1994; Nadel \& Moscovitch, 1997; Winocur \& Moscovitch, 2011).

In contrast, hippocampus inactivation fourteen days after training did not significantly impair memory retrieval for the training context. This result suggests that the systems mediating memory retrieval reorganize in the weeks following training, and support our use of 14 days as a remote retrieval time point. In sharp contrast to inactivation at 2 days, inactivation 14 days after training did not result in impaired neocortical reactivation in the ACC or RSPv. At first glance, these results appear to support the Standard Model of Systems Consolidation, which predicts that neocortical reactivation should occur independently of the hippocampus following consolidation (Alvarez \& Squire, 1994). However, this interpretation of the data is weakened by the absence of a significant increase in the cortical reactivation of CNQX animals from 2 to 14 days. If SMC was directly supported by our data, then we would expect to see similar levels of cortical reactivation in $2 \mathrm{~d}$ saline, $14 \mathrm{~d}$ saline, and $14 \mathrm{~d}$ CNQX groups, with reactivation only decreased in 2d CNQX animals. Instead, we observed a numeric reduction in cortical reactivation from 2 to $14 \mathrm{~d}$ in saline mice. This reduction at $14 \mathrm{~d}$ may have driven the lack of impaired cortical reactivation following $14 \mathrm{~d}$ hippocampus inactivation. 
Additionally, SEM revealed that hippocampus inactivation at 2 days eliminated patterns of cortical coordination (PTLp $\rightarrow$ RSPv) observed in saline animals. We therefore predicted that if SMC was directly supported by our data, then we would observe 14-day hippocampus inactivation resulting in a sparing of the cortical correlations observed in control animals. Instead, we observed a lack of cortical correlations in the saline group and the emergence of CA1 $\rightarrow$ RSPv and PTLp $\rightarrow$ RSPv coordination only following hippocampus inactivation.

Together, these results may support a modified version of SMC where neocortical circuits are established after learning, but do not replace the original memory representations stored in the hippocampus (Winocur \& Moscovitch, 2011). As a result, hippocampus inactivation several weeks after training may induce a shift or reorganization in cortical coordination in an attempt to compensate for the loss of hippocampus input. Recent work by Deisseroth and colleagues (2011) supports cortical compensation following hippocampus inactivation at a remote time point. They demonstrate that prolonged (30 minute) pharmacological inactivation of CA1 results in an adaptive shift to the ACC during remote memory retrieval. This shift was absent when CA1 was rapidly silenced using optogenetics, which the authors assume did not provide a long enough temporal window for cortical compensation to occur.

Finally, we must acknowledge that our experiments have two major limitations. First, 14 days is not an optimal time point for testing recent vs. remote memory predictions of the SMC. Unfortunately, the stability of H2B-GFP expression outside of CA1 was limited to 
14 days after induction. This prevented us from examining the impact of hippocampus inactivation on cortical reactivation at a more traditional 28-day time point (Frankland \& Bontempi, 2005; Squire, 1992; Wiltgen, Brown, Talton, \& Silva, 2004). We may have observed different patterns of cortical coordination in both saline and CNQX groups had we been able to examine reactivation at 28 days. Future studies should take advantage of a newly developed tagging approach called TRAP: targeted recombination in active populations (Guenthner, Miyamichi, Yang, Heller, \& Luo, 2013). In these mice, activitydependent expression of a fluorescent effector gene is dependent on the presence of tamoxifen. When tamoxifen is administered prior to training, CreERT2 is expressed in active cells and induces excision of a loxP-flanked transcriptional stop signal. This results in ubiquitous expression of a fluorescent protein such as tdTomato. The advantage of this system is that the time window of activation is short $(<12 \mathrm{~h})$, background expression levels are very low, and activity-induced labeling is permanent.

Second, pharmacological inactivation of CA1 cannot specifically silence only those CA1 neurons that were engaged during learning. In contrast, optogenetic techniques can be used to control the activity of specific cell populations. Activation or inactivation is achieved via expression of light-sensitive proteins such as channelrhodopsin (ChR2), halorhodopsin (NpHR), or archaerhodopsin (Arch) (Yizhar, Fenno, Davidson, Mogri, \& Deisseroth, 2011). Recent work has shown that optogenetic methods can be combined with the tetracycline transactivator system to control the activity of hippocampal neurons that were engaged during learning (Liu et al., 2012). In future experiments, this powerful tool could be used to examine the contribution of previously activated hippocampal 
neurons to neocortical reactivation during recent and remote memory retrieval. 
A.

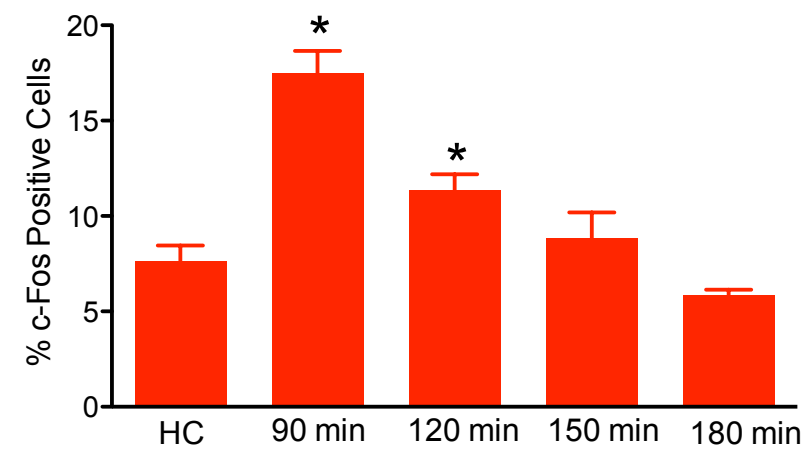

C. Behavioral Design

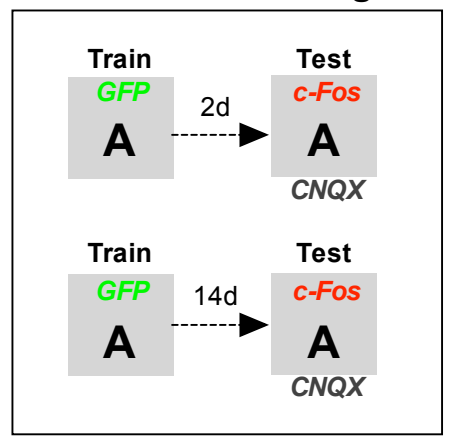

B.

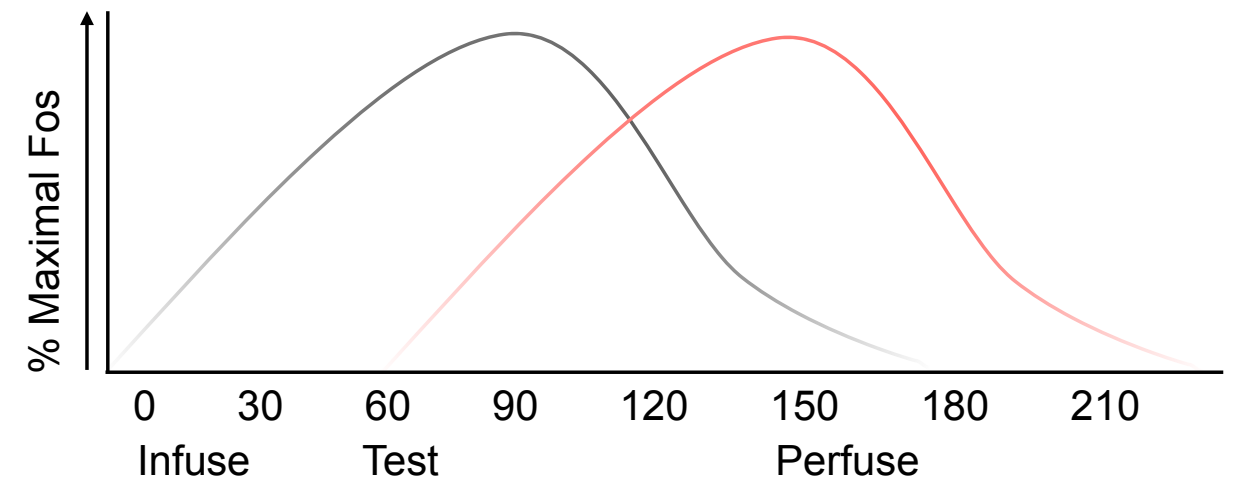

Figure 1. CA1 c-Fos expression time course and inactivation design. A) Expression of c-Fos reached a maximum $\%$ of positive neurons in CA1 ninety minutes following infusion handling and returned to homecage levels 150 minutes after handling. B) Mice were infused at time 0 , tested in context A 60 minutes after the infusion, and perfused 90 minutes later to restrict c-Fos expression to testing. C) Behavioral Design: mice were trained in context A off DOX to label activated neurons with H2B-GFP. Two or fourteen days later the hippocampus was inactivated using CNQX and mice were tested in the training context. 

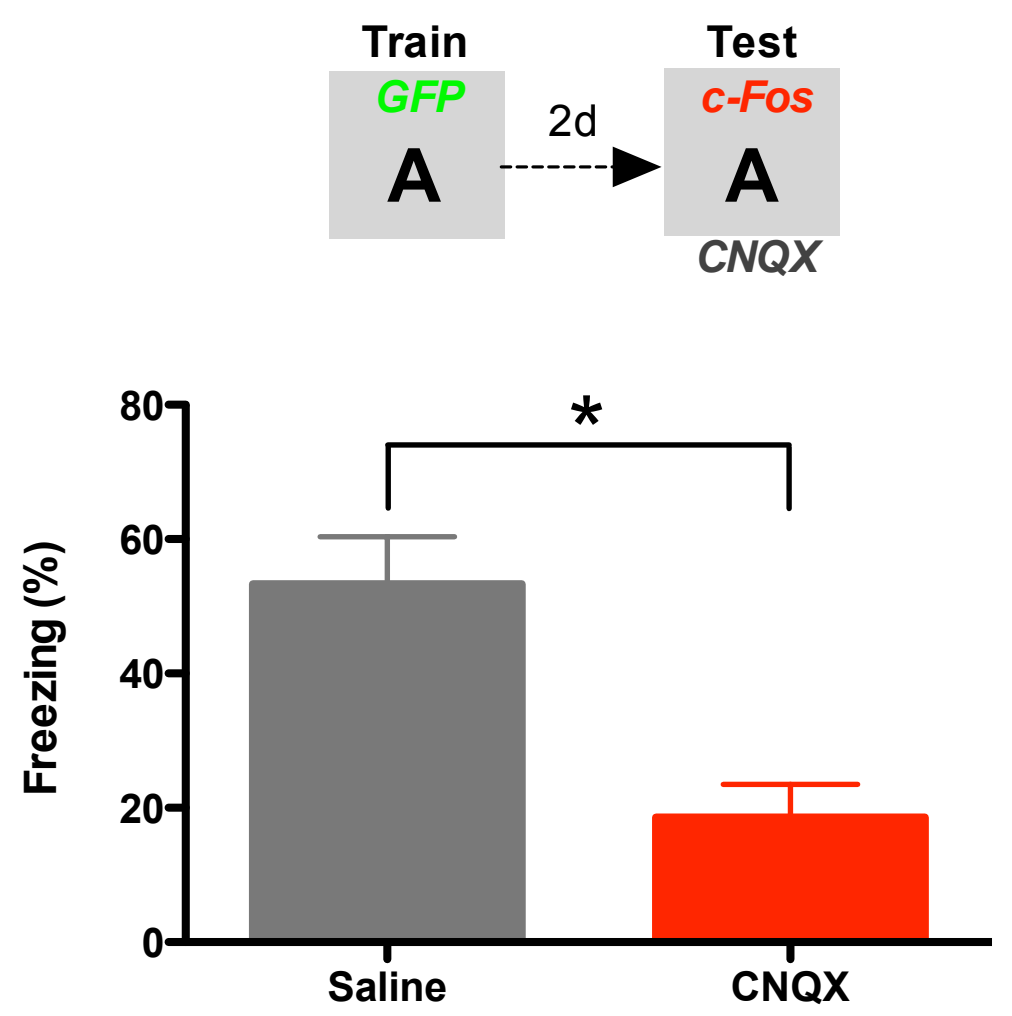

Figure 2. Hippocampus inactivation at $2 \mathrm{~d}$ impairs memory retrieval. TetTag mice were trained off DOX in context A and tested 2 days later (Saline $n=7$, CNQX $n=7$ ). Infusion of CNQX into the dorsal hippocampus 60 minutes prior to testing impaired memory retrieval for context A. Error bars represent \pm SEM. $* p<0.05$. 
A.

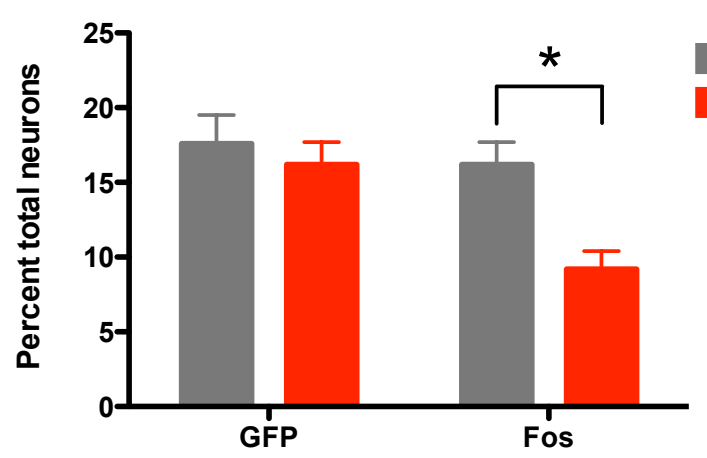

B.

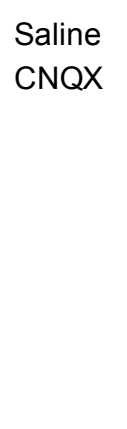

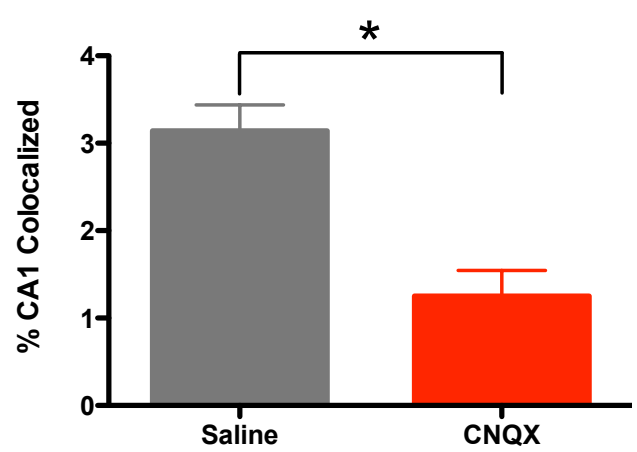

Figure 3. Hippocampus inactivation at $2 \mathrm{~d}$ impairs CA1 reactivation. A) Infusion of CNQX into the dorsal hippocampus 60 minutes prior to testing reduced the expression of c-Fos in CA1. H2B-GFP expression (which occurred during context training) was unchanged. B) CNQX inactivation impaired the reactivation of neurons in CA1. Error bars represent \pm SEM. ${ }^{*} \mathrm{p}<0.05$. 


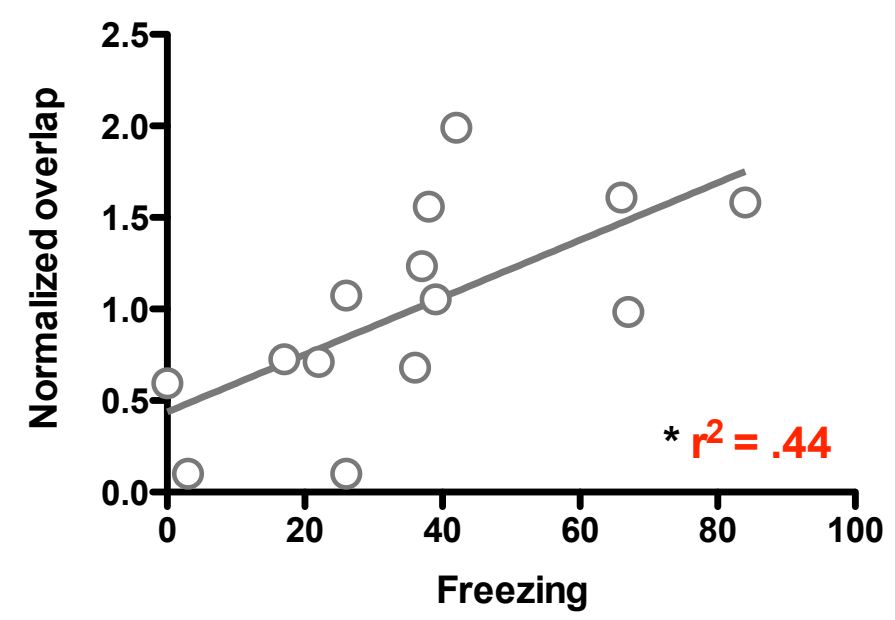

Figure 4. Hippocampus reactivation correlates with the strength of $2 \mathrm{~d}$ memory retrieval. The percentage of reactivated CA1 neurons correlated significantly with freezing behavior at testing. ${ }^{*} \mathrm{p}<0.05$. 
A.

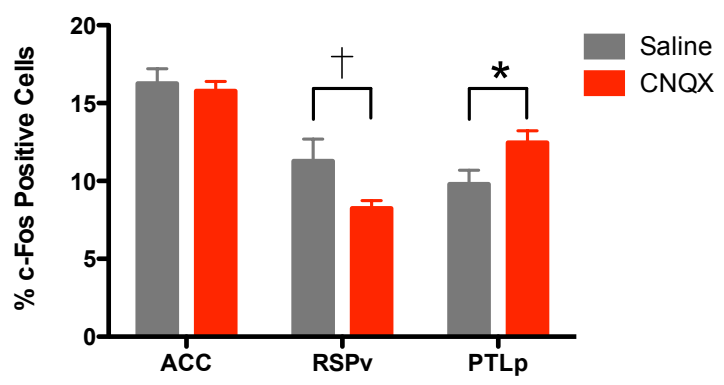

B.

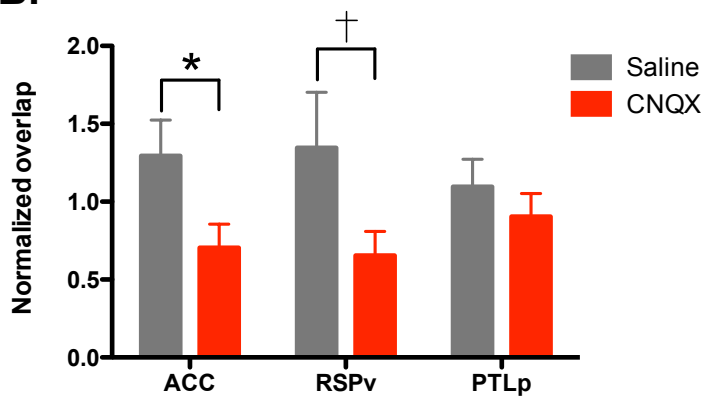

Figure 5. The hippocampus is necessary for the reactivation of neocortical neurons

at 2d. A) Hippocampus inactivation did not impair c-Fos expression in the ACC or PTLp, but did cause a strong trend towards reduction in the RSPv. B) Hippocampus inactivation reduced reactivation in the ACC and caused a strong trend towards reduction in the RSPv, but did not affect the PTLp. The percentage of double-labeled neurons is shown normalized to the average percentage of overlap in each brain region for all mice. Error bars represent \pm SEM. ${ }^{*} \mathrm{p}<0.05 . \dagger p<0.1$ 

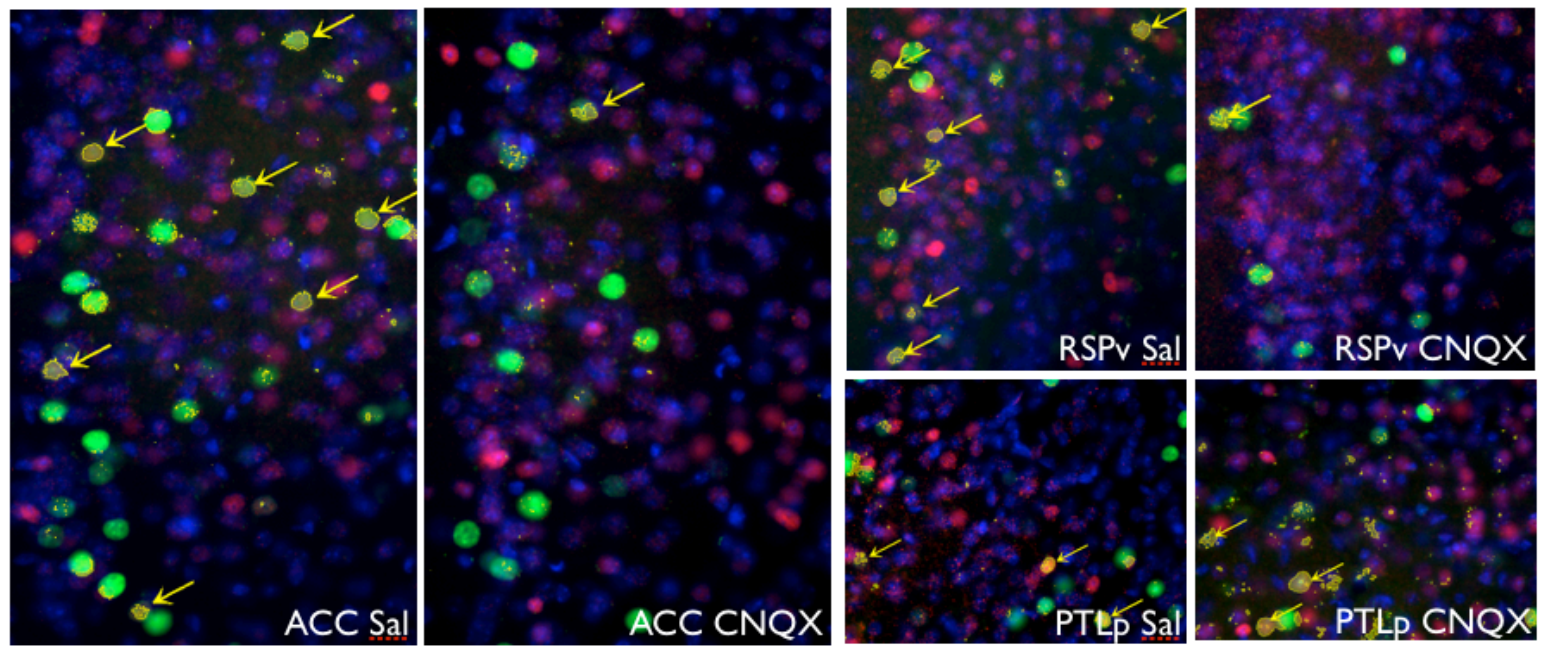

Figure 6. Hippocampus inactivation at two days impairs the reactivation of neocortical neurons. Hippocampus inactivation (right in each panel) reduced the reactivation of ACC and RSPv, but not PTLp H2B-GFP neurons tagged during learning. H2B-GFP shown in green, c-Fos in red, DAPI counterstaining in blue. Arrows indicate reactivated neurons. 


\section{2d GFP Correlations}
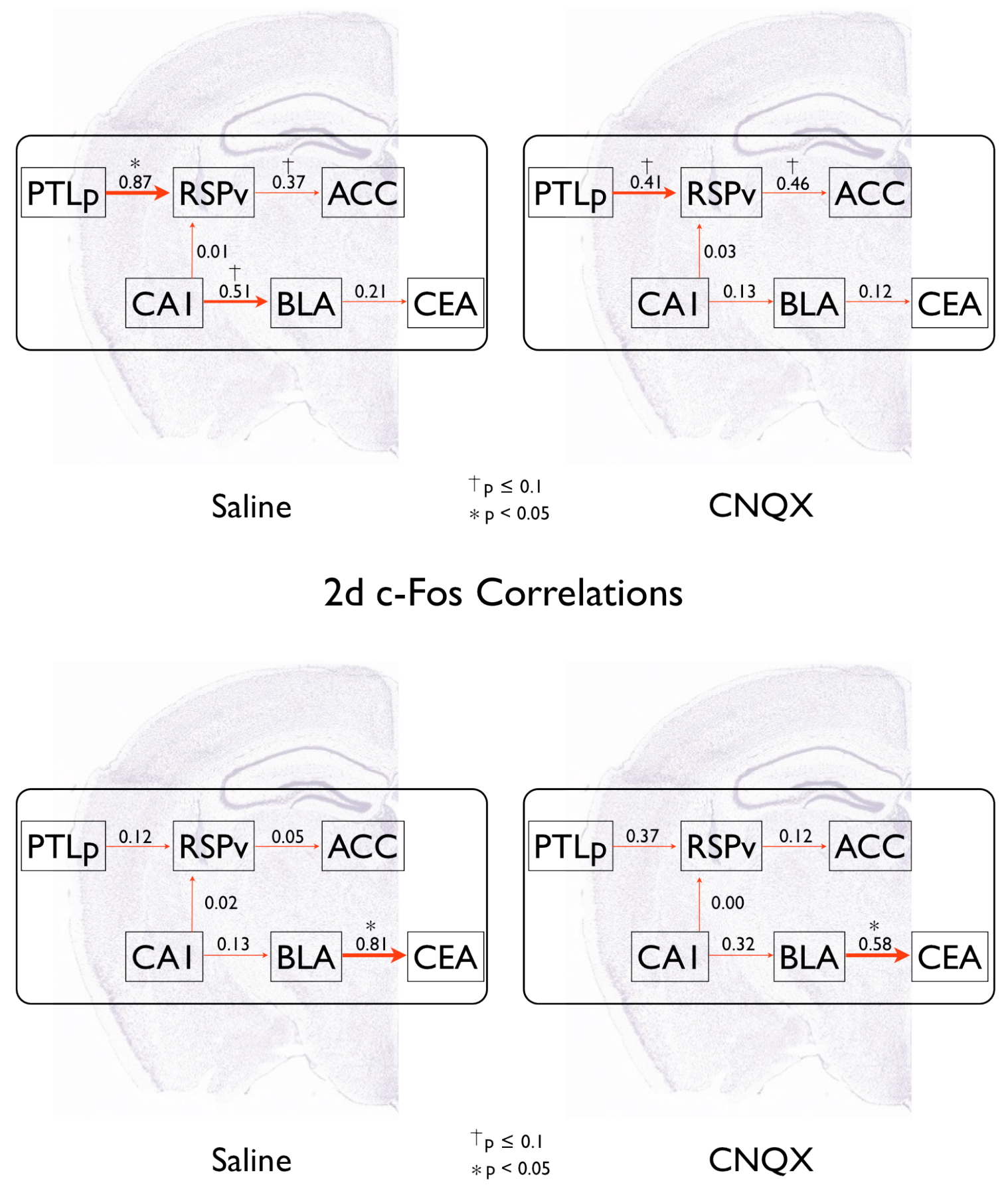

Figure 7. Hippocampus inactivation did not impair H2B-GFP and c-Fos regional correlations. As expected, regional correlations ( $\mathrm{r}^{2}$ scores) for training-induced GFP activity (Top) were similar in CNQX $(n=7)$ and saline $(n=7)$ animals. Both groups also 
exhibited strong, positive correlations for c-Fos activity between the BLA and CEA

(Bottom). Data shown as $\mathrm{r}^{2}$ scores. ${ }^{*} \mathrm{p}<0.05 . \dagger \mathrm{p}<0.1$ 


\section{2d Normalized Colocalization Correlations}

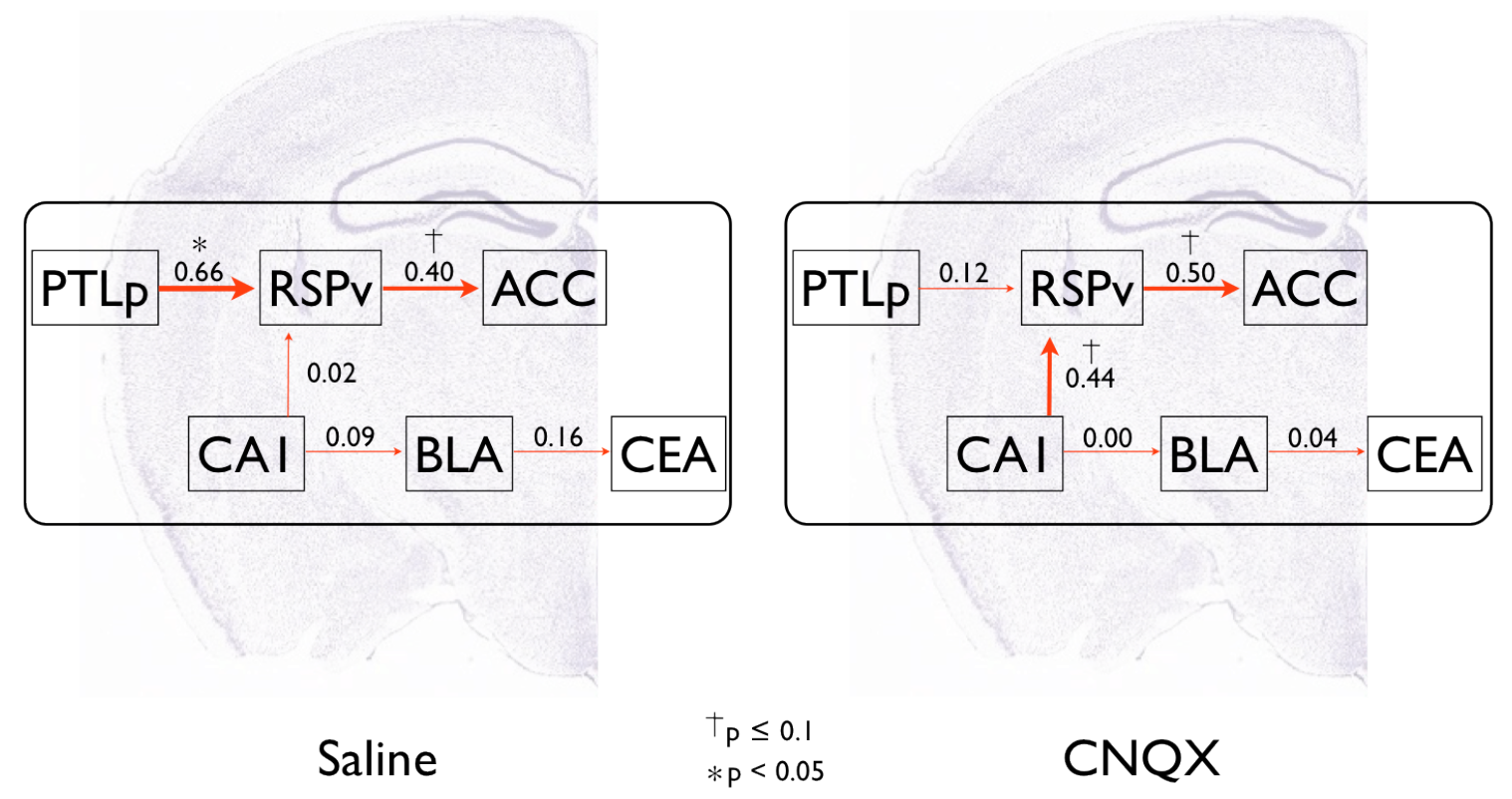

Figure 8. Hippocampus inactivation impairs correlation between the PTLp and RSPv during $2 d$ retrieval. The degree of reactivation (i.e. colocalization normalized to the regional mean for all mice) was positively correlated in saline animals between the PTLp and RSPv during memory retrieval. This cortical correlation was eliminated by hippocampus CNQX inactivation. Data shown as $\mathrm{r}^{2}$ scores. ${ }^{*} \mathrm{p}<0.05 . \dagger \mathrm{p}<0.1$ 
2D Saline
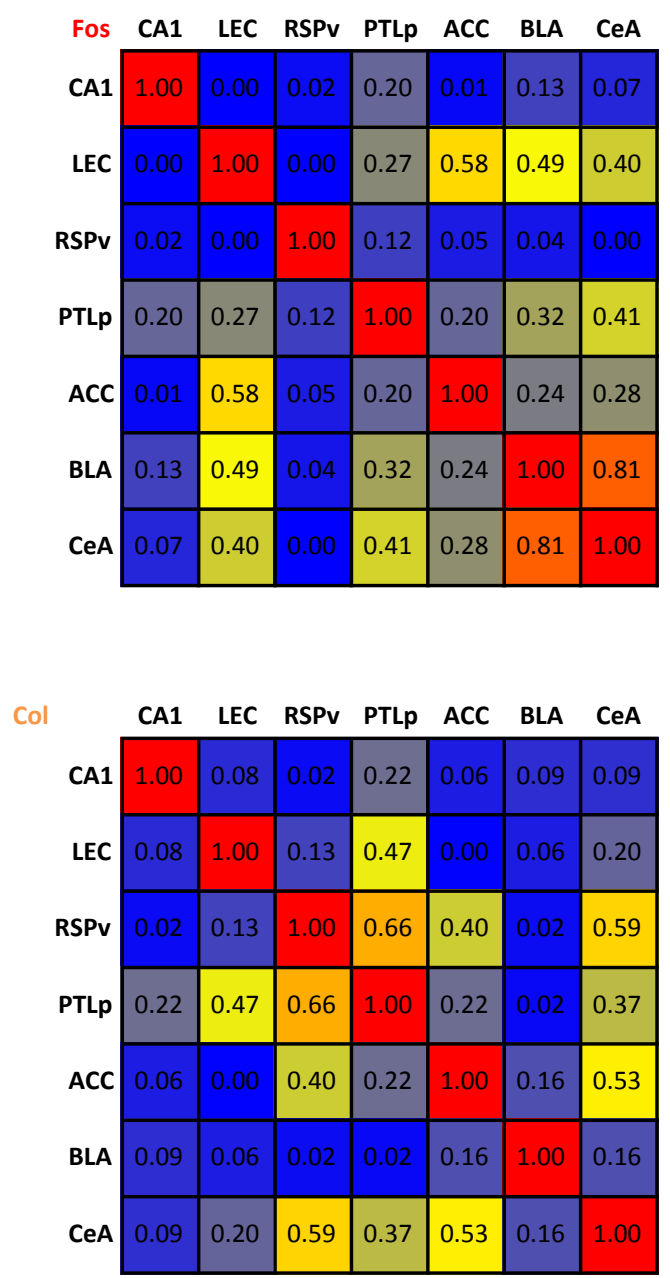

2D CNQX
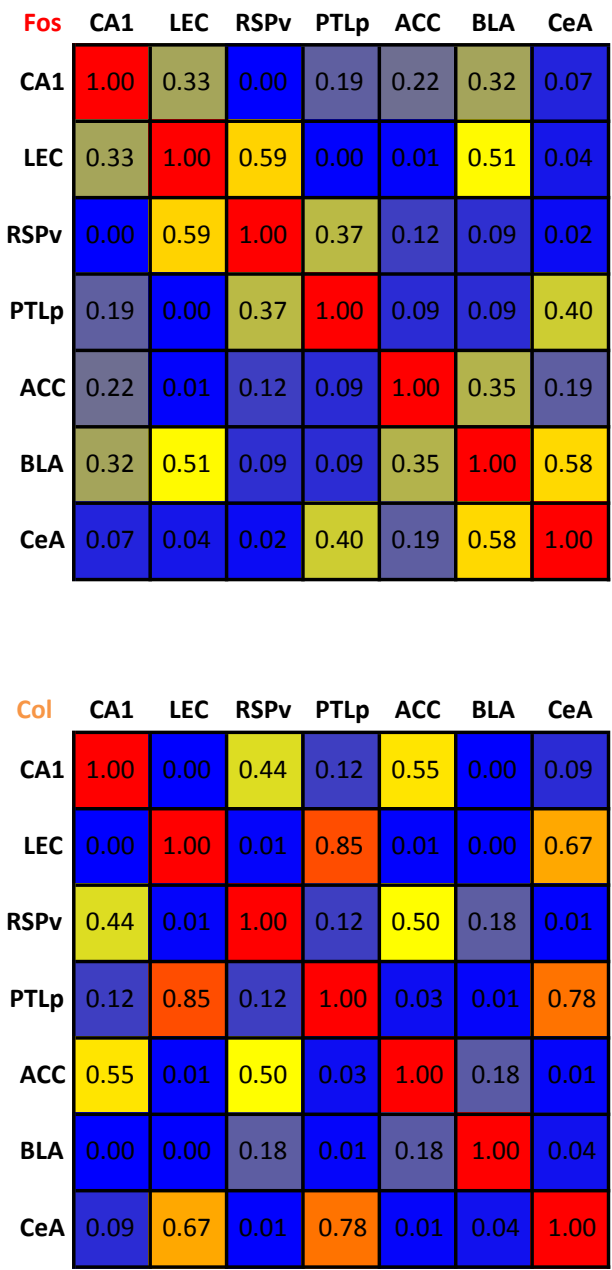

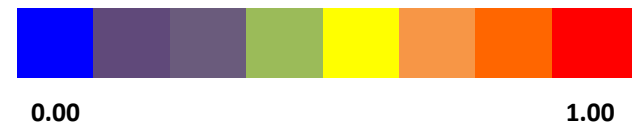

1.00

Figure 9. Correlation matrices examining immediate early gene and reactivation

activity during $\mathbf{2 d}$ memory retrieval. Saline controls (top left) and inactivated animals (top right) exhibited positive correlations in c-Fos activity between the BLA and CEA during memory retrieval. Normalized colocalization was also positively correlated between the RSPv and PTLp during memory retrieval (bottom left). This cortical correlation was absent in inactivated mice (bottom right). Data shown as $\mathrm{r}^{2}$ scores. 


\section{2d Normalized Colocalization SEM}

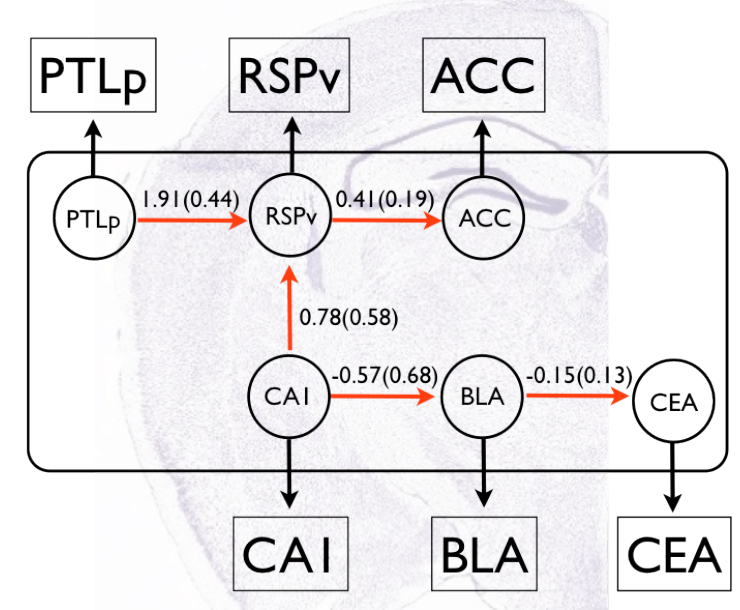

Saline

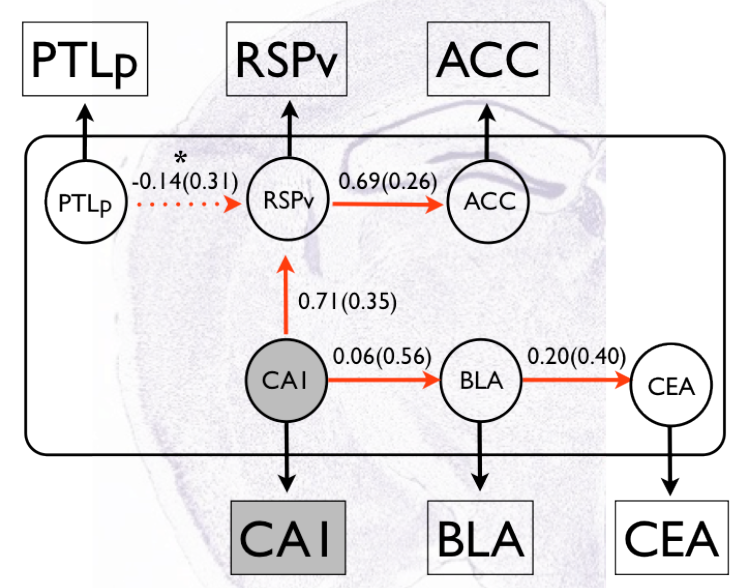

CNQX

Figure 10. Impact of hippocampus inactivation on functional connectivity models at

2d. Functional connectivity was examined using structural equation modeling (SEM).

Changes in functional connectivity were estimated between hippocampal, cortical, and amygdala regions linked by known anatomical pathways. Saline (left) and CNQX (right) models generated using normalized colocalization data differed based on path coefficients. A decrease in path strength between PTLP $\rightarrow$ RSPV in CNQX animals drove the difference in the models. Path coefficients (Standard Error in parentheses) reflect the estimate and direction of covariance between brain regions. ${ }^{*} \mathrm{p}<0.05$. 


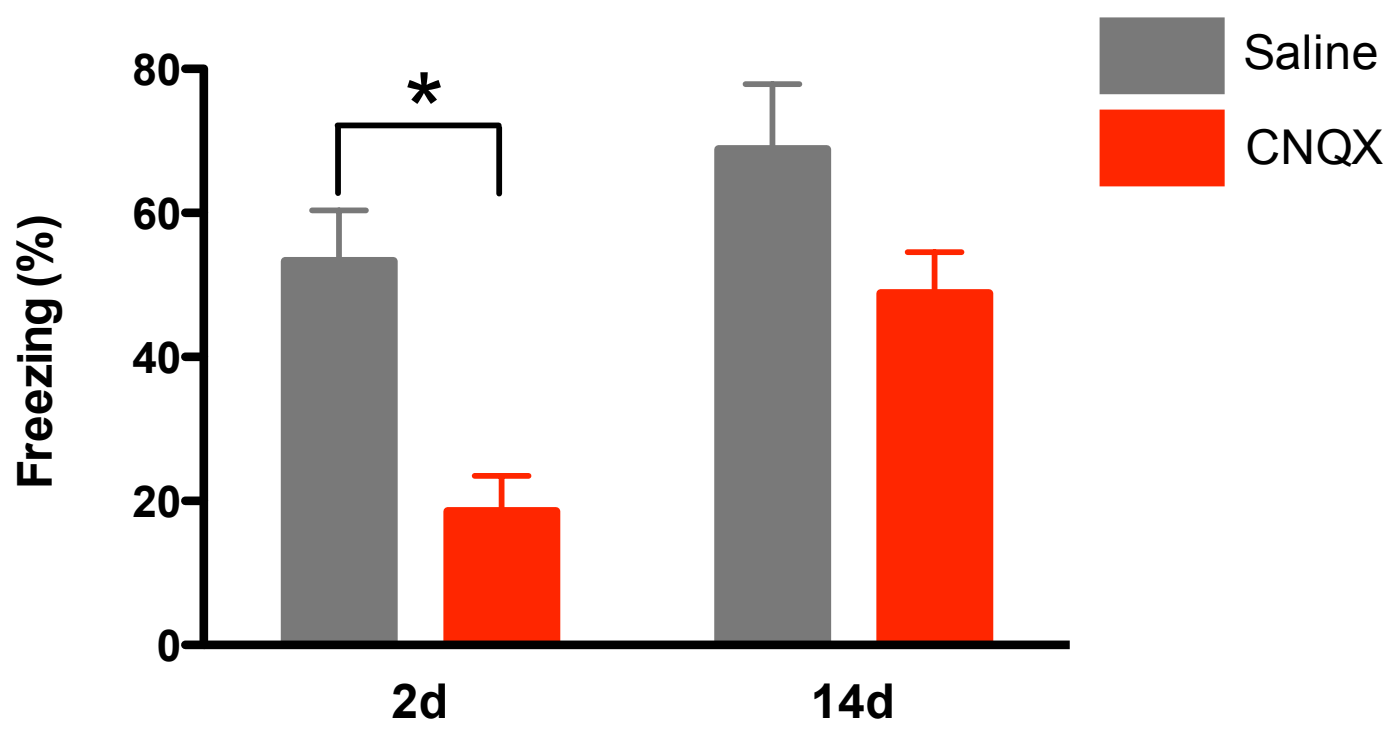

Figure 11. Hippocampus inactivation at $14 \mathrm{~d}$ did not impair memory retrieval.

TetTag mice were trained off DOX in context A and tested 2 or 14 days later (2d: Saline $\mathrm{n}=7, \mathrm{CNQX} \mathrm{n}=7 ;$ 14d: Saline $\mathrm{n}=6, \mathrm{CNQX} \mathrm{n}=7$ ). Infusion of CNQX into the dorsal hippocampus 60 minutes prior to testing failed to impair memory retrieval for context $\mathrm{A}$ at 14 days. Error bars represent \pm SEM. ${ }^{*} \mathrm{p}<0.05$. 
A.
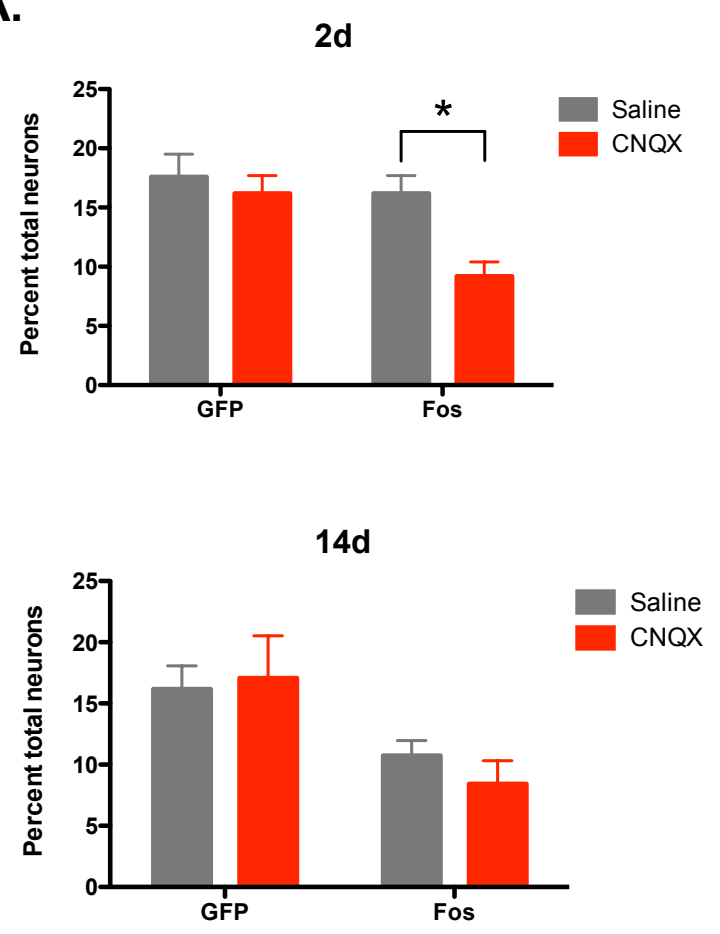

B.
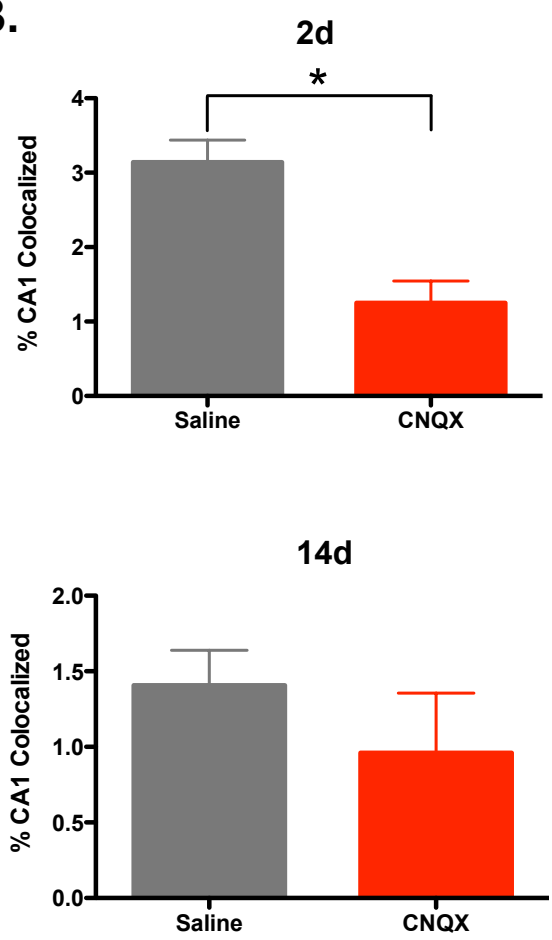

Figure 12. Impact of hippocampus inactivation on CA1 c-Fos expression and reactivation at 14d. A) Top: Infusion of CNQX into the dorsal hippocampus 60 minutes prior to testing at 2 days reduced the expression of c-Fos in CA1. Bottom: Inactivation at $14 \mathrm{~d}$ produced a numeric decrease in CA1 c-Fos expression, but this result was not significant. B) Top: CNQX inactivation impaired the reactivation of neurons in CA1 at 2 days. Bottom: Inactivation at $14 \mathrm{~d}$ produced a numeric decrease in CA1 reactivation, but this result was not significant. Error bars represent \pm SEM. ${ }^{*} p<0.05$. 
A.

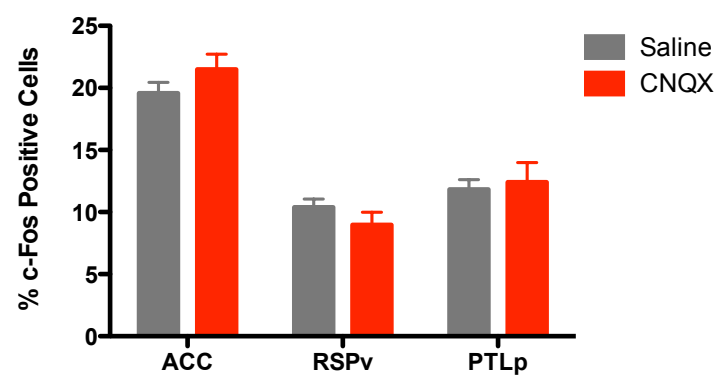

B.

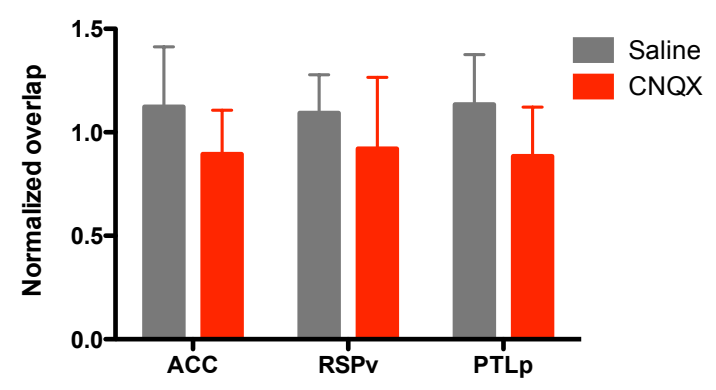

Figure 13. Hippocampus inactivation does not impair neocortical reactivation at

14d. A) Hippocampus inactivation did not impair c-Fos expression in the ACC, RSPv, or PTLp. B) Hippocampus inactivation did not impair reactivation in the ACC, RSPv, or PTLp. The percentage of double-labeled neurons is shown normalized to the average percentage of overlap in each brain region for all mice. Error bars represent \pm SEM. ${ }^{*} p<$ 0.05 . 


\section{I4d GFP Correlations}

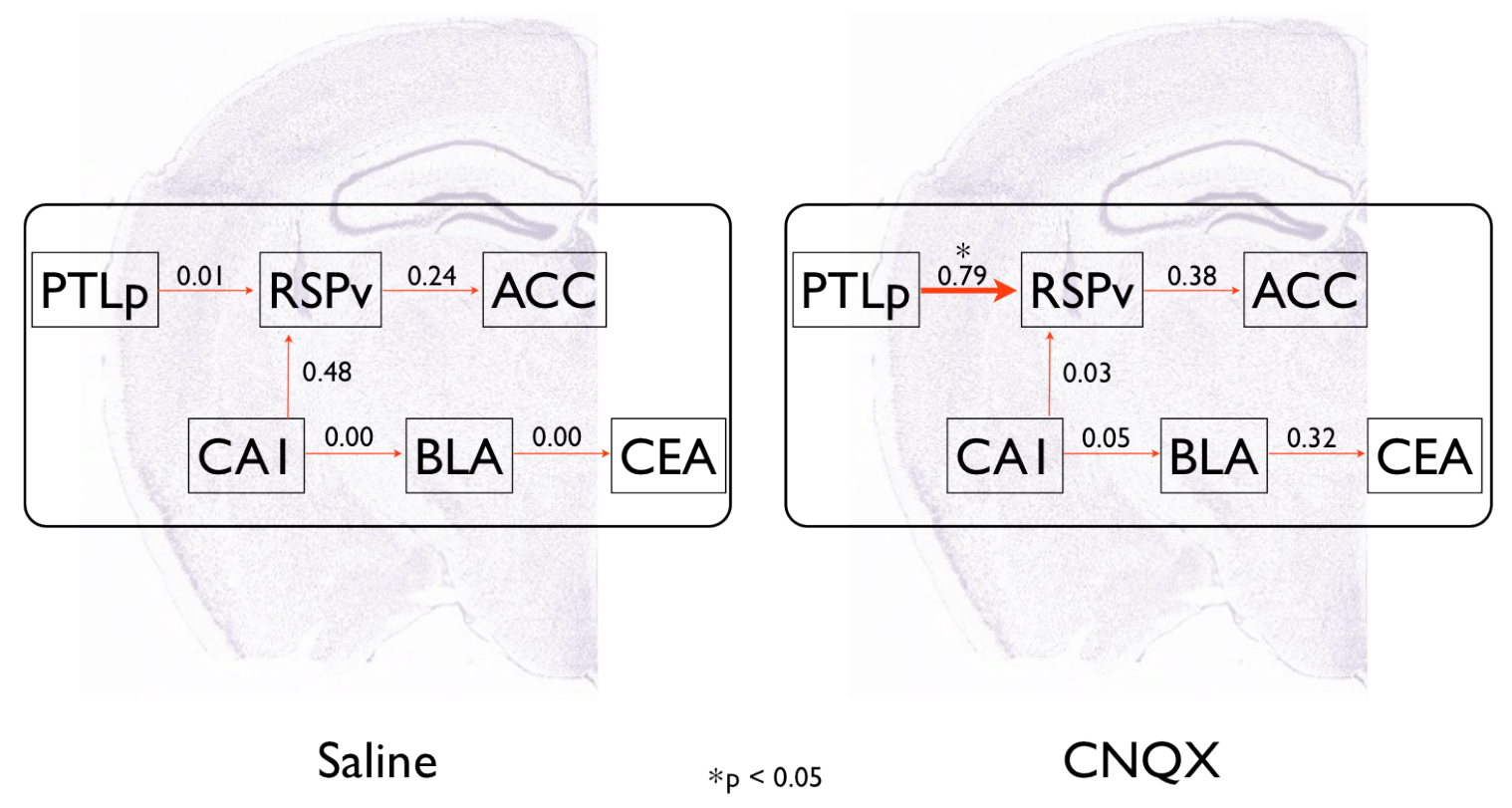

\section{I4d c-Fos Correlations}
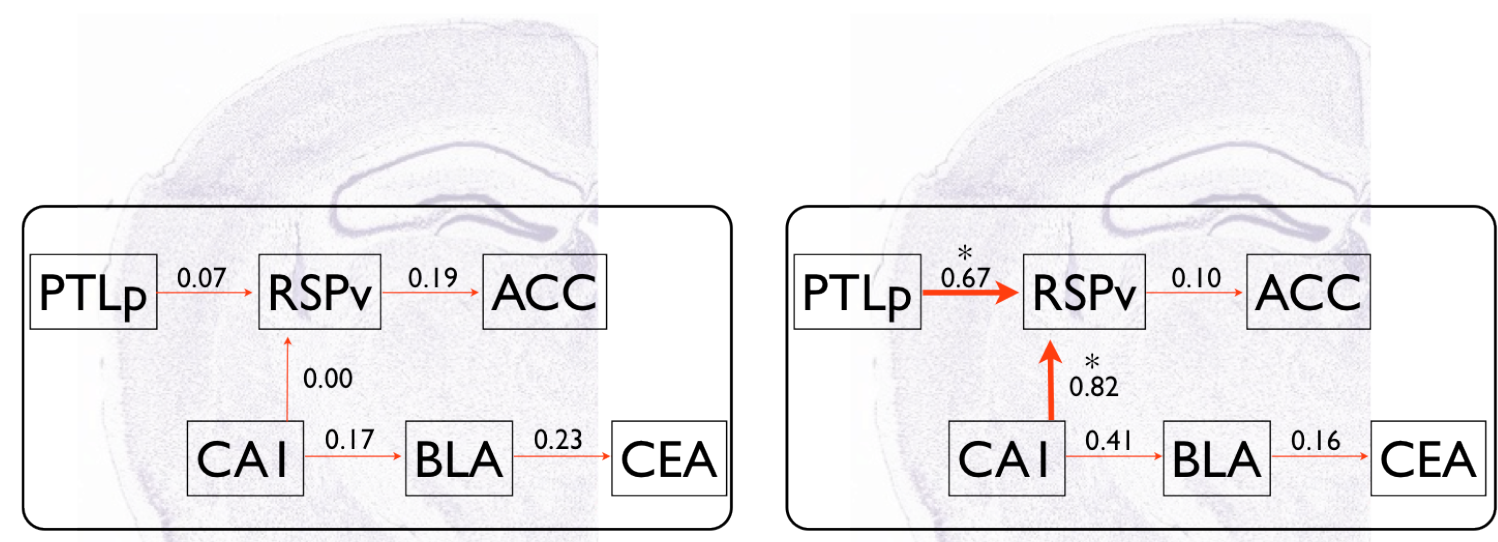

Saline

$*_{p}<0.05$

CNQX

Figure 14. Hippocampus inactivation at 14d induces reorganization of c-Fos

regional correlations. Top: H2B-GFP correlations $\left(\mathrm{r}^{2}\right.$ scores $)$ were similar across saline 
$(n=6)$ and CNQX $(n=7)$ groups. Bottom: CNQX animals alone demonstrated correlated c-Fos activity between PTLp and RSPv and between CA1 and RSPv. Data shown as $\mathrm{r}^{2}$ scores. ${ }^{*} \mathrm{p}<0.05$. 


\section{I4d Normalized Colocalization Correlations}

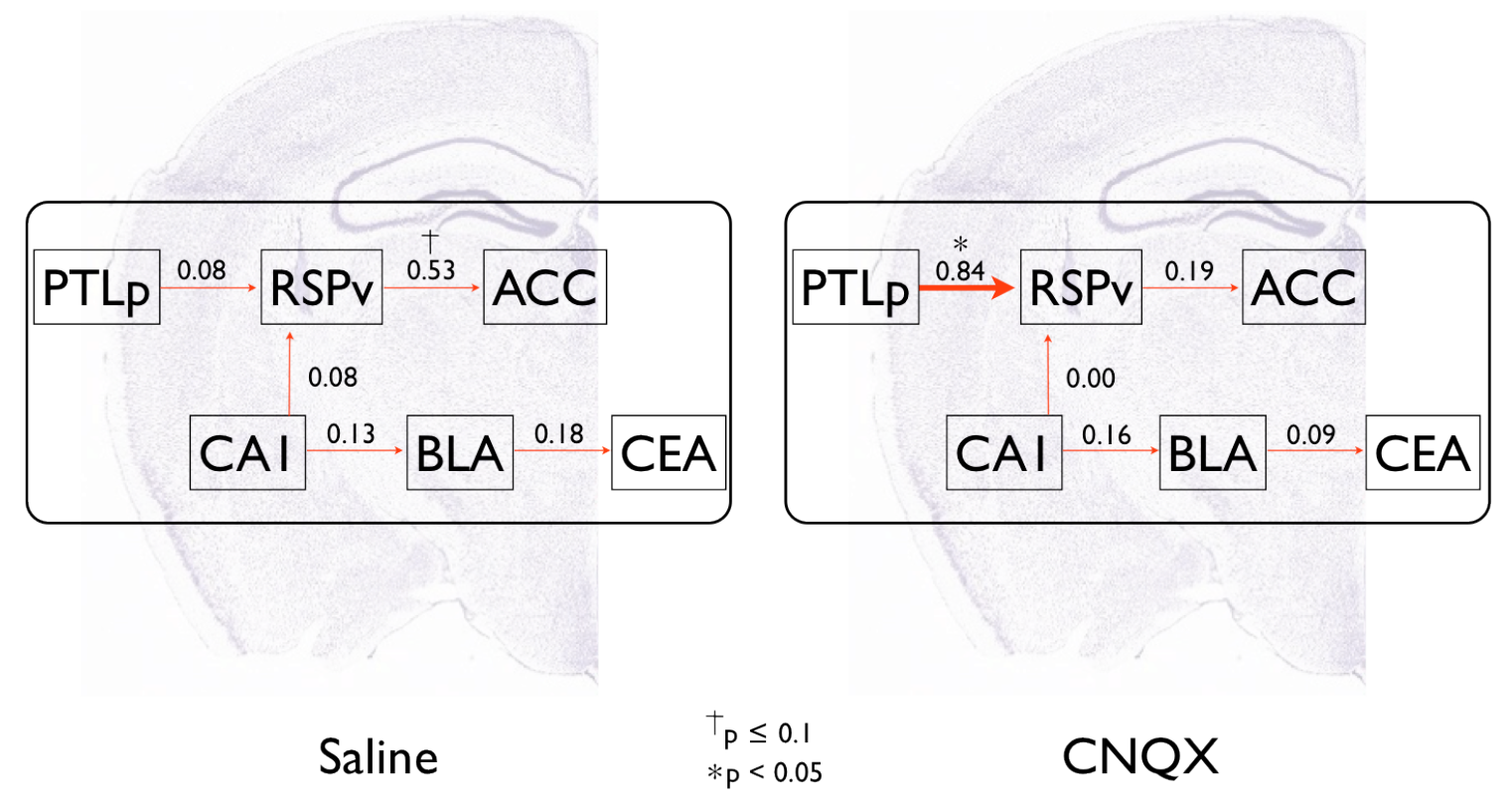

Figure 15. Hippocampus inactivation at $14 \mathrm{~d}$ results in the reorganization of cortical coordination during memory retrieval. Saline mice exhibited a strong trend towards correlation between the RSPv and ACC that was eliminated following hippocampus inactivation. However, a strong positive relationship also emerged between PTLp and RSPv following hippocampus inactivation. Data shown as $\mathrm{r}^{2}$ scores. ${ }^{*} \mathrm{p}<0.05 . \dagger \mathrm{p}<0.1$ 
14d Saline
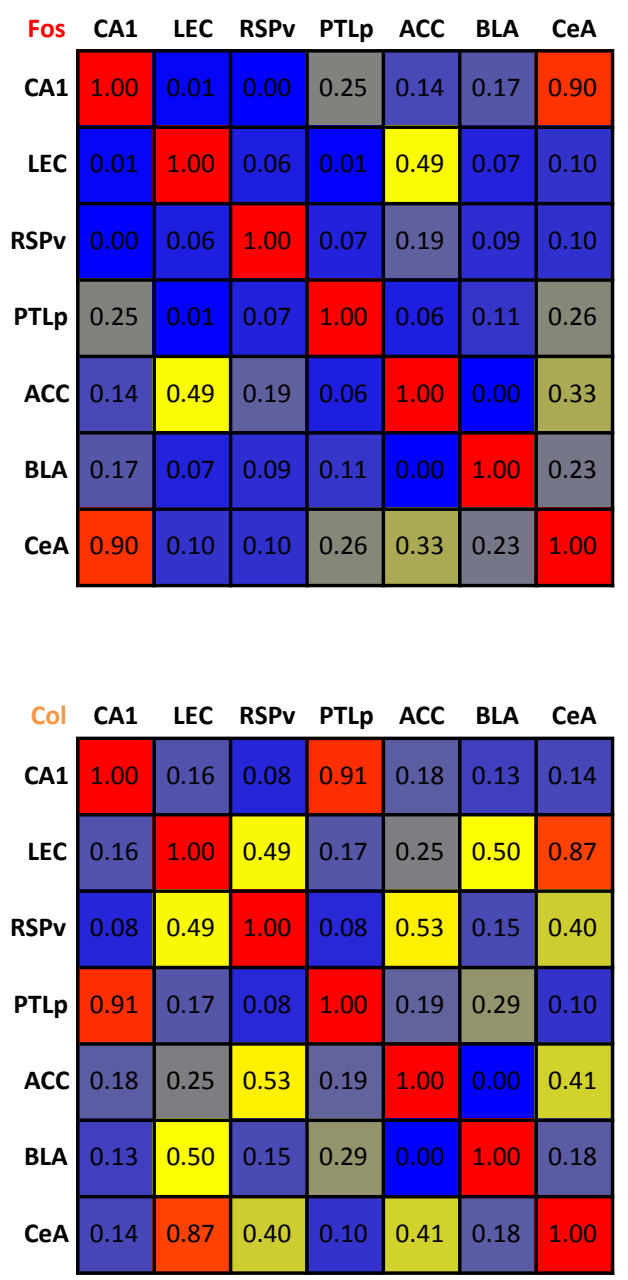

14d CNQX
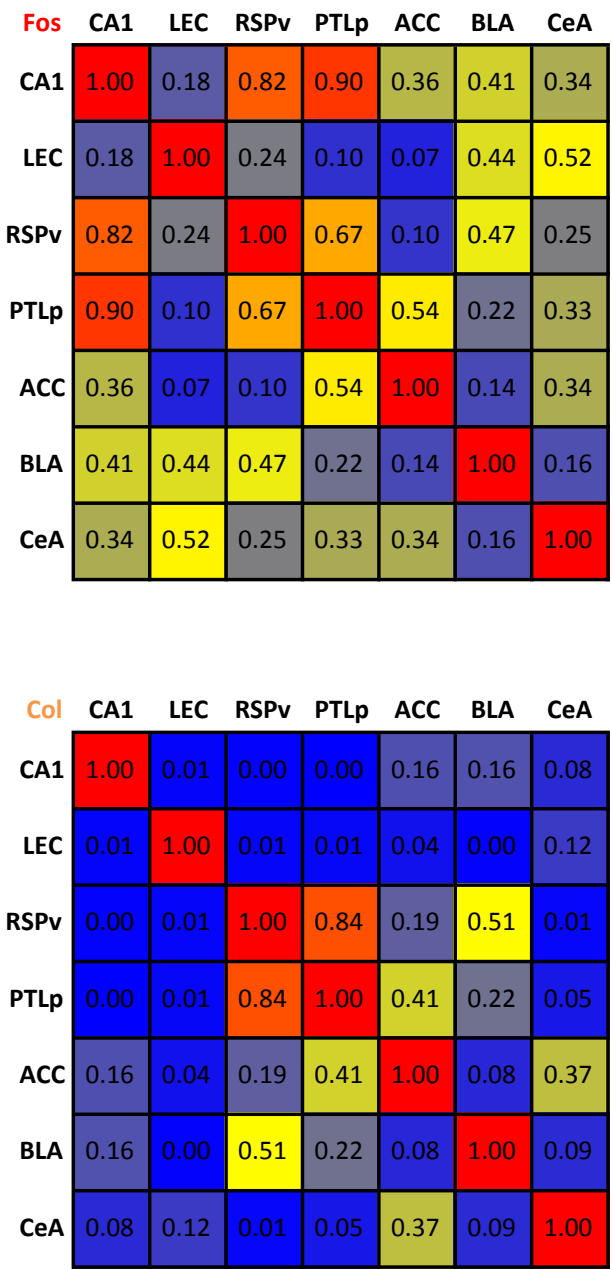

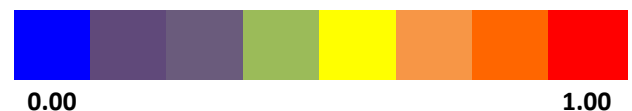

Figure 16. Correlation matrices examining immediate early gene and reactivation

activity during 14d memory retrieval. Inactivated animals (top right) exhibited positive correlations in c-Fos activity between CA1 and RSPv, and between PTLp and RSPv that were absent in saline controls. Normalized colocalization was also positively correlated between PTLp and RSPv in CNQX animals (bottom right), but not saline controls (bottom left). However, saline animals did exhibit a strong trend towards correlation between the RSPv and ACC. Data shown as $\mathrm{r}^{2}$ scores. 


\section{I4d Normalized Colocalization SEM}

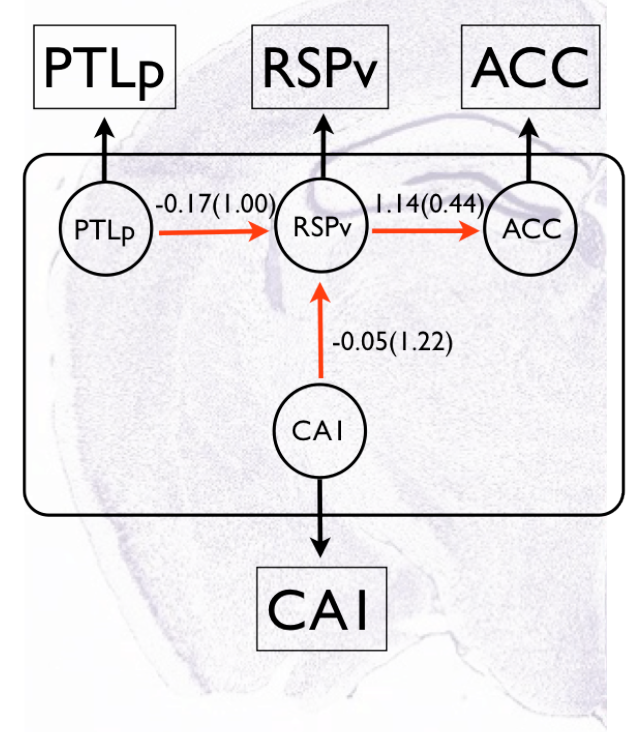

Saline

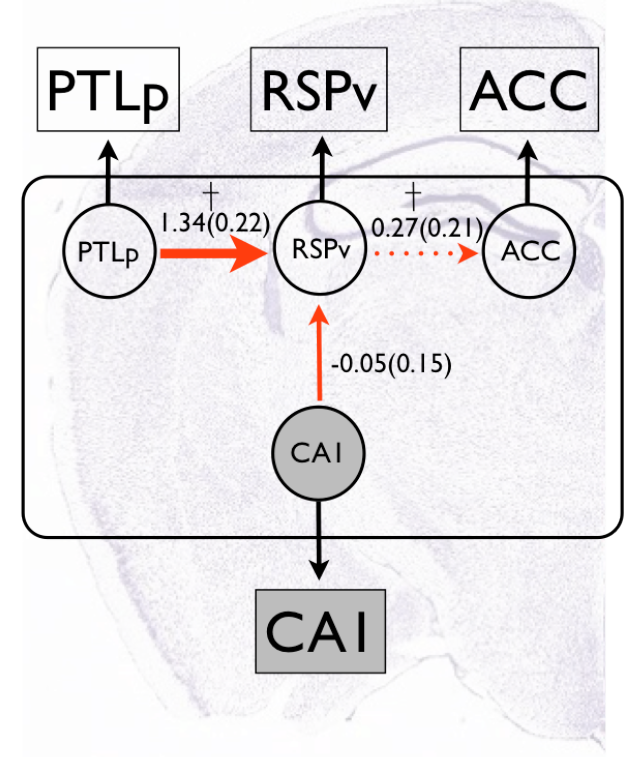

CNQX

Figure 17. Hippocampus inactivation at $14 d$ results in the reorganization of cortical functional connectivity during memory retrieval. Functional connectivity was examined using structural equation modeling (SEM). Changes in functional connectivity were estimated between hippocampal and cortical regions linked by known anatomical pathways. Saline (left) and CNQX (right) models generated using normalized colocalization data differed based on path coefficients. Changes in the path weights of both PTLP $\rightarrow$ RSPV and RSPv $\rightarrow$ ACC drove the difference in the models. Path coefficients (Standard Error in parentheses) reflect the estimate and direction of covariance between brain regions. ${ }^{*} \mathrm{p}<0.05 . \dagger \mathrm{p}<0.1$ 


\section{REFERENCES}

Addis, D. R., \& McAndrews, M. P. (2006). Prefrontal and hippocampal contributions to the generation and binding of semantic associations during successful encoding. NeuroImage, 33(4), 1194-1206.

Alvarez, P., \& Squire, L. R. (1994). Memory consolidation and the medial temporal lobe: a simple network model. Proc Natl Acad Sci U S A, 91(15), 7041-7045.

Andersen, P., Morris, R., Amaral, D., Bliss, T., O’Keefe, J. (2007). The Hippocampus Book. New York, NY: Oxford University Press.

Boker, S. M., Neale, M. C., Maes, H. H., Wilde, M. J., Spiegel, M., Brick, T. R., . . Brandmaier, A. (2012). OpenMx 1.2 User Guide.

Boker, S. M., Neale, M. C., Maes, H. H., Wilde, M. J., Spiegel, M., Brick, T. R., . . Fox, J. (2011). OpenMx: An Open Source Extended Structural Equation Modeling Framework. Psychometrika.

Cenquizca, L. A., \& Swanson, L. W. (2007). Spatial organization of direct hippocampal field CA1 axonal projections to the rest of the cerebral cortex. Brain Research Reviews, 56(1), 1-26.

Dong, H. W. (2008). The Allen Reference Atlas. Hoboken, NJ: Wiley.

Frankland, P. W., \& Bontempi, B. (2005). The organization of recent and remote memories. Nat Rev Neurosci, 6(2), 119-130.

Frankland, P. W., Bontempi, B., Talton, L. E., Kaczmarek, L., \& Silva, A. J. (2004). The Involvement of the Anterior Cingulate Cortex in Remote Contextual Fear Memory. Science, 304(5672), 881-883. 
Goshen, I., Brodsky, M., Prakash, R., Wallace, J., Gradinaru, V., Ramakrishnan, C., \& Deisseroth, K. (2011). Dynamics of Retrieval Strategies for Remote Memories. Cell, 147(3), 678-689.

Guenthner, C., Miyamichi, K., Yang, H., Heller, H., \& Luo, L. (2013). Permanent genetic access to transiently active neurons via TRAP: Targeted recombination in active populations. Neuron(78), 773-784.

Hoffman, K. L., \& McNaughton, B. L. (2002). Coordinated reactivation of distributed memory traces in primate neocortex. Science, 297(5589), 2070-2073.

Kesner, R. P. (2009). The posterior parietal cortex and long-term memory representation of spatial information. Neurobiology of Learning and Memory, 91(2), 197-206.

Kubik, S., Miyashita, T., Kubik-Zahorodna, A., \& Guzowski, J. (2012). Loss of activitydependent Arc gene expression in the retrosplenial cortex after hippocampal inactivation: interaction in a higher-order memory circuit. Neurobiology of Learning and Memory, Jan;97(1), 124-131.

Liu, X., Ramirez, S., Pang, P. T., Puryear, C. B., Govindarajan, A. F., Deisseroth, K., \& Tonegawa, S. (2012). Optogenetic stimulation of a hippocampal engram activates fear memory recall. Nature, 484(19 April ), 381-387.

Marr, D. (1971). Simple memory: a theory for archicortex. Philosophical transactions of the Royal Society of London. Series B, Biological sciences, 262(841), 23-81.

Maviel, T., Durkin, T. P., Menzaghi, F., \& Bontempi, B. (2004). Sites of Neocortical Reorganization Critical for Remote Spatial Memory. Science, 305(5680), 96-99.

McClelland, J. L., McNaughton, B. L., \& O'Reilly, R. C. (1995). Why there are complementary learning systems in the hippocampus and neocortex: insights from 
the successes and failures of connectionist models of learning and memory. Psychol Rev, 102(3), 419-457.

McIntosh, A. R., \& Gonzalez-Lima, F. (1994). Structural Equation Modeling and its Application to Network Analysis in Functional Brain Imaging. Human Brain Mapping(2), 2-22.

Moscovitch, M., Rosenbaum, R. S., Gilboa, A., Addis, D. R., Westmacott, R., Grady, C., ... Nadel, L. (2005). Functional neuroanatomy of remote episodic, semantic and spatial memory: a unified account based on multiple trace theory. J. Anat., 207, $35-66$.

Nadel, L., \& Moscovitch, M. (1997). Memory consolidation, retrograde amnesia and the hippocampal complex. Curr Opin Neurobiol, 7(2), 217-227.

O'Reilly, C., \& Rudy, J. W. (2001). Conjunctive Representations in Learning and Memory: Principles of Cortical and Hippocampal Function. Psychological Review, 108(2), 311-345.

Qin, Y., McNaughton, B., Skaggs, W., \& Barnes, C. A. (1997). Memory reprocessing in corticocortical and hippocampocortical neuronal ensembles. Philos Trans R Soc Lond B Biol Sci, 352(1360).

Siapas, A. G., \& Wilson, M. A. (1998). Coordinated Interactions between Hippocampal Ripples and Cortical Spindles during Slow-Wave Sleep. Neuron, 21(5), 11231128.

Squire, L. R. (1992). Memory and the hippocampus: a synthesis from findings with rats, monkeys, and humans. Psychol Rev, 99(2), 195-231. 
Tayler, K. K., Lowry, E., Tanaka, K., Levy, B., Reijmers, L., Mayford, M., \& Wiltgen, B. J. (2011). Characterization of NMDAR-Independent Learning in the Hippocampus. Front Behav Neurosci, 5, 28.

Tayler, K. K., Tanaka, K. Z., Reijmers, L. G., \& Wiltgen, B. J. (2013). Reactivation of Neural Ensembles during the Retrieval of Recent and Remote Memory. Curr Biol, 23(2).

Teyler, T. J., \& DiScenna, P. (1986). The hippocampal memory indexing theory. Behav Neurosci., Apr(100(2)), 147-154.

Teyler, T. J., \& Rudy, J. W. (2007). The hippocampal indexing theory and episodic memory: updating the index. [Review]. Hippocampus, 17(12), 1158-1169.

Vetere, G., Restivo, L., Cole, C. J., Ross, P. J., Ammassari-Teule, M., Josselyn, S. A., \& Frankland, P. W. (2011). Spine growth in the anterior cingulate cortex is necessary for the consolidation of contextual fear memory. Proc Natl Acad Sci U $S A, 108(20), 8456-8460$.

Wilson, M. A., \& McNaughton, B. L. (1994). Reactivation of Hippocampal Ensemble Memories During Sleep. Science, 265(5172), 676-679.

Wiltgen, B. J., Brown, R. A., Talton, L. E., \& Silva, A. J. (2004). New circuits for old memories: the role of the neocortex in consolidation. Neuron, 44(1), 101-108.

Winocur, G., \& Moscovitch, M. (2011). Memory transformation and systems consolidation. Journal of the International Neuropsychological Society, 17(5), 766-780. 
Winters, B. D., \& Bussey, T. J. (2005). Transient Inactivation of Perirhinal Cortex Disrupts Encoding, Retrieval, and Consolidation of Object Recognition Memory. The Journal of Neuroscience, 25(1), 52-61.

Yizhar, O., Fenno, Lief E., Davidson, Thomas J., Mogri, M., \& Deisseroth, K. (2011). Optogenetics in Neural Systems. Neuron, 71(1), 9-34. 
Chapter 5

General Discussion 
Hebbian-based models of memory are founded on the idea that memory retrieval requires the reactivation of neural circuits, or engrams, wired together during learning (Takeuchi, Duszkiewicz, \& Morris, 2014). However, until recently it has not been possible to determine whether retrieving a memory involves reactivation of the same neurons engaged during learning. Using a novel transgenic animal, the H2B-GFP TetTag mouse, we have demonstrated for the first time that neurons in the hippocampus and neocortex reactivate during the retrieval of context fear memory (Chapter Two). We next showed that patterns of retrieval-driven reactivation shift over time in the hippocampus and amygdala, while remaining stable in the cortex (Chapter Three). Finally, we demonstrated that although the hippocampus is required for neocortical reactivation several days after training, cortical regions may compensate when this region is inactivated following a weeks-long consolidation period (Chapter Four).

Chapter Two examined whether neurons engaged during contextual fear conditioning reactivate during subsequent memory retrieval. We first demonstrated that the H2B-GFP TetTag system can be used to selectivity and persistently tag neurons activated during learning. We observed that H2B-GFP expression in transgenic mice was increased by learning and did not occur when mice were administered doxycycline chow. Using this system, we found a large network of neurons in the hippocampus, amygdala and neocortex that were active during context fear conditioning and subsequent memory retrieval 2 days later. We next demonstrated that reactivation is contingent on memory retrieval, as we failed to observe greater than chance reactivation when animals were trained and tested in different environments. Reactivation was also decreased in a control 
condition where mice were trained but not tested. Finally, reactivation was not observed in the secondary motor cortex: a control brain region presumed not to directly support contextual memory retrieval. Together, these results confirmed our hypothesis that retrieving a recently formed context fear memory induces widespread reactivation of neurons in the hippocampus, amygdala, and neocortex.

Chapter Three examined whether reactivation continues to accompany memory retrieval when mice are tested at a longer time interval. We first established that inactivation of the dorsal hippocampus prior to context testing impaired memory retrieval when performed two days, but not two weeks after training. This suggested a) that the systems mediating contextual memory retrieval reorganize within two weeks of learning in H2B-GFP TetTag mice, and b) that we could use 14 days as a remote retrieval time point for this transgenic system. We next observed that neurons in the hippocampus (CA1 and CA3) and neocortex (ENTl and PTLp) reactivate in TetTag mice trained and tested 14 days later in the same environment. By directly comparing the percentage of H2B-GFP cells that reactivate two versus fourteen days after training, we determined that patterns of reactivation shift in the hippocampus and amygdala, but remain largely unchanged in the cortex over time. This result suggests that hippocampal and amygdala circuits are modified after learning, while cortical networks remain relatively stable.

In Chapter Four, we used the H2B-GFP TetTag system to address two fundamental assumptions of the Standard Model of Systems Consolidation (SMC): a) early after learning, the hippocampus drives reactivation of neocortical ensembles during memory 
retrieval, and b) following consolidation, neocortical ensembles reactivate independently (Alvarez \& Squire, 1994). To test these assumptions, we trained TetTag mice off doxycycline to label neocortical neurons. Hippocampus inactivation was then performed prior to memory retrieval two or fourteen days later. Hippocampus inactivation two days after training impaired memory retrieval and reduced c-Fos expression in CA1. Colocalization analysis revealed that $2 \mathrm{~d}$ inactivation also impaired neural reactivation in CA1 and reduced reactivation of neurons in the anterior cingulate and retrosplenial cortices. Hippocampus inactivation also eliminated patterns of correlated cortical activity that were observed in control animals during retrieval.

In contrast, inactivating the hippocampus fourteen days after training did not result in impaired freezing or significant decreases in cortical reactivation. When we performed structural equation modeling, we observed that inactivation at this later time point resulted unexpectedly in both an increase and decrease in coordinated cortical activity. This suggests that following a weeks-long consolidation period, cortical networks may be capable of reorganizing to compensate for hippocampus inactivation during retrieval: a finding supported by recent work by Deisseroth and colleagues (Goshen et al., 2011).

\section{Novel Techniques and Findings}

Prior to these experiments, fluorescence in situ hybridization (FISH) studies by Guzowski and colleagues demonstrated that hippocampal and neocortical neurons reactivate when animals explore the same spatial environment twice within a 30 minute period (Guzowski, McNaughton, Barnes, \& Worley, 1999; Kubik, Miyashita, Kubik-Zahorodna, 
\& Guzowski, 2012). This technique has also recently been used to show reactivation of lateral amygdala neurons following re-exposure to the same fear conditioned context across a 30-minute interval (Nomura, Nonaka, Imamura, Hashikawa, \& Matsuki, 2012). Longer retrieval intervals, however, cannot be investigated using this technique due to the rapid degradation of mRNA. In contrast, in-vivo recording methods are capable of following the activity of individual neurons across days and weeks. However, the potential for sparsely distributed learning circuits makes in-vivo recording an inappropriate tool for examining widespread retrieval-driven reactivation (Takeuchi et al., 2014).

To overcome these issues, we used novel TetTag mice developed by Mark Mayford at the Scripps Research Institute in La Jolla, CA. These animals express a long-lasting, activity-dependent form of GFP, human histone H2B-GFP: a fusion protein that takes several weeks to degrade (Kanda, Sullivan, \& Wahl, 1998). Using this system, we were able to tag neurons across the hippocampus, amygdala, and neocortex during learning and examine whether neurons reactivate when memory is retrieved days and weeks later. A similar tagging strategy was used by Mayford and colleagues to demonstrate the reactivation of amygdala neurons during associative memory retrieval three days after training in tau-LacZ reporter mice (Reijmers, Perkins, Matsuo, \& Mayford, 2007). Our experiments improve upon this study by examining reactivation across a collection of interconnected brain regions at both recent and remote time points. 
The experimental procedures used in this dissertation offer three methodological improvements for the study of retrieval-driven reactivation. First, we developed a novel quantification macro for use with Nikon NIS Elements software to aid in colocalization analysis (Nikon, Melville, NY). This macro increased the speed and ease of colocalization quantification by automatically detecting c-Fos and GFP positive cells based on their signal intensity, size, and circularity. Second, we developed a novel procedure for dorsal CA1 inactivation in which cannulae were inserted at a $20^{\circ}$ angle to avoid damage to overlying neocortical regions important for contextual memory retrieval (i.e. PTLp and RSPv). Finally, to ensure that c-Fos expression was induced by the retrieval test and not infusion handling we established a time course for peak c-Fos expression and degradation in CA1. A clearance time course for this immediate early gene was previously lacking in the literature.

Overall, the experiments outlined in this dissertation contribute five novel findings to the field of learning and memory: 1) neurons across the hippocampus, amygdala, and neocortex reactivate during memory retrieval. 2) Over time, reactivation patterns shift in the hippocampus and amygdala, while remaining more stable in the neocortex. 3) The level of CA1 activity during learning can be used to predict the strength of contextual memory retrieval occurring several days later. 4) The hippocampus supports both neocortical reactivation and intra-cortical coordination during recent memory retrieval. 5) Following a weeks-long consolidation period, neocortical regions are capable of shifting their patterns of functional connectivity to compensate for hippocampus inactivation and support continued memory retrieval. 


\section{Testing Assumptions of Current Models of Memory}

In this dissertation, we used the H2B-GFP TetTag system to test the accuracy of several fundamental assumptions made by the SMC. According to the Standard Model, episodic memories begin as patterns of activity within the hippocampus: a structure unique for its interconnectivity and integration of input from cortical association areas and extrahippocampal structures (Kandel, 2001; Rudy, 2008). Shortly after learning, the memory engram exists as a network of co-active hippocampal and neocortical neurons bound together through replay and NMDA-receptor dependent Hebbian plasticity (Kentros et al., 1998; Moscovitch, Nadel, Winocur, Gilboa, \& Rosenbaum, 2006; Takeuchi et al., 2014; Teyler \& DiScenna, 1986). Following a lengthy consolidation period, the development of new intra-cortical connections among learning-engaged neurons allows the cortex to support memory retrieval without input from the hippocampus (Alvarez \& Squire, 1994).

The SMC predicts, therefore, that damage to the hippocampus will affect newly formed memories, but spare older memories that have had time to consolidate within the cortex. This prediction was first challenged, however, by Nadel and Moscovitch's assertion that complete damage to the hippocampus results in the loss of both recent and remote episodic memories (Nadel \& Moscovitch, 1997). Nadel and Moscovitch proposed the Multiple Trace Theory (MTT) as an alternative to the Standard Model. According to this theory, the act of retrieving an episodic memory creates a new trace between the hippocampus and neocortex. Older episodic memories, which have been retrieved more 
often than new memories, appear at times to be resilient to hippocampus damage because of the redundancy of their hippocampus-based connections, not because they have achieved permanent storage within the cortex. In contrast to SMC, MTT predicts that hippocampus inactivation will still impair episodic memory retrieval and neocortical reactivation when performed following a consolidation period.

Although we cannot directly test episodic memory in mice, recent experiments demonstrate that rodents do form hippocampus-dependent "what-where-when" memories for specific experiences (Fellini \& Morellini, 2013). Many rodent studies have used hippocampus inactivation to demonstrate temporally graded retrograde amnesia and thus support the Standard Model (Frankland \& Bontempi, 2005; Kim \& Fanselow, 1992). However, a growing number of studies demonstrates the continued involvement of the hippocampus in spatial and contextual memory retrieval following a consolidation period (Goshen et al., 2011; Sutherland, Sparks, \& Lehmann, 2010; Wiltgen et al., 2010; Winocur, Moscovitch, \& Sekeres, 2013; Ziv et al., 2013). These recent findings support MTT and other alternative models to the SMC.

Therefore, in Chapter Four, we investigated whether hippocampus inactivation would a) impair memory retrieval and the reactivation of neocortical networks when performed two days after training, and b) fail to impair retrieval and cortical reactivation when performed several weeks later. We observed that the hippocampus is important for neocortical reactivation to occur during retrieval two days after training. This result supports both SMC and MTT (Alvarez \& Squire, 1994; Nadel \& Moscovitch, 1997; 
Winocur \& Moscovitch, 2011). In contrast, hippocampus inactivation two weeks after training did not impair memory retrieval or result in decreased cortical reactivation.

It is tempting to view these results as evidence for the Standard Model of Systems Consolidation. However, this interpretation may be inappropriate due to the lack of increased CNQX cortical reactivation from 2 to 14 days in our experiments. Second, if SMC was directly supported by our data, then we would expect to see 14-day hippocampus inactivation resulting in a sparing of cortical correlations observed in control animals. Instead, we observed both a numeric decrease in colocalization from 2 to 14 days and a lack of cortical correlations in the $14 \mathrm{~d}$ saline group. The emergence of $\mathrm{CA} 1 \rightarrow \mathrm{RSPv}$ and PTLp $\rightarrow$ RSPv coordination only occurred in CNQX animals following 14d hippocampus inactivation. Our results, therefore, may support a modified version of SMC where neocortical circuits are established after learning, but do not replace the original memory representations stored in the hippocampus (Winocur \& Moscovitch, 2011). As a result, hippocampus inactivation several weeks after training may induce the reorganization of cortical coordination in an attempt to compensate for the loss of hippocampus input.

The obvious drawback in Chapter Four was our inability to test predictions of SMC at a traditional 28-day interval (Frankland \& Bontempi, 2005; Squire, 1992; Wiltgen, Brown, Talton, \& Silva, 2004). This was due to the degradation of H2B-GFP signal in the amygdala and portions of the cortex after 14 days. However, future experiments described below can take advantage of recent advances in tagging and inactivation 
methodology to clarify the hippocampus' role in neocortical reactivation during remote memory retrieval.

\section{Future Directions}

Alternative tagging methods

Although the TetTag system is a powerful technique for labeling active neurons it has several limitations. First, gene tagging is dependent on the removal of doxycycline, which can take several days (Glazewski, Bejar, Mayford, \& Fox, 2001; Reijmers, Perkins, Matsuo, \& Mayford, 2007). Active neurons can be labeled anytime during this period, which results in elevated background expression relative to other methods (Guzowski, McNaughton, Barnes, \& Worley, 1999; Liu et al., 2012; Milanovic et al., 1998; Tayler, Tanaka, Reijmers, \& Wiltgen, 2013). Second, while H2B-GFP tagging is persistent, it is not permanent. The lack of a continuous feedback loop, like that present in Tau-LacZ reporter mice, results in the degradation of GFP signal several weeks after induction (between 14 and 28 days) (Reijmers et al., 2007). A recent study solved this problem by using a novel approach called TRAP: targeted recombination in active populations (Guenthner, Miyamichi, Yang, Heller, \& Luo, 2013). In these mice, activitydependent expression of a fluorescent effector gene is dependent on the presence of tamoxifen. The time window of activation is shorter in these mice (less than 12 hours) and background expression levels are very low. These mice could be extremely useful for long-term labeling studies because their activity-induced labeling is permanent.

A second limitation of the TetTag approach is that the percentage of reactivated 
hippocampal neurons is lower than that reported in catFISH studies. For example, $90 \%$ of labeled CA1 neurons reactivated when rats were placed in the same environment twice (Guzowski et al., 1999). In contrast, exposure to two identical environments in TetTag mice induces reactivation of only $20-40 \%$ of CA1 neurons (Nakazawa, Pevzer, \& Wiltgen, 2013; Tayler et al., 2013). There are many differences between these experiments (e.g. species, procedure, intervals between tests, background labeling) that could account for this difference. However, a recent study by Ziv et al. (2013) used optical imaging to examine place cell reactivation in mice and found that $\approx 25 \%$ of neurons in CA1 (with active place fields) were reactivated in the same environment 5 days later. This number decreased slightly over time to $\approx 15 \%$ when sessions were separated by 30 days. In this study, the authors used calcium imaging and a miniaturized head-mounted microscope to monitor neural activity as mice traversed a linear track. This method allowed them to examine thousands of neurons per mouse over a period of several weeks. The fact that reactivation levels in this study are similar to that observed in TetTag mice suggests that the percentage of reactivated neurons is smaller when tests are separated by several days as opposed to several minutes (as is the case in catFISH experiments).

\section{Optogenetic control of memory circuits}

A third limitation to the current experiments is the unspecific nature of pharmacological CA1 inactivation. Although our CNQX-induced freezing impairments were consistent across experiments, this method is not capable of specifically silencing only CA1 neurons that were previously active during learning. In future studies, optogenetic techniques 
could be combined with the TetTag or TRAP system to control the activity of previouslyactive cell populations via the expression of light-sensitive proteins such as channelrhodopsin (ChR2), halorhodopsin (NpHR), or archaerhodopsin (Arch) (Yizhar, Fenno, Davidson, Mogri, \& Deisseroth, 2011). The combination of optogenetics and a genetic, activity-dependent tagging system could be used to examine the contribution of previously active hippocampal neurons to neocortical reactivation during memory retrieval. For instance, activity-dependent expression of tTA could be used to drive the production of both a long-lasting tag and $\mathrm{ChR} 2$. According to recent experiments using a similar procedure by Tonegawa and colleagues, subsequent stimulation of tagged hippocampal neurons should induce memory retrieval (Liu et al., 2012). If current models of memory are correct, then optogenetic stimulation of hippocampal neurons should also lead to neocortical reactivation early after learning.

Unfortunately, optogenetic techniques are limited in their ability to recapitulate endogenous firing activity during light stimulation. Inducing artificial activity in the hippocampus could, therefore, alter the response of cortical neurons and limit reactivation. An alternative way to address this issue would be to inactivate tagged hippocampal neurons and determine the effects on neocortical reactivation. A recent study showed that NpHR can be used to silence hippocampal neurons and impair context fear memory retrieval (Goshen et al., 2011). In future studies, expression of this protein could be restricted to previously active neurons using methods similar to Liu et al. (2012). By silencing these neurons one could determine whether hippocampal activity is required for neocortical reactivation during recent, but not remote memory retrieval. 


\section{Concluding Remarks}

Recently developed neuroscience tools are making it possible to test basic assumptions of memory models for the first time. In this dissertation, we have used novel H2B-GFP TetTag animals to demonstrate that neural reactivation is an important component of both recent and remote memory retrieval. We have also demonstrated that the hippocampus is required for neocortical reactivation to occur several days after learning, but that cortical regions can reorganize their functional connectivity to compensate following consolidation. Future experiments testing the hippocampus' role in retrieval-driven reactivation will benefit from the use of optogenetics to achieve more specific control over the activity of neural networks. Using this method, researchers could determine whether the reactivation of previously-engaged hippocampal neurons drives the reactivation of neocortical circuits at recent and remote time points. 


\section{REFERENCES}

Alvarez, P., \& Squire, L. R. (1994). Memory consolidation and the medial temporal lobe: a simple network model. Proc Natl Acad Sci U S A, 91(15), 7041-7045.

Fellini, L., \& Morellini, F. (2013). Mice Create What-Where-When HippocampusDependent Memories of Unique Experiences. The Journal of Neuroscience, 33(3), 1038-1043.

Frankland, P. W., \& Bontempi, B. (2005). The organization of recent and remote memories. Nat Rev Neurosci, 6(2), 119-130.

Glazewski, S., Bejar, R., Mayford, M., \& Fox, K. (2001). The effect of autonomous alpha-CaMKII expression on sensory responses and experience-dependent plasticity in mouse barrel cortex. Neuropharmacology, 41(6), 771-778.

Goshen, I., Brodsky, M., Prakash, R., Wallace, J., Gradinaru, V., Ramakrishnan, C., \& Deisseroth, K. (2011). Dynamics of Retrieval Strategies for Remote Memories. Cell, 147(3), 678-689.

Guenthner, C. J., Miyamichi, K., Yang, H. H., Heller, H. C., \& Luo, L. (2013).

Permanent Genetic Access to Transiently Active Neurons via TRAP: Targeted Recombination in Active Populations. Neuron, 78(5), 773-784.

Guzowski, J. F., McNaughton, B. L., Barnes, C. A., \& Worley, P. F. (1999). Environment-specific expression of the immediate-early gene Arc in hippocampal neuronal ensembles. Nat Neurosci, 2(12), 1120-1124.

Kanda, T., Sullivan, K. F., \& Wahl, G. M. (1998). Histone-GFP fusion protein enables sensitive analysis of chromosome dynamics in living mammalian cells. Current Biology, 8(7), 377-385. 
Kandel, E. R. (2001). The Molecular Biology of Memory Storage: A Dialogue Between Genes and Synapses. Science, 294(5544), 1030-1038.

Kentros, C., Hargreaves, E., Hawkins, R. D., Kandel, E. R., Shapiro, M., \& Muller, R. V. (1998). Abolition of long-term stability of new hippocampal place cell maps by NMDA receptor blockade. Science, 280, 2121-2126.

Kim, J. J., \& Fanselow, M. S. (1992). Modality-specific retrograde amnesia of fear. Science, 256(5057), 675-677.

Kubik, S., Miyashita, T., Kubik-Zahorodna, A., \& Guzowski, J. (2012). Loss of activitydependent Arc gene expression in the retrosplenial cortex after hippocampal inactivation: interaction in a higher-order memory circuit. Neurobiology of Learning and Memory, Jan;97(1), 124-131.

Liu, X., Ramirez, S., Pang, P. T., Puryear, C. B., Govindarajan, A. F., Deisseroth, K., \& Tonegawa, S. (2012). Optogenetic stimulation of a hippocampal engram activates fear memory recall. Nature, 484(19 April ), 381-387.

Milanovic, S., Radulovic, J., Laban, O., Stiedl, O., Henn, F., \& Spiess, J. (1998).

Production of the Fos protein after contextual fear conditioning of C57BL/6N mice. Brain Res, 784(1-2), 37-47.

Moscovitch, M., Nadel, L., Winocur, G., Gilboa, A., \& Rosenbaum, R. S. (2006). The cognitive neuroscience of remote episodic, semantic and spatial memory. Current Opinion in Neurobiology, 16(2), 179-190.

Nadel, L., \& Moscovitch, M. (1997). Memory consolidation, retrograde amnesia and the hippocampal complex. Curr Opin Neurobiol, 7(2), 217-227. 
Nakazawa, Y., Pevzer, Z., \& Wiltgen, B. J. (2013). Stable reactivation of memory traces in the hippocampus and neocortex. Paper presented at the Society for Neuroscience Meeting, San Diego, CA.

Nomura, H., Nonaka, A., Imamura, N., Hashikawa, K., \& Matsuki, N. (2012). Memory coding in plastic neuronal subpopulations within the amygdala. NeuroImage, 60(1), 153-161.

Reijmers, L. G., Perkins, B. L., Matsuo, N., \& Mayford, M. (2007). Localization of a stable neural correlate of associative memory. Science, 317(5842), 1230-1233.

Rudy, J. W. (2008). The Neurobiology of Learning and Memory. Sunderland, MA: Sinauer Associates, Inc.

Squire, L. R. (1992). Memory and the hippocampus: A synthesis from findings with rats, monkeys, and humans. Psychological Review, 99(2), 195-231.

Sutherland, R. J., Sparks, F. T., \& Lehmann, H. (2010). Hippocampus and retrograde amnesia in the rat model: a modest proposal for the situation of systems consolidation. [Review]. Neuropsychologia, 48(8), 2357-2369.

Takeuchi, T., Duszkiewicz, A. J., \& Morris, R. G. M. (2014). The synaptic plasticity and memory hypothesis: encoding, storage and persistence. Philosophical Transactions of the Royal Society B: Biological Sciences, 369(1633).

Tayler, K. K., Tanaka, K. Z., Reijmers, L. G., \& Wiltgen, B. J. (2013). Reactivation of Neural Ensembles during the Retrieval of Recent and Remote Memory. Curr Biol, 23(2), 99-106.

Teyler, T. J., \& DiScenna, P. (1986). The hippocampal memory indexing theory. Behav Neurosci., Apr(100(2)), 147-154. 
Wiltgen, B. J., Brown, R. A., Talton, L. E., \& Silva, A. J. (2004). New circuits for old memories: the role of the neocortex in consolidation. Neuron, 44(1), 101-108.

Wiltgen, B. J., Zhou, M., Cai, Y., Balaji, J., Karlsson, M. G., Parivash, S. N., . . Silva, A. J. (2010). The hippocampus plays a selective role in the retrieval of detailed contextual memories. Curr Biol, 20(15), 1336-1344.

Winocur, G., \& Moscovitch, M. (2011). Memory transformation and systems consolidation. Journal of the International Neuropsychological Society, 17(5), 766-780.

Winocur, G., Moscovitch, M., \& Sekeres, M. (In Press). Factors affecting graded and ungraded memory loss following hippocampal lesions. Neurobiology of Learning and Memory, http://dx.doi.org/10.1016/j.nlm.2013.10.001

Yizhar, O., Fenno, Lief E., Davidson, Thomas J., Mogri, M., \& Deisseroth, K. (2011). Optogenetics in Neural Systems. Neuron, 71(1), 9-34.

Ziv, Y., Burns, L. D., Cocker, E. D., Hamel, E. O., Ghosh, K. K., Kitch, L. J., . . . Schnitzer, M. J. (2013). Long-term dynamics of CA1 hippocampal place codes. Nat Neurosci, 16(3), 264-266. 
